TRANSACTIONS OF THE

AMERICAN MATHEMATICAL SOCIETY

Volume 352, Number 8 , Pages 3429-3472

S 0002-9947(00)02392-8

Article electronically published on April 14, 2000

\title{
JONES INDEX THEORY BY HILBERT C*-BIMODULES AND K-THEORY
}

\author{
TSUYOSHI KAJIWARA AND YASUO WATATANI
}

Dedicated to the 60th birthday of Professor Takeshi Hirai

\begin{abstract}
In this paper we introduce the notion of Hilbert $\mathrm{C}^{*}$-bimodules, replacing the associativity condition of two-sided inner products in Rieffel's imprimitivity bimodules by a Pimsner-Popa type inequality. We prove Schur's Lemma and Frobenius reciprocity in this setting. We define minimality of Hilbert $\mathrm{C}^{*}$-bimodules and show that tensor products of minimal bimodules are also minimal. For an $A$ - $A$ bimodule which is compatible with a trace on a unital $\mathrm{C}^{*}$-algebra $A$, its dimension (square root of Jones index) depends only on its $K K$-class. Finally, we show that the dimension map transforms the Kasparov products in $K K(A, A)$ to the product of positive real numbers, and determine the subring of $K K(A, A)$ generated by the Hilbert $\mathrm{C}^{*}$-bimodules for a $\mathrm{C}^{*}$-algebra generated by Jones projections.
\end{abstract}

\section{INTRODUCTION}

Strong Morita equivalence for $\mathrm{C}^{*}$-algebras $A$ and $B$ was introduced by M. A. Rieffel (43, 44 ) by the existence of an imprimitivity bimodule $X$, which is a left Hilbert $A$-module as well as a right Hilbert $B$-module with full $\mathrm{C}^{*}$-algebra valued inner products ${ }_{A}\langle$,$\rangle and \langle,\rangle_{B}$ such that ${ }_{A}\langle x, y\rangle z=x\langle y, z\rangle_{B}$. If $\sigma$-unital $\mathrm{C}^{*}$-algebras $A$ and $B$ are strongly Morita equivalent, then $K_{*}(A)$ and $K_{*}(B)$ are isomorphic by the imprimitivity bimodule, and they are stably isomorphic by a result of L. Brown, P. Green and M. A. Rieffel ([2]).

The purpose of this paper is to study a relation between the index theory invented by V. F. R. Jones ([23]) and K-theory for $\mathrm{C}^{*}$-algebras (cf. [1]). There exists a Ktheoretical obstruction even for the inclusion of simple $\mathrm{C}^{*}$-algebras of index two. Jones introduced an index for a subfactor $N$ of a type $I I_{1}$ factor $M$ in terms of the coupling constant $\operatorname{dim}_{N} L^{2}(M)([23])$, which can be identified with the elements of $K_{0}(N)$. The index of a subfactor $N \subset M$ is analyzed by the bimodule ${ }_{N} M_{M}$. The important point to note here is that Jones index is also regarded as an element of the Kasparov group " $K K(N, N)$ " ([26],[15]). We studied the inclusion of $\mathrm{C}^{*}$-algebras, introducing the index for $\mathrm{C}^{*}$-subalgebras in 47 . In this paper, we study the $\mathrm{C}^{*}$ index theory from the viewpoint of bimodules. We introduce the notion of a Hilbert $A$ - $B$ bimodule ${ }_{A} X_{B}$ by replacing the associativity condition ${ }_{A}\langle x, y\rangle z=x\langle y, z\rangle_{B}$ in Rieffel's imprimitivity bimodule by Pimsner-Popa type inequalities [41]. We are

Received by the editors April 28, 1995 and, in revised form, March 18, 1998.

2000 Mathematics Subject Classification. Primary 46L08, 46L80.

Key words and phrases. Hilbert $\mathrm{C}^{*}$-bimodule, K-theory, Jones index, subfactor.

Partially supported by the Grants-in-aid for Scientific Research, The Ministery of Education.

(C)2000 American Mathematical Society 
forced to postulate that Hilbert $\mathrm{C}^{*}$-bimodules have two-sided inner products in order to make the category of them have the notion of conjugation, which plays an important role in subfactor theory. We remark that considering two-sided inner products corresponds to the orientation of tangles [24].

A Hilbert $\mathrm{C}^{*}$-bimodule $X={ }_{A} X_{B}$ of finite type (i.e. finite Jones index type) gives an element $[X]$ in the Kasparov group $K K(A, B)([26,[27])$. The conjugate Hilbert $\mathrm{C}^{*}$-bimodule $\bar{X}={ }_{B} \bar{X}_{A}$ gives an element $[\bar{X}]$ in $K K(B, A)$. We considered the canonical map $i_{*}: K_{0}(A) \rightarrow K_{0}(B)$ and the transfer map $T: K_{0}(B) \rightarrow K_{0}(A)$ (47]). These two maps $i_{*}$ and $T$ are realized by elements of the Kasparov group corresponding to the Hilbert $\mathrm{C}^{*}$-bimodules ${ }_{A} X_{B}$ and ${ }_{B} \bar{X}_{A}$. In particular, $X \otimes_{B} \bar{X}$ gives an element in $K K(A, A)$ which induces "a multiplication map by $[B: A]$ " up to evaluating by a trace. Motivated by these considerations, for a Hilbert $A$ - $A$ bimodule $Y$ we shall show that the Jones index $\operatorname{Ind}\left[{ }_{A} Y_{A}\right]$ depends only on its $K K$ class $[Y]$ in $K K(A, A)$ when ${ }_{A} Y_{A}$ is compatible with a trace on $A$. Furthermore, the 'statistical' dimension $d(X)$ (i.e. the square root of $\operatorname{Ind}\left[{ }_{A} X_{B}\right]$ ) is a character on the subring of $K K(A, A)$ generated by Hilbert $\mathrm{C}^{*}$-bimodules of finite type compatible with a trace. For this purpose, we shall develop the basic theory of Hilbert $\mathrm{C}^{*}$ bimodules from scratch.

The importance of the theory of bimodules in operator algebras was first recognized by A. Connes ([5]) in terms of correspondence. S. Popa ([4] ) developed the theory of correspondences, which contains the study of Jones index for bimodules. Later A. Ocneanu introduced the notion of paragroup in [37, 38] as an invariant of subfactor which is well described in terms of bimodules of type $I I_{1}$-factors. V. S. Sunder studied bimodules of $I I_{1}$-factors from the viewpoint of hypergroups ([46]). S. Yamagami ([51]) developed modular theory for bimodules for general factors, including type III factors. Y. Denizeau and J.-F. Havet $([8,9])$ studied the correspondence of finite index for general von Neumann algebras, and obtained additivity and multiplicativity of dimension and Frobenius reciprocity. They used the ordinary Hilbert space arguments, which are replaced by Hilbert $\mathrm{C}^{*}$-bimodule arguments in this paper. D. Evans and Y. Kawahigashi's recent work ([14]) also is related to bimodules.

On the other hand the connection between algebraic quantum field theory, studied by S. Doplicher, R. Haag and J. E. Roberts ([10, 11]) and Jones index theory ([23]) was discovered by R. Longo $([34$, 35]) concerning the (superselection) sectors. Sectors on type III factors correspond to bimodules, and have been proved very useful as in [4], 31], and [20].

A certain $\mathrm{C}^{*}$-category ([17]) of endomorphisms of the $\mathrm{C}^{*}$-algebra of local observables was analyzed and a new duality theory for compact groups was given by S. Doplicher and J. E. Roberts ([12]) using a kind of abstract category. Recently S. Yamagami developed the study of categorical structure behind the subfactor theory in [49], [50]. Following these arguments, we study the categorical structure for Hilbert $\mathrm{C}^{*}$-bimodules.

Several people have considered the relation with index for $\mathrm{C}^{*}$-algebras. $\mathrm{M}$. Izumi 21] gives an interesting construction on $\mathrm{C}^{*}$-subalgebras of a Cuntz algebra with non-integer index. V. Deaconu ([7) computes the index for certain inclusions of circle algebras constructing a path model. He also ([6]) studies branched coverings. K. Kodaka ([30]) shows that there is an endomorphism of $A_{\theta}$ with index 4. M. Khoshkam ([29]) considers the crossed product, and C. Peligrad and W. Szymanski [40] study saturated actions of Hopf algebras. 
We note that our notion of Hilbert $C^{*}$-bimodules of finite type is also suggested by the finite separable equivalence among rings due to L. Kadison ([24]).

We shall sketch the content of each section in this paper.

Section 1 contains the definitions and fundamental facts on Hilbert $\mathrm{C}^{*}$-bimodules of finite type. For a Hilbert $\mathrm{C}^{*}$-bimodule $X$ of finite type, we define the right index $\mathrm{r}-\operatorname{Ind}[X]$, the left index $1-\operatorname{Ind}[X]$ and the index $\operatorname{Ind}[X]$. Then $\operatorname{Ind}[X]$ measures the failure from an imprimitivity bimodule between two $\mathrm{C}^{*}$-algebras.

In section 2, we discuss Schur's Lemma and Frobenius reciprocity.

In section 3 , we define the minimality condition for Hilbert $\mathrm{C}^{*}$-bimodules of finite type. We prove that minimal bimodules are all semisimple, and that the dimension map is additive on minimal bimodules. Moreover, we prove that tensor products of minimal bimodules are also minimal.

In section 4, we discuss categorical arguments concerning Hilbert $\mathrm{C}^{*}$-bimodules of finite type. We show that the category of Hilbert $\mathrm{C}^{*}$-bimodule of finite type constitutes a $\mathrm{C}^{*}$-category in the sense of [17]. We show that the Frobenius reciprocity theorem holds in this setting, and the duality map ( $\epsilon$-structure) gives the correspondence of Frobenius reciprocity.

In section 5, we define an element in $K K(A, B)$ for a Hilbert $A$ - $B$ bimodule of finite type and define $K K$-indices in $K K(A, A)$ and $K K(B, B)$. We can write down the transfer map between K-groups, by the conjugate of Hilbert $C^{*}$-bimodules. We prove that the dimension $d(X)$ for Hilbert $A$ - $A$ bimodule of finite type which are compatible with a trace depends only on its class in $K K(A, A)$.

Recently two interesting papers on Hilbert $\mathrm{C}^{*}$-bimodules have been written: L. G. Brown, J. A. Mingo and N. T. Shen ([3]) and R. Exel ([15]), but their definition of Hilbert $A-B$ bimodule is different from ours. In fact their definition is suitable for their purpose, but too strong for studying index theory for $\mathrm{C}^{*}$-bimodules.

Finally, the authors express their thanks to S. Yamagami for a clear explanation and discussion of his recent work on categorical structure in subfactor theory. The authors would like to thank C. Phillips for pointing out some errors in an early version of the manuscript.

In this paper, we assume that all $\mathrm{C}^{*}$-algebras are unital, and in many places we assume that the centers of $\mathrm{C}^{*}$-algebras consist only of scalars. We assume $\mathrm{C}^{*}$-algebras are separable when we consider $K K$-groups. For a $\mathrm{C}^{*}$-algebra $A$, we denote by $Z(A)$ the center of $A$. For a conditional expectation $E$ from $B$ to $A$ of index finite type, we denote by $\operatorname{Ind}[E]$ the index of $E$ in $Z(B)([47])$, and if $\operatorname{Ind}[E]$ is a scalar, we denote by Index $E$ the positive scalar such that $\operatorname{Ind}[E]=(\operatorname{Index} E) I$.

\section{Hilbert $\mathrm{C}^{*}$-Bimodule of Finite type}

We first recall the definition of left Hilbert $\mathrm{C}^{*}$-module introduced by Paschke (39]), used by Rieffel ([43]) to describe Morita equivalence, and studied by Kasparov ([27]) for $K K$ theory. Let $B$ be a unital $\mathrm{C}^{*}$-algebra.

Definition 1.1. Let $X$ be a $\mathbf{C}$-vector space. $X$ is called a left pre-Hilbert $A$-module if $X$ satisfies the following conditions.

(1) $X$ is a left $A$-module.

(2) $X$ has an $A$-valued inner product ${ }_{A}\langle$,$\rangle , and they satisfy the following con-$ ditions:

(a) ${ }_{A}\langle$,$\rangle is left linear and right conjugate linear.$ 
(b) For each $a \in A$, the equalities ${ }_{A}\langle a x, y\rangle=a_{A}\langle x, y\rangle$ and ${ }_{A}\langle x, a y\rangle=$ ${ }_{A}\langle x, y\rangle a^{*}$ hold for all $x, y \in X$.

(c) ${ }_{A}\langle x, x\rangle \geq 0$ for each $x \in X$, and ${ }_{A}\langle x, x\rangle=0$ if and only if $x=0$.

(d) ${ }_{A}\langle x, y\rangle={ }_{A}\langle y, x\rangle^{*}$ for each $x, y \in X$.

Moreover, if $X$ satisfies the following, we call $X$ a left Hilbert A-module.

(3) $X$ is complete with respect to the norm ${ }_{A}\|x\|=\left(\left\|{ }_{A}\langle x, x\rangle\right\|\right)^{1 / 2}$.

Similarly, we recall the definition of right Hilbert $\mathrm{C}^{*}$-modules ([43]). Let B be a unital $\mathrm{C}^{*}$-algebra.

Definition 1.2. Let $X$ be a $\mathbf{C}$-vector space. $X$ is called a right pre-Hilbert $B$ module if $X$ satisfies the following conditions.

(1) $X$ is a right $B$-module.

(2) $X$ has a $B$-valued inner product $\langle,\rangle_{B}$, and they satisfy the following conditions:

(a) $\langle,\rangle_{B}$ is left conjugate linear and right linear.

(b) For each $b \in B$ the equalities $\langle x, y b\rangle_{B}=\langle x, y\rangle_{B} b$ and $\langle x b, y\rangle_{B}=b^{*}\langle x, y\rangle_{B}$ hold for all $x, y \in X$.

(c) $\langle x, x\rangle_{B} \geq 0$ for each $x \in X$, and $\langle x, x\rangle_{B}=0$ if and only if $x=0$.

(d) $\langle x, y\rangle_{B}=\langle y, x\rangle_{B}^{*}$ for each $x, y \in X$.

Moreover, if $X$ satisfies the following, we call $X$ a right Hilbert B-module.

(3) $X$ is complete with respect to the norm $\|x\|_{B}=\left(\langle x, x\rangle_{B}\right)^{1 / 2}$.

We write $X_{B}$ when $X$ is a right Hilbert $B$-module, and ${ }_{A} X$ when $X$ is a left Hilbert A-module. Let $X_{B}$ and $Y_{B}$ be right Hilbert $B$-moudules. We denote by $\mathbf{L}_{B}\left(X_{B}, Y_{B}\right)$ be the set of linear maps $T$ from $X$ to $Y$ which are bounded in the sense that $\left\|\langle T x, T x\rangle_{B}\right\| \leq K\left\|\langle x, x\rangle_{B}\right\|$ for some positive constant $K$ and have adjoints $T^{*}$ with respect to the $B$-inner products, i.e. $\langle T x, y\rangle_{B}=\left\langle x, T^{*} y\right\rangle_{B}$. We recall that if $T$ is everywhere defined and has an adjoint, then $T$ is necessarily a closed operator, and it is bounded by the closed graph theorem. We put $\mathbf{L}_{B}\left(X_{B}\right)=\mathbf{L}_{B}\left(X_{B}, X_{B}\right)$.

For $x, y \in X$, we put $\Theta_{x, y} z=x\langle y, z\rangle_{B}$ for each $z \in X$. We call these (right) rank one operators. We denote by $\mathbf{K}_{B}\left(X_{B}\right)$ the set of norm closures of linear combinations of (right) rank one operators.

We use the similar notation ${ }_{A} \mathbf{L}\left({ }_{A} X,{ }_{A} Y\right),{ }_{A} \mathbf{L}\left({ }_{A} X\right)$ and ${ }_{A} \mathbf{K}\left({ }_{A} X\right)$ for left Hilbert $A$-modules $X$ and $Y$. We denote by ${ }^{*} T$ the adjoint of $T \in{ }_{A} \mathbf{L}\left({ }_{A} X,{ }_{A} Y\right)$ with respect to left $A$-inner products. i.e. ${ }_{A}\langle T x, y\rangle={ }_{A}\left\langle x,{ }^{*} T y\right\rangle$. We put $\tilde{\Theta}_{x, y} z={ }_{A}\langle z, y\rangle x$, and call these (left) rank one operators.

Let $A$ and $B$ be unital $\mathrm{C}^{*}$-algebras. We define Hilbert $\mathrm{C}^{*}$-bimodules as follows.

Definition 1.3. Let $X$ be a $\mathbf{C}$-vector space. $X$ is called a Hilbert $A-B$ bimodule if $X$ satisfies the following conditions.

(1) $X$ is a left pre-Hilbert $A$-module and also right pre-Hilbert $B$-module. Moreover the $A$-action and the $B$-action commute with each other.

(2) There exist positive constants $C_{1}$ and $C_{2}$ such that

$$
C_{1}\left\|_{A}\langle x, x\rangle\right\|^{1 / 2} \leq\left\|\langle x, x\rangle_{B}\right\|^{1 / 2} \leq C_{2}\left\|_{A}\langle x, x\rangle\right\|^{1 / 2}
$$

for each $x \in X$.

(3) $X$ is complete with respect to the $A$-norm ${ }_{A}\|\cdot\|$ (equivalently, the $B$-norm $\left.\|\cdot\|_{B}\right)$. 
(4) For $a \in A$ and $b \in B$ we denote $\pi(a) x=a x, \rho(b) x=x b$ for $x \in X$. Then $\pi(A) \subset \mathbf{L}_{B}\left(X_{B}\right)$ and $\rho(B) \subset{ }_{A} \mathbf{L}\left({ }_{A} X\right)$. Moreover, $\pi$ is a ${ }^{*}$-representation of $A$ and $\rho$ is an anti-*-representation of $B$.

We write $X={ }_{A} X_{B}$ when we regard $X$ as a Hilbert $A$ - $B$ bimodule, and denote by ${ }_{A} \operatorname{Hom}_{B}\left({ }_{A} X_{B},{ }_{A} Y_{B}\right)$ the set of linear operators which commute with $A$ - $B$ actions without any norm-boundedness assumption.

Definition 1.4. Let $X$ be a left pre-Hilbert $A$-module. $X$ is called full if the closed linear span of $\left\{{ }_{A}\langle x, y\rangle ; x, y \in X\right\}$ is equal to $A$. We make similar definitions for right pre-Hilbert modules and Hilbert $\mathrm{C}^{*}$-bimodules.

Lemma 1.5. Let $X$ be a full left pre-Hilbert A-module. Then the left representation $\pi$ of $A$ on $X$ is injective. The similar statement holds for a right pre-Hilbert module.

Proof. We assume $a x=0$ for all $x$ in $X$. Then

$$
{ }_{A}\langle a x, a y\rangle=a_{A}\langle x, y\rangle a^{*}=0
$$

Since $X$ is left full, it follows that $a A a^{*}=\{0\}$. Then we have $a=0$.

If $A$ is unital, we may use the algebraic linear span instead of the closed linear span for the definition of fullness. We assume that all Hilbert $\mathrm{C}^{*}$-bimodule are full with respect to both sides if we do not specify otherwise.

We define the equivalence of Hilbert $\mathrm{C}^{*}$-bimodules.

Definition 1.6. Let ${ }_{A} X_{B}$ and ${ }_{A} Y_{B}$ be Hilbert $\mathrm{C}^{*}$-bimodules. Then ${ }_{A} X_{B}$ and ${ }_{A} Y_{B}$ are called similar if there exists an $A$ - $B$ bimodule isomorphism $T: X \rightarrow Y$ such that $T$ and $T^{-1}$ are bounded and $T$ has adjoints with respect to ${ }_{A}\langle$,$\rangle and \langle,\rangle_{B}$. We say that ${ }_{A} X_{B}$ and ${ }_{A} Y_{B}$ are unitarily equivalent, and write ${ }_{A} X_{B} \simeq{ }_{A} Y_{B}$, if there exists an $A$ - $B$ bimodule isomorphism $T: X \rightarrow Y$ that is unitary with respect to both inner products $A\langle$,$\rangle and \langle,\rangle_{B}$.

Example. Let $E$ be a conditional expectation from a unital $\mathrm{C}^{*}$-algebra $B$ to a unital $\mathrm{C}^{*}$-subalgebra $A$ such that for some positive constant $\lambda$ we have $E\left(b^{*} b\right) \geq$ $\lambda b^{*} b$ for every $b \in B$. We put $X=B$ and define $A$ and $B$ valued inner products as follows:

$$
{ }_{A}\langle x, y\rangle=E\left(x y^{*}\right), \quad\langle x, y\rangle_{B}=x^{*} y .
$$

Then $X$ is made into a Hilbert $A$ - $B$ bimodule.

Lemma 1.7. Let $X$ be a right Hilbert B-module. Then the following four statements are equivalent.

(1) $\mathbf{L}_{B}\left(X_{B}\right)=\mathbf{K}_{B}\left(X_{B}\right)$.

(2) $X_{B}$ is a finitely generated projective right B-module and $X_{B}$ is self dual.

(3) There exist two sets $\left\{u_{1}, \ldots, u_{n}\right\} \subset X$ and $\left\{u_{1}^{\prime}, \ldots, u_{n}^{\prime}\right\} \subset X$ such that $x=$ $\sum_{i=1}^{n} u_{i}\left\langle u_{i}^{\prime}, x\right\rangle_{B}$ for each $x \in X$.

(4) There exists a set $\left\{u_{1}, \ldots, u_{n}\right\} \subset X$ such that $x=\sum_{i=1}^{n} u_{i}\left\langle u_{i}, x\right\rangle_{B}$ for each $x \in X$.

Proof. The equivalence between (1) and (2) is given in [45. Since the rest of the proof of this lemma seems to be known, we omit it.

Lemma 1.8. Let $X_{B}$ be a right Hilbert B-module, and let $\mathbf{L}_{B}\left(X_{B}\right)=\mathbf{K}_{B}\left(X_{B}\right)$. Let $p \in \mathbf{L}_{B}\left(X_{B}\right)$ be an idempotent which is selfadjoint with respect to the B-inner product. Then $Y_{B}=p\left(X_{B}\right)$ also has the property $\mathbf{L}_{B}\left(Y_{B}\right)=\mathbf{K}_{B}\left(Y_{B}\right)$. 
Proof. It is clear that $Y_{B}$ is a right Hilbert $B$-module. Let $\left\{u_{1}, \ldots, u_{n}\right\}$ be a subset in $X$ as in Lemma 1.7. Then

$$
p x=\sum_{i=1}^{n} u_{i}\left\langle u_{i}, p x\right\rangle_{B} .
$$

Multiplying by $p$ from the left, we get

$$
p x=\sum_{i=1}^{n} p u_{i}\left\langle u_{i}, p x\right\rangle_{B}=\sum_{i=1}^{n} p u_{i}\left\langle p u_{i}, p x\right\rangle_{B} .
$$

We may take $\left\{p u_{1}, \ldots, p u_{n}\right\}$ as a subset of $p X$.

Let ${ }_{A} X_{B}$ be a Hilbert $C^{*}$-bimodule. We call a family $\left\{u_{1}, \ldots, u_{n}\right\} \subset X$ a right $B$-basis if $x=\sum_{i=1}^{n} u_{i}\left\langle u_{i}, x\right\rangle_{B}$ for every $x \in X$. Similarly, we call a family $\left\{v_{1}, \ldots, v_{m}\right\} \subset X$ a left $A$-basis if $x=\sum_{j=1}^{m} A\left\langle x, v_{j}\right\rangle v_{j}$ for every $x \in X$.

Definition 1.9. Let $X=X_{B}$ be a right Hilbert $B$-module. $X$ is said to be of finite type if $\mathbf{L}_{B}\left(X_{B}\right)=\mathbf{K}_{B}\left(X_{B}\right)$. Similarly, a left Hilbet $A$-module $X={ }_{A} X$ is said to be of finite type if ${ }_{A} \mathbf{L}\left({ }_{A} X\right)={ }_{A} \mathbf{K}\left({ }_{A} X\right)$. Let $X={ }_{A} X_{B}$ be a Hilbert $\mathrm{C}^{*}$-bimodule. Then $X$ is said to be of finite type if $X$ is of finite type in the sense of left and right Hilbert $\mathrm{C}^{*}$-modules.

Lemma 1.10. Let ${ }_{A} X_{B}$ and ${ }_{A} Y_{B}$ be Hilbert $C^{*}$-bimodules of finite type. Then any right B-module map $T: X_{B} \rightarrow Y_{B}$ is automatically a finite rank operator, and therefore $T$ is bounded with respect to $\|\cdot\|_{B}$ and $T$ has an adjoint $T^{*}$ with respect to $\langle,\rangle_{B}$. Similar properties hold for a left A-module map $S:{ }_{A} X \rightarrow{ }_{A} Y$

Proof. In fact, if $\left\{u_{1}, \ldots, u_{n}\right\}$ is a right B-basis, then, for $x \in X$,

$$
T(x)=T\left(\sum_{i=1}^{n} u_{i}\left\langle u_{i}, x\right\rangle_{B}\right)=\sum_{i=1}^{n} T\left(u_{i}\right)\left\langle u_{i}, x\right\rangle_{B}
$$

and $T^{*}$ is given by

$$
T^{*}(y)=\sum_{i=1}^{n} u_{i}\left\langle T\left(u_{i}\right), y\right\rangle_{B}
$$

By the above lemma, if $X$ and $Y$ are of finite type, then ${ }_{A} \operatorname{Hom}_{B}\left({ }_{A} X_{B},{ }_{A} Y_{B}\right)$ coincides with $\mathbf{L}_{B}\left(X_{B}, Y_{B}\right) \cap{ }_{A} \mathbf{L}\left({ }_{A} X,{ }_{A} Y\right)$. In the following we usually assume that Hilbert $\mathrm{C}^{*}$-bimodules are of finite type, and do not worry about the differences any more. In particular, we put ${ }_{A} \operatorname{End}_{B}\left({ }_{A} X_{B}\right)={ }_{A} \operatorname{Hom}_{B}\left({ }_{A} X_{B},{ }_{A} X_{B}\right)$. Then

$$
{ }_{A} \operatorname{End}_{B}\left({ }_{A} X_{B}\right)=\mathbf{K}_{B}\left(X_{B}\right) \cap{ }_{A} \mathbf{K}\left({ }_{A} X\right)=\mathbf{L}_{B}\left(X_{B}\right) \cap \pi(A)^{\prime}=\rho(B)^{\prime} \cap{ }_{A} \mathbf{L}\left({ }_{A} X\right) .
$$

This algebra corresponds to the relative commutant algebra in a von Neumann algebra setting. We should note that two adjoints with respect to two inner products for ${ }_{A} \operatorname{End}_{B}\left({ }_{A} X_{B}\right)$ do not coincide in general. But in many typical examples, to be given later, they do coincide, and ${ }_{A} \operatorname{End}_{B}\left({ }_{A} X_{B}\right)$ turns out to be considered as a $\mathrm{C}^{*}$-algebra.

Example. Let $E$ be a conditional expectation from $B$ to $A$ of index finite type [47]. Then $X={ }_{A} B_{B}$ given in the example after Definition 1.6 is a Hilbert $\mathrm{C}^{*}$-bimodule of finite type. 
We describe an example of a Hilbert $\mathrm{C}^{*}$-bimodule which does not come from the inclusion relation directly.

Example. Let $A$ and $B$ be two $\mathrm{C}^{*}$-subalgebras of a $\mathrm{C}^{*}$-algebra $C$, let $E$ be a conditional expectation from $C$ to $A$, and let $F$ be a conditional expectation from $C$ to $B$ ( $F$ and $E$ are of index finite type). We make $C$ into an $A$ - $B$ bimodule in a canonical way, and we give two inner products in the following way:

$$
{ }_{A}\langle x, y\rangle=E\left(x y^{*}\right) \quad\langle x, y\rangle_{B}=F\left(x^{*} y\right)
$$

Then ${ }_{A} C_{B}$ is a Hilbert $\mathrm{C}^{*}$-bimodule of finite type.

With respect to the topology in a Hilbert $\mathrm{C}^{*}$-bimodule, the following lemma seems to be useful.

Lemma 1.11. Let $B$ be a $C^{*}$-algebra, and let $X=X_{B}$ be a right pre-Hilbert $B$ module. If there exists a set $\left\{u_{1}, \ldots, u_{n}\right\} \subset X$ such that

$$
\forall x \in X \quad x=\sum_{i=1}^{n} u_{i}\left\langle u_{i}, x\right\rangle_{B},
$$

then $X$ is complete with respect to the norm $\|x\|_{B}=\left\|\langle x, x\rangle_{B}\right\|^{1 / 2}$. Moreover, there exist an integer $n$ and an orthogonal projection $p$ in $B \otimes M_{n}(\mathbf{C})$ such that $X_{B}$ is isomorphic to $\left(p B^{n}\right)_{B}$, where the orthogonal projection $p$ is given by $\left(\left\langle u_{i}, u_{j}\right\rangle_{B}\right)_{i, j}$.

Proof. We shall only sketch the proof. For $x \in X$ we define a map $\alpha$ from $x$ to $B^{n}$ as follows:

$$
\alpha(x)=\left(\left\langle u_{i}, x\right\rangle_{B}\right)_{i=1, \ldots, n} \in B^{n} .
$$

$B^{n}$ itself is a right Hilbert $B$-module with the $B$-inner product $\langle\xi, \eta\rangle_{B}=\sum_{i=1}^{n} \xi_{i}^{*} \eta_{i}$. Then $\alpha$ is a right $B$-module homomorphism, and conserves $B$-inner products and injectives.

We define $p \in B \otimes M_{n}$ by $p=\left(p_{i, j}\right)_{i, j}=\left(\left\langle u_{i}, u_{j}\right\rangle_{B}\right)_{i, j}$. Then $p$ is a self adjoint projection by the basis property of $\left\{u_{i}\right\}_{i=1, \ldots, n}$. Then, $p B^{n} \subset B^{n}$ is a right Hilbert $B$-submodule of $B^{n}$ and, in particular, $p B^{n}$ is complete.

Moreover we may show that $\alpha$ is an injective isomorphism from $X$ to $p B^{n}$.

Remark. If we define $\beta$ from $p B^{n}$ to $X$ by

$$
\beta(z)=\sum_{i=1}^{n} u_{i} z_{i}
$$

then $\beta=\alpha^{-1}$.

Example. Let $G=\mathbf{R}, K=\mathbf{Z}$ and $H=2 \pi \mathbf{Z}$. Let $\tilde{K}$ be a subgroup of $K$ and $\tilde{H}$ be a subgroup of $H$ with finite indices. Put $A=C(G / K) \rtimes \tilde{H}$ and $B=C(G / H) \rtimes \tilde{K}$. Then some completion $X$ of $C_{c}(G)$ of continuous functions of compact support is made into a Hilbert $\mathrm{C}^{*}$-bimodule of finite type as follows.

For $\tilde{h} \in \tilde{H}, \varphi \in C(G / K)$ and $x \in C_{c}(G)$, we put $(\tilde{h} \cdot x)(g)=x(-\tilde{h}+g)$, and $(\varphi \cdot x)(g)=\varphi(\dot{g}) x(g)$, where $\dot{g}$ means the image of $g$ in $G / K$. For $\tilde{h} \in \tilde{K}$, $\psi \in C(G / H)$ and $x \in C_{c}(G)$, we put $(x \cdot \tilde{k})(g)=x(g+\tilde{k})$ and $(x \cdot \psi)(g)=\psi(\dot{\dot{g}}) x(g)$, where $\dot{g}$ means the image of $g$ in $G / H$. We define a left $A$-action and a right $B$-action by the integrated form of the above covariant actions. 
Inner products are as follows:

$$
\begin{aligned}
& { }_{A}\langle x, y\rangle(\tilde{h}, \dot{g})=\sum_{k \in K} x(g+k) \overline{y(\tilde{h}-k-g)}, \\
& \langle x, y\rangle_{B}(\tilde{k}, \dot{g})=\sum_{h \in H} \overline{x(-g+h)} y(-h+g+\tilde{k}) .
\end{aligned}
$$

The next proposition is very useful.

Proposition 1.12. Let $X$ be a $\mathbf{C}$-vector space with a map ${ }_{A}\langle$,$\rangle from X \times X$ to $A$ and $a$ map $\langle,\rangle_{B}$ from $X \times X$ to $B$, and left $A$-action and right $B$-action which commute each other. We assume the following (a)-(d).

(a) ${ }_{A}\left\langle x+x^{\prime}, y\right\rangle={ }_{A}\langle x, y\rangle+{ }_{A}\left\langle x^{\prime}, y\right\rangle$ and ${ }_{A}\langle x, y\rangle^{*}={ }_{A}\langle y, x\rangle$ for $x, x^{\prime}$ and $y \in X$.

(b) $\left\langle x, y+y^{\prime}\right\rangle_{B}=\langle x, y\rangle_{B}+\left\langle x, y^{\prime}\right\rangle_{B}$ and $\langle x, y\rangle_{B}^{*}=\langle y, x\rangle_{B}$ for $x, y$ and $y^{\prime} \in X$.

(c) ${ }_{A}\langle a x, y\rangle=a_{A}\langle x, y\rangle$ for $a \in A$ and $x, y \in X$.

(d) $\langle x, y b\rangle_{B}=\langle x, y\rangle_{B} b$ for $b \in B$ and $x, y \in X$.

If $X$ has a family $\left\{u_{i}\right\}_{i=1, \ldots, n}$ such that $x=\sum_{i=1}^{n} u_{i}\left\langle u_{i}, x\right\rangle_{B}$ for every $x \in X$, then the following hold.

(1) $\langle x, x\rangle_{B} \geq 0$, and $\langle x, x\rangle_{B}=0$ if and only if $x=0$.

(2) $X$ is complete with respect to the norm $x \rightarrow\left\|\langle x, x\rangle_{B}\right\|^{1 / 2}$.

(3) $\langle a x, a x\rangle_{B} \leq\|a\|^{2}\langle x, x\rangle_{B}$ for $a \in A$ and $x \in X$.

If $X$ has a family $\left\{v_{j}\right\}_{j=1, \ldots, m}$ such that $x=\sum_{j}\left\langle\left\langle x, v_{j}\right\rangle v_{j}\right.$ for every $x \in X$, then statements similar to (1), (2) and (3) hold for ${ }_{A}\langle$,$\rangle .$

Proof. Let $x \in X$. Then we have

$$
\langle x, x\rangle_{B}=\left\langle\sum_{i=1}^{n} u_{i}\left\langle u_{i}, x\right\rangle_{B}, x\right\rangle_{B}=\sum_{i=1}^{n}\left\langle u_{i}, x\right\rangle_{B}^{*}\left\langle u_{i}, x\right\rangle_{B} .
$$

This shows that $\langle x, x\rangle_{B} \geq 0$, and $\langle x, x\rangle_{B}=0$ if and only if $x=0$. By the positivity of $\langle,\rangle_{B}$ the left representation of $A$ on $X$ is contractive. By Lemma 1.11, $X$ is complete.

This proposition shows that analytic properties follow from purely algebraic properties.

We define several concepts of indices for Hilbert $\mathrm{C}^{*}$-bimodules. Let ${ }_{A} X_{B}$ be a Hilbert $\mathrm{C}^{*}$-bimodule of finite type. We take a right $B$-basis $\left\{u_{1}, \ldots, u_{n}\right\}$ in $X$ and a left $A$-basis $\left\{v_{1}, \ldots, v_{m}\right\}$.

Proposition 1.13. The element $\sum_{i=1}^{n}{ }_{A}\left\langle u_{i}, u_{i}\right\rangle$ is independent of the choice of the right B-basis $\left\{u_{i}\right\}_{i}$, and $\sum_{i=1}^{m}\left\langle v_{i}, v_{i}\right\rangle_{B}$ is independent of the choice of the left $A$ basis $\left\{v_{j}\right\}_{j}$. Moreover, $\sum_{i=1}^{n} A\left\langle u_{i}, u_{i}\right\rangle$ is contained in $Z(A)$ and $\sum_{j=1}^{m}\left\langle v_{j}, v_{j}\right\rangle_{B}$ is contained in $Z(B)$.

Proof. We take another right $B$-basis $\left\{u_{1}^{\prime}, \ldots, u_{p}^{\prime}\right\}$. Then

$$
\begin{aligned}
\sum_{i=1}^{n}{ }_{A}\left\langle u_{i}, u_{i}\right\rangle & =\sum_{i=1}^{n}{ }_{A}\left\langle\sum_{k=1}^{p} u_{j}^{\prime}\left\langle u_{k}^{\prime}, u_{i}\right\rangle_{B}, u_{i}\right\rangle \\
& =\sum_{i, k}{ }_{A}\left\langle u_{k}^{\prime}, u_{i}\left\langle u_{i}, u_{k}^{\prime}\right\rangle_{B}\right\rangle=\sum_{k=1}^{p}{ }_{A}\left\langle u_{k}^{\prime}, u_{k}^{\prime}\right\rangle
\end{aligned}
$$

The other statement is similar. 
Definition 1.14. We put

$$
\mathrm{r}-\operatorname{Ind}[X]=\sum_{i=1}^{n}{ }_{A}\left\langle u_{i}, u_{i}\right\rangle \quad \mathrm{l}-\operatorname{Ind}[X]=\sum_{j=1}^{m}\left\langle v_{j}, v_{j}\right\rangle_{B}
$$

We call r-Ind $[X]$ the right index of ${ }_{A} X_{B}$ and l-Ind $[X]$ the left index of ${ }_{A} X_{B}$. When either $\mathrm{r}-\operatorname{Ind}[X]$ or $1-\operatorname{Ind}[X]$ is a scalar, we put $\operatorname{Ind}[X]=\mathrm{r}-\operatorname{Ind}[X] \cdot \ln \operatorname{Ind}[X]$, and call this the index of $X$.

Lemma 1.15. Let ${ }_{A} X_{B}$ be a Hilbert $C^{*}$-bimodule of finite type. For positive constants $C_{1}$ and $C_{2}$ we define ${ }_{A}\langle,\rangle^{\prime}$ and $\langle,\rangle_{B}^{\prime}$ by

$$
{ }_{A}\langle x, y\rangle^{\prime}=C_{1 A}\langle x, y\rangle, \quad\langle x, y\rangle_{B}^{\prime}=C_{2}\langle x, y\rangle_{B},
$$

where $x, y \in X$. Let $X^{\prime}$ be $X$ with these new inner products. Then

$$
\begin{aligned}
\operatorname{r-Ind}\left[X^{\prime}\right] & =\frac{C_{1}}{C_{2}}(\mathrm{r}-\operatorname{Ind}[X]), \\
\operatorname{l-Ind}\left[X^{\prime}\right] & =\frac{C_{2}}{C_{1}}(1-\operatorname{Ind}[X]), \\
\operatorname{Ind}\left[X^{\prime}\right] & =\operatorname{Ind}[X] .
\end{aligned}
$$

Proof. This is clear from the definition. We note that left and right basis changes follow the change of inner products.

Definition 1.16. We assume that $Z(A)=\mathbf{C} \cdot I, Z(B)=\mathbf{C} \cdot I$. Let $X={ }_{A} X_{B}$ be a Hilbert $A-B$ bimodule of finite type. Then $X$ is called normalized if $\mathrm{r}-\operatorname{Ind}(X)=$ $\operatorname{l-Ind}(X)$.

If $X$ is normalized, $\operatorname{Ind}[X]=(\operatorname{r}-\operatorname{Ind}[X])^{2}=(1-\operatorname{Ind}[X])^{2}$. We can always renormalize bimodules by Lemma 1.15 .

Let $X={ }_{A} X_{B}$ be a Hilbert $\mathrm{C}^{*}$-bimodule of finite type. Put $Y=X \otimes \mathbf{C}^{n}$. We may make $Y$ into an $A-B \otimes M_{n}(\mathbf{C})$ bimodule in a canonical way. We define a left $A$-inner product and a right $B \otimes M_{n}(\mathbf{C})$-inner product as follows. For $\tilde{x}=$ $\left(x_{1}, \ldots, x_{n}\right), \tilde{y}=\left(y_{1}, \ldots, y_{n}\right) \in Y$,

$$
{ }_{A}\langle\tilde{x}, \tilde{y}\rangle=\sum_{i=1}^{n}{ }_{A}\left\langle x_{i}, y_{i}\right\rangle, \quad\langle\tilde{x}, \tilde{y}\rangle_{B \otimes M_{n}(\mathbf{C})}=\left(\left\langle x_{i}, y_{j}\right\rangle_{B}\right)_{i, j}
$$

Then we need the following lemma.

Lemma 1.17. The above $Y$ is a Hilbert $A-B \otimes M_{n}(\mathbf{C})$ bimodule of finite type. Moreover, if $X$ has a right $B$-basis consisting of $n$ elements, then $B \otimes M_{n}(\mathbf{C})$ has a right $B$-basis consisting of only one element.

Proof. Let $\left\{u_{i}\right\}_{i}$ be a right $B$-basis in $X$. We take a canonical basis $\left\{e_{p}\right\}_{p}$ in $\mathbf{C}^{n}$. Then $\left\{u_{i} \otimes e_{p}\right\}_{i, p}$ is a right $B \otimes M_{n}(\mathbf{C})$-basis in $X \otimes \mathbf{C}^{n}$. Similarly, $\left\{v_{j} \otimes e_{i}\right\}_{j, p}$ is a left $A$-basis in $X \otimes \mathbf{C}^{n}$ for a left $A$-basis $\left\{v_{j}\right\}_{j}$ in $X$. The analytic properties follow by Proposition 1.12 . 
Now we assume that $X$ has a right $B$-basis $\left\{u_{1}, \ldots, u_{n}\right\}$. For, $\tilde{u}=\left(u_{1}, u_{2}, \ldots, u_{n}\right)$ and $\tilde{x}=\left(x_{1}, x_{2}, \ldots, x_{n}\right)$, we have

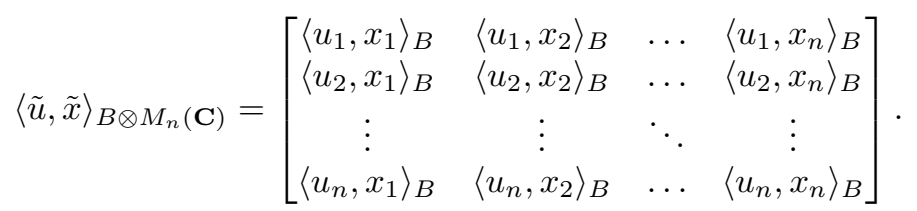

We have

$$
\begin{gathered}
\tilde{u}\langle\tilde{u}, \tilde{x}\rangle_{B \otimes M_{n}(\mathbf{C})}=\left(\sum_{i} u_{i}\left\langle u_{i}, x_{1}\right\rangle_{B}, \sum_{i} u_{i}\left\langle u_{i}, x_{2}\right\rangle_{B}, \ldots, \sum_{i} u_{i}\left\langle u_{i}, x_{n}\right\rangle_{B}\right) \\
=\left(x_{1}, x_{2}, \ldots, x_{n}\right)=\tilde{x} .
\end{gathered}
$$

Condition (2) in the definition of Hilbert $\mathrm{C}^{*}$-bimodule is automatically satisfied if it is of finite type. The next proposition is an analogue of the Pimsnor-Popa inequality.

Proposition 1.18. Let $A$ and $B$ be two $C^{*}$-algebras, let $X$ be a left Hilbert $A$ module and a right Hilbert $B$-module, and let $\pi$ be $a^{*}$ - representation of $A$ in $\mathbf{L}_{B}\left(X_{B}\right)$ and $\rho$ be $a^{*}$-representation of $B$ in ${ }_{A} \mathbf{L}\left({ }_{A} X\right)$. We assume that $X$ has a right $B$-basis $\left\{u_{1}, \ldots, u_{n}\right\}$. Then the following inequality holds:

$$
\left\|{ }_{A}\langle x, x\rangle\right\| \leq\left\|\sum_{i=1}^{n}{ }_{A}\left\langle u_{i}, u_{i}\right\rangle\right\|\left\|\langle x, x\rangle_{B}\right\| .
$$

Assume that $X$ has a left $A$-basis $\left\{v_{1}, \ldots, v_{m}\right\}$. Then

$$
\left\|\langle x, x\rangle_{B}\right\| \leq\left\|\sum_{j=1}^{m}\left\langle v_{j}, v_{j}\right\rangle_{B}\right\|\left\|_{A}\langle x, x\rangle\right\| .
$$

In particular, if $X$ has a left $A$-basis and a right $B$ basis, then all two-sided norms in $X$ are equivalent.

Proof. First we prove this theorem in the case that the basis consists of only one element. i.e. $x=u\langle u, x\rangle_{B}$. Then

$$
\langle x, x\rangle_{B}=\left\langle x, u\langle u, x\rangle_{B}\right\rangle_{B}=\langle u, x\rangle_{B}^{*}\langle u, x\rangle_{B} .
$$

We have $\left\|\langle x, x\rangle_{B}\right\|=\left\|\langle u, x\rangle_{B}\right\|^{2}$. On the other hand,

$$
{ }_{A}\langle x, x\rangle={ }_{A}\left\langle u\langle u, x\rangle_{B}, u\langle u, x\rangle_{B}\right\rangle \leq{ }_{A}\langle u, u\rangle\left\|\langle u, x\rangle_{B}\right\|^{2} .
$$

Using these, we have

$$
\left\|_{A}\langle x, x\rangle\right\| \leq\left\|{ }_{A}\langle u, u\rangle\right\| \cdot\left\|\langle x, x\rangle_{B}\right\| .
$$

We proceed to the general case. Let $Y=X \oplus X \oplus \cdots \oplus X=X \otimes \mathbf{C}^{n}$. Then $Y$ has been made into an $A-B \otimes M_{n}(\mathbf{C})$ bimodule. We put $\tilde{u}=\left(u_{1}, u_{2}, \ldots, u_{n}\right)$, and $\tilde{x}=\left(x_{1}, x_{2}, \ldots, x_{n}\right)$. By Lemma 1.17 , we have $\tilde{x}=\tilde{u}\langle\tilde{u}, \tilde{x}\rangle_{B \otimes M_{n}(\mathbf{C})}$. We apply the first part of the proof:

$$
\left\|{ }_{A}\langle\tilde{x}, \tilde{x}\rangle\right\| \leq\left\|{ }_{A}\langle\tilde{u}, \tilde{u}\rangle\right\| \cdot\left\|\langle\tilde{x}, \tilde{x}\rangle_{B \otimes M_{n}(\mathbf{C})}\right\| .
$$

We put $\tilde{x}=(x, 0, \ldots, 0)$. We have

$$
\left\|_{A}\langle x, x\rangle\right\| \leq\left\|\sum_{i}{ }_{A}\left\langle u_{i}, u_{i}\right\rangle\right\| \cdot\left\|\langle x, x\rangle_{B}\right\| .
$$


In the similar way, we have

$$
\left\|\langle x, x\rangle_{B}\right\| \leq\left\|\sum_{j}\left\langle v_{j}, v_{j}\right\rangle_{B}\right\| \cdot\left\|_{A}\langle x, x\rangle\right\| .
$$

Corollary $1.19(43])$. Let $A$ and $B$ be unital $C^{*}$-algebras, and let $X$ be an imprimitivity bimodule between $A$ and $B$. Then two-sided norms of $X$ are equal.

Proof. Let $\left\{u_{i}\right\}_{i}$ be a right $B$-basis in $X$. Then

$$
x=\sum_{i} u_{i}\left\langle u_{i}, x\right\rangle_{B}=\sum_{i}{ }_{A}\left\langle u_{i}, u_{i}\right\rangle x .
$$

This shows that $\mathrm{r}-\operatorname{Ind}[X]=I$. Similarly, $\mathrm{l}-\operatorname{Ind}[X]=I$. By Proposition 1.18 the conclusion holds.

Remark. Imprimitivity is characterized by the property $\mathrm{r}-\operatorname{Ind}[X]=I$ and $1-\operatorname{Ind}[X]$ $=I$ in Corollary 1.28, and the condition $\left\|_{A}\langle x, x\rangle\right\|=\left\|\langle x, x\rangle_{B}\right\|$ for each $x \in X$ is not sufficent for imprimitivity, as the following example shows.

Example. Let $A=B=\mathbf{C}$ and $X=\mathbf{C}^{n}$. We define $A-B$ action naturally and put ${ }_{A}\langle\tilde{x}, \tilde{y}\rangle=\sum_{i=1}^{n} x_{i} \bar{y}_{i}$ and $\langle\tilde{x}, \tilde{y}\rangle_{B}=\sum_{i=1}^{n} \bar{x}_{i} y_{i}$ for $\tilde{x}, \tilde{y} \in X$. Then $\left\|_{A}\langle\tilde{x}, \tilde{x}\rangle\right\|=$ $\left\|\langle\tilde{x}, \tilde{x}\rangle_{B}\right\|$, but $X$ is not an imprimitivity $A-B$ bimodule

Remark. By the above example, the constants in Proposition 1.18 are not best possible, in contrast to the original Pimsner-Popa inequality.

Let $X, Y$ be two Hilbert $A$-B bimodules. Then $X \oplus Y$ is also a Hilbert $A$-B bimodule in a canonical way. If $X$ and $Y$ are of finite type, $X \oplus Y$ is also of finite type.

Let $X$ be a Hilbert $A$ - $B$ bimodule and $p$ be an idempotent in $\operatorname{End}\left({ }_{A} X_{B}\right)$ which is self adjoint with respect to both inner products. Then $X$ is decomposed into the direct sum of $X p$ and $X(1-p)$ as a Hilbert $A-B$ bimodule. If $p$ is self adjoint only with respect to the $B$-inner product, we can decompose $X$ only as an $A$ - $B$ bimodule and a right Hilbert $B$-module.

Let $A, B$ and $C$ be unital $\mathrm{C}^{*}$-algebras, $X$ a Hilbert $A-B$ bimodule, and $Y$ a Hilbert $B-C$ bimodule. We denote by $X \odot Y$ the algebraic tensor product of $X$ and $Y$, and $x \odot y$ the tensor product of $x \in X$ and $y \in Y$.

Definition 1.20. (1) We define a left A-right $C$-bimodule structure in $X \odot Y$ as follows:

$\forall a \in A, \forall c \in C \quad a(x \odot y)=a x \odot y, \quad(x \odot y) c=x \odot y c \quad \forall x \in X y \in Y$.

(2) We define an $A$-inner product and a $C$-inner product on $X \odot Y$ by

$$
\begin{aligned}
& { }_{A}\left\langle x_{1} \odot y_{1}, x_{2} \odot y_{2}\right\rangle={ }_{A}\left\langle x_{1 B}\left\langle y_{1}, y_{2}\right\rangle, x_{2}\right\rangle, \\
& \left\langle x_{1} \odot y_{1}, x_{2} \odot y_{2}\right\rangle_{C}=\left\langle y_{1},\left\langle x_{1}, x_{2}\right\rangle_{B} y_{2}\right\rangle_{C},
\end{aligned}
$$

for all $x_{1}, x_{2} \in X$ and all $y_{1}, y_{2} \in Y$.

Lemma 1.21. (1) For $z, z^{\prime} \in X \odot Y, a \in A$ and $b \in B$ we have ${ }_{A}\left\langle a z, z^{\prime}\right\rangle=$ $a_{A}\left\langle z, z^{\prime}\right\rangle$ and $\left\langle z, z^{\prime} b\right\rangle_{B}=\left\langle z, z^{\prime}\right\rangle_{B} b$.

(2) For $z, z^{\prime} \in X \odot Y$ we have ${ }_{A}\left\langle z, z^{\prime}\right\rangle^{*}={ }_{A}\left\langle z^{\prime}, z\right\rangle$ and $\left\langle z, z^{\prime}\right\rangle_{B}^{*}=\left\langle z^{\prime}, z\right\rangle_{B}$.

(3) For $z, z^{\prime} \in X \odot Y, a \in A$ and $b \in B$ we have $\left\langle a z, z^{\prime}\right\rangle_{B}=\left\langle z, a^{*} z^{\prime}\right\rangle_{B}$ and ${ }_{A}\left\langle z b, z^{\prime}\right\rangle={ }_{A}\left\langle z, z^{\prime} b^{*}\right\rangle$. 
(4) For $x, x^{\prime} \in X, y, y^{\prime} \in Y$ and $b \in B$ we have

$$
{ }_{A}\left\langle x b \odot y-x \odot b y, x^{\prime} \odot y^{\prime}\right\rangle=0, \quad\left\langle x b \odot y-x \odot b y, x^{\prime} \odot y^{\prime}\right\rangle_{C}=0 .
$$

Proof. (1), (2) and (3) follow from the corresponding properties of ${ }_{A}\langle$,$\rangle and \langle,\rangle_{B}$.

(4) follows from the definition of inner products and the algebraic properties of $A\langle$,$\rangle and \langle,\rangle_{B}$.

Let $J$ be the linear span of $\{x b \odot y-x \odot b y: x, y \in X, b \in B\}$. Then $J$ is an $A-B$ invariant subspace of $X$. By the previous lemma, two inner products are determined modulo $J$. We let $X \otimes_{B} Y$ be the quotient of $X \odot Y$ by $J$, and we write $x \otimes y$ for the quotient image of $x \odot y$ under $J$.

Lemma 1.22. If ${ }_{A} X_{B}$ and ${ }_{B} Y_{C}$ are of finite type, then ${ }_{A}\left(X \otimes_{B} Y\right)_{C}$ has a left $A$-basis and a right $C$-basis.

Proof. Let $\left\{u_{1}, \ldots, u_{n}\right\}$ be a right $B$-basis in $X$ and $\left\{v_{1}, \ldots, v_{m}\right\}$ a right $C$-basis in $X$. Then

$$
x=\sum_{i=1}^{n} u_{i}\left\langle u_{i}, x\right\rangle_{B}, \quad y=\sum_{j=1}^{m} v_{j}\left\langle v_{j}, y\right\rangle_{C} .
$$

The following calculation shows that the set $\left\{u_{i} \otimes v_{j}: i=1, \ldots, n, j=1, \ldots, m\right\}$ forms a basis in $X \otimes_{B} Y$ :

$$
\begin{aligned}
\sum_{i=1}^{n} \sum_{j=1}^{m}\left(u_{i} \otimes v_{j}\right)\left\langle u_{i} \otimes v_{j}, x \otimes y\right\rangle_{C} & =\sum_{i=n}^{n} \sum_{j=1}^{m}\left(u_{i} \otimes v_{j}\right)\left\langle v_{j},\left\langle u_{i}, x\right\rangle_{B} y\right\rangle_{C} \\
& =\sum_{i=1}^{n} u_{i} \otimes\left(\sum_{j=1}^{m} v_{j}\left\langle v_{j},\left\langle u_{i}, x\right\rangle_{B}^{*} y\right\rangle_{C}\right) \\
& =\sum_{i=1}^{n} u_{i} \otimes\left\langle u_{i}, x\right\rangle_{B}^{*} y \\
& =\sum_{i=1}^{n} u_{i}\left\langle u_{i}, x\right\rangle_{B} \otimes y=x \otimes y
\end{aligned}
$$

Similarly, we have a left $A$-basis.

By these arguments, the following proposition follows.

Proposition 1.23. ${ }_{A}\left(X \otimes_{B} Y\right)_{C}$ is a Hilbert $C^{*}$-bimodule of finite type without completion.

Proof. We may conclude this by Proposition 1.12 .

Lemma 1.24. Let $X_{1}, X_{2}$ be Hilbert $A-B$ bimodules of finite type, and $Y$ a Hilbert $B-C$ bimodule of finite type. Then

$$
\left(X_{1} \oplus X_{2}\right) \otimes_{B} Y \simeq\left(X_{1} \otimes_{B} Y\right) \oplus\left(X_{2} \otimes_{B} Y\right) .
$$

This isomorphism holds in the sense of Hilbert $A-C$ bimodules.

In particular, the projection giving the direct sum decomposition of the right hand side is self adjoint with respect to the $A$-inner product and the $C$-inner product.

Proof. This is clear. 
Lemma 1.25. Let $X={ }_{A} X_{B}$ a full Hilbert $A-B$ bimodule of finite type and $Y=$ ${ }_{B} Y_{C}$ a full Hilbert $B-C$ bimodule of finite type. We put

$$
P=\mathbf{L}_{B}\left(X_{B}\right) \otimes I_{Y}, \quad Q=I_{X} \otimes{ }_{B} \mathbf{L}\left({ }_{B} Y\right) .
$$

Then $X \otimes_{B} Y$ is a Hilbert $P$ - $Q$ bimodule, and we have

$$
\mathbf{L}_{Q}\left(\left(X \otimes_{B} Y\right)_{Q}\right)=P, \quad{ }_{P} \mathbf{L}\left({ }_{P}\left(X \otimes_{B} Y\right)\right)=Q .
$$

Proof. By definition, $\mathbf{L}_{B}(X) X_{B}$ and ${ }_{B} Y_{B} \mathbf{L}(Y)$ are imprimitivity bimodules. We see that ${ }_{P}\left(X \otimes_{B} Y\right)_{Q}$ is also an imprimitivity bimodule by checking the associativity condition for the inner products.

We must show that a Hilbert $\mathrm{C}^{*}$-bimodule of finite type can be decomposed into finitely many irreducible components.

Lemma 1.26. Let ${ }_{A} X_{B}$ be a Hilbert $C^{*}$-bimodule of finite type which is full. Then, there exists a conditional expectation $E$ from $\mathbf{L}_{B}\left(X_{B}\right)$ to $A$ such that $E$ is of index finite type. Moreover, if $\mathrm{r}-\operatorname{Ind}[X]$ or $1-\operatorname{Ind}[X]$ is a scalar, then $\operatorname{Ind}[E]=\operatorname{Ind}\left[{ }_{A} X_{B}\right]$ and

$$
E\left(\Theta_{x_{1}, x_{2}}\right)=(\mathrm{r}-\operatorname{Ind}[X])^{-1}\left\langle x_{1}, x_{2}\right\rangle .
$$

Proof. We take a right $B$-basis $\left\{u_{i}\right\}_{i=1, \ldots, n}$ in $X$. For $T \in \mathbf{L}_{B}\left(X_{B}\right)$, we put $F(T)=$ $\sum_{i=1}^{n} A\left\langle T u_{i}, u_{i}\right\rangle$. Then we can show that the right hand side is independent of the choice of left $A$-basis $\left\{u_{i}\right\}_{i}$.

Moreover, the following condition holds. For $T=\Theta_{x, y}$, we have $F(T)={ }_{A}\langle x, y\rangle$, and hence $F\left(a T a^{\prime}\right)=a F(T) a^{\prime}$. Then, $F$ is a bounded operator valued weight from $\mathbf{L}_{B}\left(X_{B}\right)$ to $A$.

We show that $F(I)$ is positive and invertible and is contained in $Z(A)$. Since the linear span of an $A$-inner product is surjective, there exist elements $x_{1}, \ldots, x_{n}$, $y_{1}, \ldots, y_{n} \in X_{B}$ such that $\sum_{i=1}^{n} A\left\langle x_{i}, y_{i}\right\rangle=I$. As in the proof of Lemma 2.1.6 in [47], we may assume that $x_{i}=y_{i}$ for $i=1, \ldots, n$. Since $\sum_{i=1}^{n} \Theta_{x_{i}, x_{i}}$ is selfadjoint and positive, there exists a positive constant $C$ such that $C \cdot I \geq \sum_{i=1}^{n} \Theta_{x_{i}, x_{i}}$. Since $I=\sum_{i=1}^{n} \Theta_{u_{i}, u_{i}}$,

$$
F(C \cdot I) \geq \sum_{i=1}^{n} F\left(\Theta_{x_{i}, x_{i}}\right)=\sum_{i=1}^{n}{ }_{A}\left\langle x_{i}, x_{i}\right\rangle
$$

This shows that

$$
\sum_{i=1}^{n}{ }_{A}\left\langle u_{i}, u_{i}\right\rangle \geq \frac{1}{C} \sum_{i=1}^{n}{ }_{A}\left\langle x_{i}, x_{i}\right\rangle=\frac{1}{C} I
$$

and that the left hand side is invertible.

We put

$$
E(T)=F(I)^{-1 / 2} F(T) F(I)^{-1 / 2} .
$$


Let $\left\{u_{i}\right\}_{i=1, \ldots, m}$ be a right $B$-basis and $\left\{v_{j}\right\}_{j=1, \ldots, n}$ a left $A$-basis. We show that $\left\{\Theta_{u_{i}, v_{j}}\right\}_{i=1, \ldots, m, j=1, \ldots, n}$ forms a quasi basis for $F$ in $\mathbf{L}_{B}\left(X_{B}\right)$ :

$$
\begin{aligned}
\sum_{i=1}^{m} \sum_{j=1}^{n} \Theta_{u_{i}, v_{j}} F\left(\Theta_{u_{i}, v_{j}}^{*} \Theta_{p, q}\right) & =\sum_{i=1}^{m} \sum_{j=1}^{n} \Theta_{u_{i}, v_{j} A}\left\langle v_{j}\left\langle u_{i}, p\right\rangle_{B}, q\right\rangle \\
& =\sum_{i=1}^{m} \sum_{j=1}^{n} \Theta_{u_{i, A}\left\langle q, v_{j}\left\langle u_{i}, p\right\rangle_{B}\right\rangle v_{j}} \\
& =\sum_{i=1}^{m} \sum_{j=1}^{n} \Theta_{u_{i}, A}\left\langle q\left\langle p, u_{i}\right\rangle_{B}, v_{j}\right\rangle v_{j} \\
& =\sum_{i=1}^{m} \Theta_{u_{i},\left\langle p, u_{i}\right\rangle_{B} q} \\
& =\Theta_{p, q} .
\end{aligned}
$$

We put $U_{i, j}=\Theta_{u_{i}, v_{j}} F(I)^{1 / 2}$. Then, since $F(I) \in Z(A)$,

$$
\begin{aligned}
\sum_{i=1, j=1}^{m, n} U_{i, j} E\left(U_{i, j}^{*} T\right) & =\sum_{i=1, j=1}^{m, n} \Theta_{u_{i}, v_{j}} F(I)^{1 / 2} F(I)^{-1 / 2} F\left(F(I)^{1 / 2} \Theta_{v_{j}, u_{i}} T\right) F(I)^{-1 / 2} \\
& =\sum_{i=1, j=1}^{m, n} \Theta_{u_{i}, v_{j}} F\left(\Theta_{v_{j}, u_{i}} T\right) \\
& =T .
\end{aligned}
$$

The family $\left\{U_{i, j}\right\}_{i=1, \ldots, m, j=1, \ldots, n}$ constitutes a quasi basis for the conditional expectation $E$, and this shows that $E$ is of finite index type.

We assume that $\mathrm{r}-\operatorname{Ind}[X]$ is a scalar. Then $F(I)=\mathrm{r}-\operatorname{Ind}[X]$ is also a scalar. Then

$$
\begin{aligned}
\operatorname{Ind}\left[{ }_{A} X_{B}\right] & =(\mathrm{r}-\operatorname{Ind}[X])\left(\sum_{i} \Theta_{u_{i}, u_{i}}\right)(\mathrm{l}-\operatorname{Ind}[X]) \\
& =\sum_{p}{ }_{A}\left\langle u_{p}, u_{p}\right\rangle \sum_{i, j} \Theta_{u_{i}\left\langle v_{j}, v_{j}\right\rangle_{B}, u_{i}} \\
& =F(I) \sum_{i, j} \Theta_{u_{i}\left\langle v_{j}, v_{j}\right\rangle_{B}, u_{i}} \\
& =\sum_{i, j} \Theta_{u_{i}, v_{j}} F(I)^{1 / 2} F(I)^{1 / 2} \Theta_{v_{j}, u_{i}} \\
& =\operatorname{Ind}[E] .
\end{aligned}
$$

Theorem 1.27. Let $X={ }_{A} X_{B}$ be a Hilbert $C^{*}$-bimodule of finite type. If the center of either $A$ or $B$ is scalar, ${ }_{A} \operatorname{End}_{B}\left({ }_{A} X_{B}\right)$ is of finite dimension.

Proof. We assume that the center of $A$ is scalar. The non-*-algebra ${ }_{A} \operatorname{End}_{B}\left({ }_{A} X_{B}\right)$ is identical to $A^{\prime} \cap \mathbf{L}_{B}\left(X_{B}\right)$. By Lemma 1.26, there exists a conditional expectation $E$ from $\mathbf{L}_{B}\left(X_{B}\right)$ to $A$ which is of index finite type. By Lemma 2.7.3 of [47, the dimension of $A^{\prime} \cap \mathbf{L}_{B}\left(X_{B}\right)$ is finite.

We may also show the characterization of imprimitivity bimodules for unital $\mathrm{C}^{*}$-algebras using Proposition 1.26. 
Corollary 1.28. Let $X={ }_{A} X_{B}$ be a Hilbert $C^{*}$-bimodule of finite type. Then $X$ is an imprimitivity bimodule if and only if $\mathrm{r}-\operatorname{Ind}[X]=I$ and $1-\operatorname{Ind}[Y]=I$.

Proof. Suppose that ${ }_{A} X_{B}$ is a imprimitivity bimodule. Let $\left\{u_{i}\right\}_{i=1, \ldots, n}$ be a right $B$-basis in $X$. Then for every $x \in X$,

$$
x=\sum_{i}^{n} u_{i}\left\langle u_{i}, x\right\rangle_{B}=\sum_{i}^{n}{ }_{A}\left\langle u_{i}, u_{i}\right\rangle x .
$$

Then we have r-Ind $[X]=\sum_{i=1}^{n} A\left\langle u_{i}, u_{i}\right\rangle=I$. Similarly, we have l-Ind $[X]=I$.

Conversely, we assume that $\mathrm{r}-\operatorname{Ind}[X]=I$ and l-Ind $[X]=I$. Then by Lemma 1.26 the inclusion $A \subset \mathbf{L}_{B}\left(X_{B}\right)$ of $\mathrm{C}^{*}$-algebras has index $I$ for the canonical conditional expectation. Then $A=\mathbf{L}_{B}\left(X_{B}\right)$. We must show that the associativity condition of inner products holds. $X$ has two left $A$-inner products, the original one and the other $A\left\langle, \gamma\right.$ given by the relation $A=\mathbf{L}_{B}\left(X_{B}\right)$. Then by Proposition 2.3 in chapter 2, there exists a $T \in{ }_{A} \operatorname{End}_{B}(X)=Z(A)$ such that ${ }_{A}\langle x, y\rangle^{r}={ }_{A}\langle T x, y\rangle$. By the definition $\sum_{i=1}^{n}{ }_{A}\left\langle u_{i}, u_{i}\right\rangle^{\sim}=I$, and by the assumption $\sum_{i=1}^{n}{ }_{A}\left\langle u_{i}, u_{i}\right\rangle=I$,

$$
I=\sum_{i=1}^{n}\left\langle u_{i}, u_{i}\right\rangle=\sum_{i=1}^{n}{ }_{A}\left\langle T u_{i}, u_{i}\right\rangle=T \sum_{i=1}^{n}{ }_{A}\left\langle u_{i}, u_{i}\right\rangle=T .
$$

This shows that $T=I$, and ${ }_{A} X_{B}$ is an imprimitivity bimodule between $A$ and $B$.

We define the conjugate of bimodules. Let $\bar{X}$ be $X$ itself when it is considered as a set. We write $\bar{x}$ when $x$ is considered in $\bar{X}$. $\bar{X}$ is made into a Hilbert $B-A$ bimodule as follows:

(1) $\bar{x}+\bar{y}=\overline{x+y}$ and $\lambda \bar{x}=\overline{(\bar{\lambda} x)}$ for all $x, y \in X, \lambda \in \mathbf{C}$.

(2) $b \cdot \bar{x} \cdot a=\overline{a^{*}} x b^{*}$ for all $a \in A, b \in B, x \in X$.

(3) ${ }_{B}\langle\bar{x}, \bar{y}\rangle=\langle x, y\rangle_{B}$ and $\langle\bar{x}, \bar{y}\rangle_{A}={ }_{A}\langle x, y\rangle$ for all $x, y \in X$.

Let ${ }_{A} X_{B}$ and ${ }_{A} Y_{B}$ be Hilbert $\mathrm{C}^{*}$-bimodules of finite type. We define the conjugate and transposes of morphisms. For $T \in \operatorname{Hom}\left({ }_{A} X_{B},{ }_{A} Y_{B}\right)$, we put $\bar{T} \bar{x}=\overline{T x}$. Then $\bar{T}$ is contained in $\operatorname{Hom}\left({ }_{B} \bar{X}_{A},{ }_{B} \bar{Y}_{A}\right)$. We call this the conjugate morphism of $T$. Moreover we put ${ }^{t} T={ }^{*} T, T^{t}=\overline{T^{*}}$, and call these the left transpose of $T$ and the right transpose of $T$.

We define duals of $A$ - $B$ Hilbert $\mathrm{C}^{*}$-modules. We define the right dual $X^{*}$ of $X$ by $\mathbf{L}_{B}\left(X_{B}, B_{B}\right)$, and the left dual ${ }^{*} X$ of $X$ by ${ }_{A} \mathbf{L}\left({ }_{A} X,{ }_{A} A\right) . X^{*}$ and ${ }^{*} X$ are Hilbert $B$ - $A$ bimodules. When $X$ is of finite type, $X$ is self dual as a left $A$-module and as a right $B$-module. Since the left dual module ${ }^{*} X$ and the right dual module $X^{*}$ are canonically isomorphic to the conjugate module $\bar{X}$ of $X$, we usually use the conjugate bimodule ${ }_{B} \bar{X}_{A}$. We write $x^{\vee}$ for $x$ in $\mathbf{L}_{B}\left(X_{B}, B_{B}\right),{ }^{\vee} x$ for $x$ in ${ }_{A} \mathbf{L}\left({ }_{A} X,{ }_{A} A\right)$.

Example. In the situation of the example after Definition $1.6 X^{*}={ }_{B} B_{A}$, and ${ }_{B}\left\langle x^{\vee}, y^{\vee}\right\rangle=x y^{*},\left\langle x^{\vee}, y^{\vee}\right\rangle_{A}=E\left(x^{*} y\right)$ for each $x, y \in X . x^{\vee}$ is really $x^{*}$ considered in a $\mathrm{C}^{*}$-algebra $B$. The correspondence $x^{\vee} \rightarrow \bar{x}$ gives the isomorphism between $X^{*}$ and $\bar{X}$.

We explain a family of examples of Hilbert $\mathrm{C}^{*}$-bimodules which do not come from inclusion relations, directly following the bundle construction in the von Neumann algebra case ([33]). 
Let $G$ be a countable discrete group, and let $H$ and $K$ be finite subgroups of $G$. Then a finite dimensional Hilbert space $V={ }_{H} V_{K}$ is called an $H$-K bundle over $G$ if $V=\bigoplus_{g \in G} V_{g}$, where the $V_{g}$ 's are finite dimensional Hilbert spaces, and there exist two commuting actions of $H$ and $K$ on $V$ which are compatible with the left translation of $H$ on $G$ and the right translation of $K$ on $G$, and conserve inner products on the $V_{g}$ 's. We admit the possibility $V_{g}=0$, and put $\operatorname{supp}(V)=\left\{g \in G: V_{g} \neq\{0\}\right\}$ and call this the support of $V$ ([33]). Supports of bundles are unions of $H$-K double cosets in $G$. We remark that the supports of $H-K$ bundles are all finite unions of $H-K$ double cosets.

Let $A$ be a unital $\mathrm{C}^{*}$-algebra and $\alpha$ an action of $G$ on $A$. For an $H-K$ bundle $V$ over $G$, we put

$$
\hat{V}=A \otimes{ }_{H} V_{K}=\oplus_{g \in G}\left(A \otimes V_{g}\right) .
$$

Tensor products mean algebraic tensor products: For each $g \in G, A \otimes V_{g}$ has two $A$-valued inner products:

$$
{ }_{A}\left\langle a \otimes v, a^{\prime} \otimes v^{\prime}\right\rangle=a a^{\prime *}\left\langle v, v^{\prime}\right\rangle, \quad\left\langle a \otimes v, a^{\prime} \otimes v^{\prime}\right\rangle_{A}=a^{*} a^{\prime}\left\langle v, v^{\prime}\right\rangle .
$$

We may construct two $\mathrm{C}^{*}$-crossed products, $P=A \rtimes_{\alpha} H$ and $Q=A \rtimes_{\alpha} K$. We make $\hat{V}$ into a Hilbert $P-Q$ bimodule.

For $x, y \in \hat{V}$, we define two inner products as follows:

$$
\begin{aligned}
& { }_{P}\langle x, y\rangle(h)=\sum_{g \in G} A\left\langle x(g),\left(\alpha_{h} \otimes h\right)\left(y\left(h^{-1} g\right)\right)\right\rangle, \\
& \left.\langle x, y\rangle_{Q}(k)=\sum_{g \in G} \alpha_{g}\left(\left\langle x\left(g^{-1}\right), y\left(g^{-1} k\right) \cdot k^{-1}\right)\right\rangle_{A}\right) .
\end{aligned}
$$

We define left $P$ action and right $Q$ action by the integrated form of the covariant actions as follows. Let $h \in H, k \in K$ and $a \in A$; then

$$
\begin{aligned}
& (h \cdot x)(g)=\left(\alpha_{h} \otimes h\right)\left(x\left(h^{-1} g\right)\right), \quad(a \cdot x)(g)=a x(g), \quad g \in G, \\
& (x \cdot k)(g)=x\left(g k^{-1}\right) \cdot k, \quad(x \cdot a)(g)=x(g) \alpha_{g}(a) .
\end{aligned}
$$

Let $n$ be the number of $H$-orbits in $\operatorname{supp}(V)$ and $m$ the number of $K$ orbits in $\operatorname{supp}(V)$.

Proposition 1.29. $\hat{V}$ is a Hilbert $C^{*}$-bimodule of finite type. If the support of $V$ consists of only one $H-K$ double coset, then l-Ind $[\hat{V}]=|V| n, \mathrm{r}-\operatorname{Ind}[\hat{V}]=|V| m$ and $\operatorname{Ind}[\hat{V}]=|V|^{2} m n$, where $|V|$ is the common dimension of a fiber $V_{g}$ for $g \in \operatorname{supp}(V)$.

Proof. The conclusions of Proposition 1.12 are verified by calculations except for fullness. We construct a left $P$-basis. Let $\Sigma$ be the support of $V$, and take a representative set $\left\{\sigma_{1}, \ldots, \sigma_{n}\right\}$ of the left $H$-coset space of $\Sigma$. We define functions $d_{i}$ on $\Sigma$, by $d_{i}(g)=1$ if $g=\sigma_{i}$ and 0 otherwise. Let $p_{i}$ be the dimension of $V_{\sigma_{i}}$. We take orthonormal bases $\left\{v_{1}^{i}, \ldots, v_{p_{i}}^{i}\right\}$ for every $V_{\sigma_{i}}$ and define orthonormal bases $\left\{v_{1}^{g}, \ldots, v_{p_{i}}^{g}\right\}$ of every $V_{g}$ for $g \in \operatorname{supp}(V)$ by left translation of $\left\{v_{1}, \ldots, v_{p_{i}}\right\}$ under $h$ if $g=h \cdot \sigma_{i}$ for some $i$. We put $u_{i, j}(g)=d_{i}(g) v_{j}^{g} \in V_{g}$. Then we may show that 
$\left\{u_{i, j}\right\}_{1 \leq i \leq n, 1 \leq j \leq p_{i}}$ is a left $H$ basis in $\hat{V}$ :

$$
\begin{aligned}
{ }_{P}\left\langle x, u_{i, j}\right\rangle u_{i, j}(g) & =\sum_{h \in H} P\left\langle x, u_{i, j}\right\rangle(h)\left(\alpha_{h} \otimes h\right)\left(u_{i, j}\left(h^{-1} g\right)\right) \\
& =\sum_{h \in H, g^{\prime} \in \Sigma}\left\langle x\left(g^{\prime}\right),\left(\alpha_{h} \otimes h\right)\left(u_{i, j}\left(h^{-1} g^{\prime}\right)\right)\right\rangle\left(\alpha_{h} \otimes h\right)\left(u_{i, j}\left(h^{-1} g^{-1}\right)\right) \\
& =\sum_{h \in H, g^{\prime} \in \Sigma}\left\langle x\left(g^{\prime}\right), h \cdot u_{i, j}\left(h^{-1} g^{\prime}\right)\right\rangle h \cdot u_{i, j}\left(h^{-1} g\right) .
\end{aligned}
$$

Then, by the definition of $\left\{u_{i, j}\right\}_{i, j}$, we may conclude that

$$
\sum_{i=1}^{n} \sum_{j=1}^{p_{i}}{ }_{P}\left\langle x, u_{i, j}\right\rangle u_{i, j}=x .
$$

Next, we compute the left index of $\hat{V}$ :

$$
\left\langle u_{i, j}, u_{i, j}\right\rangle_{Q}(k)=\sum_{g \in \Sigma}\left\langle u_{i, j}\left(g k^{-1}\right) \cdot k, u_{i, j}(g)\right\rangle .
$$

Then if $k=e$, the right hand side is 1 , and 0 otherwise. When the support of $V$ consists only of one $H-K$ double coset, the dimensions of fibers are constant $|V|$. Then we have

$$
\text { l-Ind }[\hat{V}]=\sum_{i=1}^{n} \sum_{j=1}^{p_{i}}\left\langle u_{i, j}, u_{i, j}\right\rangle_{Q}=n|V| \cdot I .
$$

Similarly, we have $\mathrm{r}-\operatorname{Ind}[\hat{V}]=m|V| \cdot I$, and $\operatorname{Ind}[\hat{V}]=m n|V|^{2} \cdot I$. We can argue similarly about the right $B$-basis. Finally, by the computation of left index, we can show that $\hat{V}$ is left full. Similarly, $\hat{V}$ is right full.

Remark. For the computation of indices, it is not necessary to assume any outerness of the action $\alpha$ on $A$.

Let $G$ be a finite group, and assume that there exist two subgroup $H$ and $K$ of $G$ such that $G=H K$. Let $V={ }_{H} V_{K}$ be an $H$ - $K$ bundle whose fibers consists only of scalars. Let $\alpha$ be an action of $G$ on a unital $\mathrm{C}^{*}$-algebra $A$. Let $E$ and $F$ be two conditional expectations from $A \rtimes_{\alpha} G$ to $A \rtimes_{\alpha} H$ and from $A \rtimes_{\alpha} G$ to $A \rtimes_{\alpha} K$ given by the restriction. Then $\hat{V}$ is equal to a $\mathrm{C}^{*}$-algebra $A \rtimes_{\alpha} G$, and this Hilbert $\mathrm{C}^{*}$-bimodule corresponds to the example given by $E$ and $F$ before Lemma 1.11.

Proposition 1.30. Let $X={ }_{A} X_{B}$ and ${ }_{B} Y_{C}$ be Hilbert $C^{*}$-bimodules of finite type. If the centers of $A, B$ and $C$ are scalars, then

$$
\operatorname{Ind}\left[X \otimes_{B} Y\right]=\operatorname{Ind}[X] \operatorname{Ind}[Y] .
$$

Proof. As in the proof of Lemma 1.22, we have

$$
\begin{aligned}
\mathrm{r}-\operatorname{Ind}\left[X \otimes_{B} Y\right] & =\sum_{i=1}^{n} \sum_{j=1}^{m}{ }_{A}\left\langle u_{i} \otimes v_{j}, u_{i} \otimes v_{j}\right\rangle \\
& =\sum_{i=1}^{n} \sum_{j=1}^{m}{ }_{A}\left\langle u_{i B}\left\langle v_{j}, v_{j}\right\rangle, u_{i}\right\rangle=\mathrm{r}-\operatorname{Ind}[X] \mathrm{r}-\operatorname{Ind}[Y] .
\end{aligned}
$$

We have the similar formula for the left index. Therefore the index is also multiplicative. 


\section{Schur's Lemma and Frobenius Reciprocity}

Schur's Lemma and Frobenius reciprocity are the most fundamental materials in representation theory, category theory and bimodule theory. In this section, we consider them.

Lemma 2.1. Let $X={ }_{A} X$ be a Hilbert left $A$-module of finite type. Take a positive invertible operator $H$ in ${ }_{A} \mathbf{L}\left({ }_{A} X\right)$, and define a new $A$-valued inner product by

$$
{ }_{A}\langle x, y\rangle^{\sim}={ }_{A}\langle H x, y\rangle, \quad x, y \in X .
$$

We write $X^{\sim}$ as the left $A$-module with this left $A$-valued inner product. Then ${ }_{A} X^{\sim}$ is also of finite type.

Conversely, for any A-valued inner product ${ }_{A}\langle,\rangle^{\prime},{ }_{A} X^{\prime}$ with this A-valued inner product is of this form if this is of finite type.

This correspondence is one to one, and all norms given by these A-valued inner products are mutually equivalent.

Proof. We prove this proposition using the self-duality of ${ }_{A} X$. We omit the details.

Lemma 2.2. Let $X={ }_{A} X_{B}$ be a Hilbert $C^{*}$-bimodule of finite type, and let $H \in$ ${ }_{A} \operatorname{End}_{B}\left({ }_{A} X_{B}\right)$ be a positive invertible operator in ${ }_{A} \mathbf{L}\left({ }_{A} X\right)$. We introduce a left $A$ valued inner product ${ }_{A}\left\langle, \gamma^{\sim}\right.$ by ${ }_{A}\langle x, y\rangle^{\sim}={ }_{A}\langle H x, y\rangle$. We denote by ${ }_{A} X^{\sim}{ }_{B}$ the Hilbert $C^{*}$-bimodule obtained by replacing the left $A$-valued inner product by ${ }_{A}\left\langle,{ }^{-}\right.$. Then ${ }_{A} X^{\sim}{ }_{B}$ is also a Hilbert $C^{*}$-bimodule of finite type. Conversely, all ${ }_{A} X^{\sim}{ }_{B}$ 's as above are of this form, and this correspondence is one to one.

Proof. Almost all parts follow from the preceding lemma. We only need to show that $\rho^{\sim}: B \rightarrow{ }_{A} \mathbf{L}\left({ }_{A} X^{\sim}\right), \rho^{\sim}(b) x=x b$ is *-preserving with respect to ${ }_{A}\langle, \gamma$. But

$$
\begin{aligned}
{ }_{A}\left\langle x, \rho^{\sim}\left(b^{*}\right) y\right\rangle^{\sim} & ={ }_{A}\left\langle x, y b^{*} \gamma^{\sim}={ }_{A}\left\langle H x, y b^{*}\right\rangle\right. \\
& ={ }_{A}\langle(H x) b, y\rangle={ }_{A}\langle H(x b), y\rangle={ }_{A}\langle x b, y\rangle^{\sim} \\
& ={ }_{A}\left\langle\rho^{\sim}(b) x, y\right\rangle^{\sim}={ }_{A}\left\langle x, \rho^{\sim}(b)^{*} y\right\rangle^{\sim} .
\end{aligned}
$$

Proposition 2.3. Let $X={ }_{A} X_{B}$ be a Hilbert $C^{*}$-bimodule of finite type. We consider a new finite type Hilbert $A-B$ bimodule $X^{\sim}$ with the same $A-B$ actions and with two inner products ${ }_{A}\left\langle, \gamma^{\sim}\right.$ and $\langle,\rangle_{B}$. Then there exist two operators $H$ and $K$ in ${ }_{A} \operatorname{End}_{B}\left({ }_{A} X_{B}\right)$ such that ${ }_{A}\langle x, y\rangle^{\sim}={ }_{A}\langle H x, y\rangle$ and $\langle x, y\rangle_{B} \tilde{}=\langle x, K y\rangle_{B}$. In particular, if ${ }_{A} \operatorname{End}_{B}\left({ }_{A} X_{B}\right)$ is scalar, the inner products are determined up to positive constants.

Proof. This follows from Lemma 2.2.

We describe Schur's lemma.

Theorem 2.4. Let $X$ and $Y$ be a Hilbert $A-B$ bimodules of finite type. We assume that ${ }_{A} \operatorname{End}_{B}\left({ }_{A} X_{B}\right)=\mathbf{C}$ and ${ }_{A} \operatorname{End}_{B}\left({ }_{A} Y_{B}\right)=\mathbf{C}$. Then ${ }_{A} \operatorname{Hom}_{B}\left({ }_{A} X_{B},{ }_{A} Y_{B}\right)$ is of dimension one if and only if ${ }_{A} X_{B}$ is similar to ${ }_{A} Y_{B}$. Otherwise ${ }_{A} \operatorname{Hom}_{B}\left({ }_{A} X_{B},{ }_{A} Y_{B}\right)$ $=\{0\}$. Moreover, if $X$ and $Y$ are similar and both are normalized, they are unitarily equivalent. 
Proof. We assume that there exists a non-zero $T \in{ }_{A} \operatorname{Hom}_{B}\left({ }_{A} X_{B},{ }_{A} Y_{B}\right)$. Then $T^{*} T \in{ }_{A} \operatorname{End}_{B}\left({ }_{A} X_{B}\right)=\mathbf{C}$, and so $T^{*} T=\lambda I$ for some $\lambda>0$. Similarly, we have $T T^{*}=\mu I$ for some $\mu>0$. Then $T$ is invertible, i.e. $T^{-1}=\lambda^{-1} T^{*}$. Hence ${ }_{A} X_{B}$ and ${ }_{A} Y_{B}$ are similar.

Let $T$ and $S$ be elements in ${ }_{A} \operatorname{Hom}_{B}\left({ }_{A} X_{B},{ }_{A} Y_{B}\right)$ with $T \neq 0$. Then, by the assumption of irreducibility, $T S^{*}$ and $S^{*} T$ are scalar. Therefore $S$ is a scalar multiple of $T$. Let $X$ be irreducible and normalized. Then two systems of inner products on $X$ are proportional by some common positive constant $C$ :

$$
{ }_{A}\langle x, y\rangle^{\sim}=C_{A}\langle x, y\rangle, \quad\langle x, y\rangle_{B} \tilde{}=C\langle x, y\rangle_{B} .
$$

This shows the last statement.

Next, we consider the Frobenius reciprocity theorem.

Proposition 2.5. Let $X$ be a Hilbert $A-B$ bimodule of finite type, and let $Y$ be a Hilbert $C$ - $B$ bimodule of finite type. Then there exists an $A$ - $C$ bimodule isomorphism $\varphi$ from $X \otimes_{B} \bar{Y}$ to $\mathbf{L}_{B}\left({ }_{C} Y_{B},{ }_{A} X_{B}\right)$ such that

$$
\varphi(p \otimes \bar{q})(y)=p\langle q, y\rangle_{B},
$$

where $\mathbf{L}_{B}\left({ }_{C} Y_{B},{ }_{A} X_{B}\right)$ is viewed as an $A-C$ bimodule as follows:

$$
(a \cdot f \cdot c)(y)=a f(c y) .
$$

Let $X$ be a Hilbert $B$ - $A$ bimodule of finite type, and let $Y$ be a Hilbert $B-C$ bimodule of finite type. Then there exists an $A-C$ bimodule isomorphism $\varphi^{\prime}$ from $\bar{X} \otimes_{B} Y$ to ${ }_{B} \mathbf{L}\left({ }_{B} X_{A},{ }_{B} Y_{C}\right)$ such that

$$
\varphi^{\prime}(\bar{p} \otimes q)(x)={ }_{B}\langle x, p\rangle q,
$$

where ${ }_{B} \mathbf{L}\left({ }_{B} X_{A},{ }_{B} Y_{C}\right)$ is viewed as an $A-C$ bimodule as follows:

$$
(a \cdot g \cdot c)(x)=g(x a) c .
$$

Proof. We prove only the first statement. Let $\left\{v_{i}\right\}_{i}$ be a right $B$-basis in ${ }_{C} Y_{B}$. For $f \in \mathbf{L}_{B}\left({ }_{C} Y_{B},{ }_{A} X_{B}\right)$ we define $\psi(f)$ as follows:

$$
\psi(f)=\sum_{i} f\left(v_{i}\right) \otimes \bar{v}_{i} \in X \otimes_{B} \bar{Y} .
$$

Then, for all $z \in{ }_{C} Y_{B}$,

$$
\begin{aligned}
(\varphi \circ \psi)(f)(z) & =\varphi\left(\sum_{i=1}^{n} f\left(v_{i}\right) \otimes \bar{v}_{i}\right)(z)=\sum_{i=1}^{n} f\left(v_{i}\right)\left\langle v_{i}, z\right\rangle_{B} \\
& =f\left(\sum_{i=1}^{n} v_{i}\left\langle v_{i}, z\right\rangle_{B}\right)=f(z) .
\end{aligned}
$$

Conversely,

$$
\begin{aligned}
& (\psi \circ \varphi)(x \otimes \bar{y})=\psi\left(z \mapsto x\langle y, z\rangle_{B}\right)=\sum_{i=1}^{n} x\left\langle y, v_{i}\right\rangle_{B} \otimes \bar{v}_{i} \\
& =x \otimes\left(\sum_{i=1}^{n}\left\langle y, v_{i}\right\rangle_{B} \bar{v}_{i}\right)=x \otimes\left(\sum_{i=1}^{n} \overline{v_{i}\left\langle v_{i}, y\right\rangle_{B}}\right)=x \otimes \bar{y} .
\end{aligned}
$$

Let $A$ be a unital $\mathrm{C}^{*}$-algebra and $X$ a Hilbert $A$ - $A$ bimodule. 
Definition 2.6. $x \in X$ is called an A-central element if $a x=x a$ for all $a \in A$. We denote by $X^{A}$ the set of all $A$-central elements in $X$.

The following two theorems are called Frobenius reciprocity theorems.

Theorem 2.7. Let $A, B$ be unital $C^{*}$-algebras, and let ${ }_{A} X_{B},{ }_{A} Y_{B}$ be Hilbert $C^{*}$ bimodules of finite type. Then there exists a $\mathbf{C}$-linear isomorphism

$$
\tilde{\varphi}:\left(X \otimes_{B} \bar{Y}\right)^{A} \rightarrow{ }_{A} \operatorname{Hom}_{B}\left({ }_{A} Y_{B},{ }_{A} X_{B}\right) .
$$

In particular, $\tilde{\varphi}\left(\sum_{i} x_{i} \otimes \overline{y_{i}}\right)(u)=\sum_{i} x_{i}\left\langle y_{i}, u\right\rangle_{B}$. There also exists a $\mathbf{C}$-linear isomorphism

$$
\phi^{\prime}:\left(\bar{Y} \otimes_{A} X\right)^{B} \rightarrow{ }_{A} \operatorname{Hom}_{B}\left({ }_{A} Y_{B},{ }_{A} X_{B}\right)
$$

such that $\phi^{\prime}\left(\sum_{i} \overline{y_{i}} \otimes x_{i}\right)(u)=\sum_{i} A\left\langle u, y_{i}\right\rangle x_{i}$.

Proof. Theorem 2.7 follows from the previous Proposition 2.5.

Theorem 2.8. Let $A, B$ and $C$ be unital $C^{*}$-algebras, and let ${ }_{A} X_{B},{ }_{B} Y_{C}$ and ${ }_{A} Z_{C}$ be Hilbert $C^{*}$-bimodules of finite type. Then the followings are isomorphisms of C-vector spaces:

(1) ${ }_{A} \operatorname{Hom}_{C}\left({ }_{A} Z_{C},{ }_{A}\left(X \otimes_{B} Y\right)_{C}\right) \simeq{ }_{A} \operatorname{Hom}_{B}\left({ }_{A}\left(Z \otimes_{C} \bar{Y}\right)_{B},{ }_{A} X_{B}\right)$,

(2) ${ }_{A} \operatorname{Hom}_{C}\left({ }_{A} Z_{C},{ }_{A}\left(X \otimes_{B} Y\right)_{C}\right) \simeq{ }_{B} \operatorname{Hom}_{C}\left({ }_{B}\left(\bar{X} \otimes_{A} Z\right)_{C},{ }_{B} Y_{C}\right)$.

Proof. We only prove (1):

$$
\begin{aligned}
{ }_{A} \operatorname{Hom}_{C}\left({ }_{A} Z_{C},{ }_{A}\left(X \otimes_{B} Y\right)_{C}\right) & \simeq\left(\left({ }_{A}\left(X \otimes_{B} Y\right)_{C}\right) \otimes_{C} \bar{Z}_{A}\right)^{A} \\
& \simeq\left({ }_{A} X_{B} \otimes\left({ }_{B}\left(Y \otimes_{C} \bar{Z}\right){ }_{A}\right)\right)^{A} \\
& \simeq{ }_{A} \operatorname{Hom}_{B}\left({ }_{A}\left(Z \otimes_{C} \bar{Y}\right)_{B},{ }_{A} X_{B}\right) .
\end{aligned}
$$

We use $\overline{(X \otimes Y)}=\bar{Y} \otimes \bar{X}$.

We have already defined the left and right adjoints of $T \in{ }_{A} \operatorname{Hom}_{B}\left({ }_{A} X_{B}\right)$ by inner products. We can also define ${ }^{*} T$ and $T^{*}$ by the isomorphisms $\tilde{\varphi}$ and $\varphi^{\prime}$ in the Frobenius Reciprocity Theorem 2.7 as follows. Suppose that $T=\Theta_{x, y}$. Then $T^{*}=\Theta_{y, x}$ can be also obtained by

$$
\begin{gathered}
{ }_{A} \operatorname{Hom}_{B}\left({ }_{A} X_{B},{ }_{A} Y_{B}\right) \simeq\left({ }_{A}\left(Y \otimes{ }_{B} \bar{X}\right){ }_{A}\right)^{A} \rightarrow\left({ }_{A}\left(X \otimes \otimes_{B} \bar{Y}\right){ }_{A}\right)^{A} \simeq{ }_{A} \operatorname{Hom}_{B}\left({ }_{A} Y_{B},{ }_{A} X_{B}\right), \\
T \simeq y \otimes \bar{x} \rightarrow x \otimes \bar{y} \simeq T^{*} .
\end{gathered}
$$

Similarly, suppose that $S=\tilde{\Theta}_{x, y}$. Then ${ }^{*} S=\tilde{\Theta}_{y, x}$ can be obtained by

$$
\begin{gathered}
{ }_{A} \operatorname{Hom}_{B}\left({ }_{A} X_{B},{ }_{A} Y_{B}\right) \simeq\left({ }_{B} \bar{X}_{A} \otimes{ }_{A} Y_{B}\right)^{B} \rightarrow\left({ }_{B} \bar{Y}_{A} \otimes{ }_{A} X_{B}\right)^{B} \simeq{ }_{A} \operatorname{Hom}_{B}\left({ }_{A} Y_{B},{ }_{A} X_{B}\right), \\
S \simeq \bar{x} \otimes y \rightarrow \bar{y} \otimes x \simeq * S .
\end{gathered}
$$

In general the right adjoint $T^{*}$ and the left adjoint ${ }^{*} T$ need not coincide.

Definition 2.9. Let $A$ and $B$ be unital $\mathrm{C}^{*}$-algebras, and let $X$ be a Hilbert $A$-B bimodule of finite type. We call $X$ semisimple if for $T \in{ }_{A} \operatorname{End}_{B}\left({ }_{A} X_{B}\right), T^{*}$ and ${ }^{*} T$ coincide.

Theorem 2.10. Let ${ }_{A} X_{B}$ be a semisimple Hilbert $C^{*}$-bimodule of finite type. Then ${ }_{A} X_{B}$ is decomposed into a direct sum of finitely many irreducible bimodules, and this decomposition is unique up to unitary equivalence. 
Proof. There exists a one-to-one correspondence between direct summands of ${ }_{A} X_{B}$ and selfadjoint projections in ${ }_{A} \operatorname{End}_{B}\left({ }_{A} X_{B}\right)$. Since ${ }_{A} X_{B}$ is assumed to be of finite type, ${ }_{A} \operatorname{End}_{B}\left({ }_{A} X_{B}\right)$ is finite dimensional. A direct summand $p\left({ }_{A} X_{B}\right)$ is irreducible if and only if $p$ is a minimal projection. Let $p, q \in{ }_{A} \operatorname{End}_{B}\left({ }_{A} X_{B}\right)$ be selfadjoint projections. Then $p$ and $p$ are unitarily equivalent in ${ }_{A} \operatorname{End}_{B}\left({ }_{A} X_{B}\right)$ if and only if $p\left({ }_{A} X_{B}\right)$ and $q\left({ }_{A} X_{B}\right)$ are unitarily equivalent as Hilbert $\mathrm{C}^{*}$-bimodules.

Remark. The semisimplicity is not necessary for Frobenius reciprocity.

If $X$ is irreducible, $X$ is clearly semisimple, and if $X$ and $Y$ are semisimple, $X \oplus Y$ is also semisimple.

Example. Let $X=\mathbf{C}^{n}, A=\mathbf{C}, B=\mathbf{C}$. Let the $A$-inner product be the ordinary inner product, and let the $B$-inner product be given by some positive invertible matrix $h$ which is not a scalar operator. Then their two adjoints do not coincide.

\section{Minimality}

For an inclusion of factors $N \subset M$, as in Havet (18]), Hiai ([19]) and Longo ([34]), there exists a conditional expectation $E_{0}$ from $M$ to $N$ such that Index $\left[E_{0}\right] \leq$ Index $[E]$ for every conditional expectation $E$ from $M$ to $N$. This expectation is called the minimal (or minimum) conditional expectation. The second named author (47]) considered minimal the conditional expectation for $\mathrm{C}^{*}$-algebras, modifying the argument of Hiai ([19]). Kosaki and Longo showed the multiplicativity of the minimal index for subfactors first in 32, 36. Kawakami and Watatani ([28]) obtained the same multiplicativity for simple $\mathrm{C}^{*}$-algebras. In this chapter, we treat the minimality of Hilbert $\mathrm{C}^{*}$-bimodules corresponding to those in inclusion relations.

We start by giving the known characterization of minimal index for $\mathrm{C}^{*}$-inclusions.

Proposition 3.1 ([28, Proposition 2]). Let $A \subset B$ be a unital inclusion of unital $C^{*}$-algebras with $Z(A)=\mathbf{C} \cdot I, Z(B)=\mathbf{C} \cdot I$. Assume that there exists conditional expectations $F: B \rightarrow A$ of index-finite type. Then, there exists a unique minimal conditional expectation $E_{0}: B \rightarrow A$, i.e. Index $E_{0} \leq$ Index $E$ for any conditional expectation $E: B \rightarrow A$. Moreover, $E=E_{0}$ if and only if

$$
\sum_{i=1}^{n} u_{i} x u_{i}^{*}=C E(x) \quad\left(x \in A^{\prime} \cap B\right)
$$

for some constant $C>0$, where $\left\{u_{1}, u_{2}, \ldots, u_{n}\right\}$ is a (quasi) basis for $E$.

Following this formulation, we define the minimality of Hilbert $\mathrm{C}^{*}$-bimodules. The following characterization in the case of von Neumann algebra is given in [9].

Proposition 3.2. Let ${ }_{A} X_{B}$ be a Hilbert $C^{*}$-bimodule of finite type. We assume $Z(A)=\mathbf{C} \cdot I$ and $Z(B)=\mathbf{C} \cdot I$. Let $\left\{u_{i}\right\}_{i}$ be a right B-basis in $X$ and $\left\{v_{j}\right\}_{j}$ be a left $A$-basis in $X$. We define conditional expectations $F$ from $\mathbf{L}_{B}\left(X_{B}\right)$ to $A$ and $G$ from ${ }_{A} \mathbf{L}\left({ }_{A} X\right)$ to $B$ as follows:

$$
\begin{aligned}
& F(T)=(\mathrm{r}-\operatorname{Ind}[X])^{-1} \sum_{i}\left\langle T u_{i}, u_{i}\right\rangle, \\
& G(S)=(\operatorname{l-Ind}[X])^{-1} \sum_{j}\left\langle v_{j}, S v_{j}\right\rangle_{B} .
\end{aligned}
$$

Then the following are equivalent. 
(1) $F$ is a minimal expectation.

(2) $G$ is a minimal expectation.

(3) There exists a positive constant $C$ such that, for all $T \in{ }_{A} \operatorname{End}_{B}\left({ }_{A} X_{B}\right)$,

$$
\sum_{i} A\left\langle T u_{i}, u_{i}\right\rangle=C \sum_{j}\left\langle v_{j}, T v_{j}\right\rangle_{B}
$$

as an equality of scalars.

Moreover, if (3) holds, $C$ is equal to $\mathrm{r}-\operatorname{Ind}[X](1-\operatorname{Ind}[X])^{-1}$.

Proof. We show the equivalence between (1) and (3). We remark that by Lemma 1.26, $\left\{\sqrt{\mathrm{r}-\operatorname{Ind}[X]} \Theta_{u_{i}, v_{j}}\right\}_{i, j}$ is a quasi basis for $F$. By Proposition 3.1, $F$ is minimal if and only if there exists a positive constant $K$ such that, for all $T \in A^{\prime} \cap \mathbf{L}_{B}\left(X_{B}\right)=$ ${ }_{A} \operatorname{End}_{B}\left({ }_{A} X_{B}\right)$,

$$
\sum_{i} \sum_{j}(\mathrm{r}-\operatorname{Ind}[X]) \Theta_{u_{i}, v_{j}} T \Theta_{u_{i}, v_{j}}^{*}=K F(T) .
$$

On the other hand, for all $z \in X$,

$$
\begin{aligned}
\sum_{i} \sum_{j} \Theta_{u_{i}, v_{j}} T \Theta_{u_{i}, v_{j}}^{*}(z) & =\sum_{i} \sum_{j} \Theta_{u_{i}, v_{j}} T v_{j}\left\langle u_{i}, z\right\rangle_{B} \\
& =\sum_{i} \sum_{j} u_{i}\left\langle v_{j}, T v_{j}\right\rangle_{B}\left\langle u_{i}, z\right\rangle_{B} \\
& =\sum_{i} u_{i}\left(\sum_{j}\left\langle v_{j}, T v_{j}\right\rangle_{B}\right)\left\langle u_{i}, z\right\rangle_{B} \\
& =\left(\sum_{i} u_{i}\left\langle u_{i}, z\right\rangle_{B}\right) \sum_{j}\left\langle v_{j}, T v_{j}\right\rangle_{B} \\
& =\sum_{j} z\left\langle v_{j}, T v_{j}\right\rangle_{B} .
\end{aligned}
$$

The equivalence between (2) and (3) is similar.

If we put $T=I$, we can get the value of the constant $C$.

Corollary 3.3. Let $A$ and $B$ be unital $C^{*}$-algebras, and assume that $Z(A)=\mathbf{C} \cdot I$ and $Z(B)=\mathbf{C} \cdot I$. If $X$ is irreducible, then $X$ is minimal.

Corollary 3.4. Let $A$ and $B$ be unital $C^{*}$-algebras, and $A \subset B$ a unital inclusion. Let $E$ be a minimal expectation from $B$ to $A$. Then the dual expectation $\tilde{E}$ from the basic $C^{*}$-extension to $B$ is also minimal. Moreover, let $B_{i}$ be the ith $C^{*}$-basic extension, and $E_{i}$ the expectation from $B_{i}$ to $B_{i-1}$ canonically defined. Then all $E_{i}$ 's are also minimal.

Proof. Let $X={ }_{A} B_{B}$ be as in the example after Definition 6 . Then $\mathbf{L}_{B}\left(X_{B}\right)$ is equal to $B$ and ${ }_{A} \mathbf{L}\left({ }_{A} X\right)$ is equal to the $\mathrm{C}^{*}$-basic extension $B_{1}$. $E$ is an expectation from $\mathbf{L}_{B}\left(X_{B}\right)$ to $A$, and $F$ is an expectation from ${ }_{A} \mathbf{L}\left({ }_{A} X\right)$ to $B$. Then by the previous proposition, $E$ is minimal if and only if $F$ is minimal. The last statement is clear.

Definition 3.5. If ${ }_{A} X_{B}$ satisfies (1)-(3) of the previous proposition, we call ${ }_{A} X_{B}$ a minimal Hilbert $C^{*}$-bimodule. 
When we consider the minimality of a Hilbert $\mathrm{C}^{*}$-bimodule, we assume that the centers of all $\mathrm{C}^{*}$-algebras consist only of scalars.

When ${ }_{A} X_{B}$ is not minimal, we can 'remetrize' $X$ and make it into a minimal bimodule.

Lemma 3.6. Let $A$ and $B$ be unital $C^{*}$-algebras, $Z(A)=\mathbf{C} \cdot I, Z(B)=\mathbf{C} \cdot I$, and let ${ }_{A} X_{B}$ be a Hilbert $C^{*}$-bimodule of finite type. Then there exists a left $A$-valued inner product ${ }_{A}\langle,\rangle^{0}$ such that a bimodule ${ }_{A} X_{B}^{0}$ obtained by replacing the original left A-inner product by ${ }_{A}\langle,\rangle^{0}$ is minimal. Moreover, such a left inner product is unique up to positive constants. In particular, we may take this inner product such that $\mathrm{r}-\operatorname{Ind}\left[X^{0}\right]=\mathrm{r}-\operatorname{Ind}[X]$.

Proof. Let $\left\{u_{i}\right\}_{i}$ be a right $B$-basis in $X$ and $\left\{v_{j}\right\}_{j}$ a left $A$-basis in $X$. Let $E$ be the conditional expectation from $\mathbf{L}_{B}\left(X_{B}\right)$ to $A$ given by $\left\{u_{i}\right\}_{i}$. Then by Lemma $1.26 E$ is of index finite type. By Theorem 2.12.3 in [47, there exists a minimal expectation $E_{0}$ from $\mathbf{L}_{B}\left(X_{B}\right)$ to $A$. Moreover, by Proposition 1.4.1 in [47], there exists an $H \in A^{\prime} \cap \mathbf{L}_{B}\left(X_{B}\right)={ }_{A} \operatorname{End}_{B}\left({ }_{A} X_{B}\right)$ such that $E_{0}(T)=E(H T)$ for $T \in \mathbf{L}_{B}\left(X_{B}\right)$. We remark that

$$
E_{0}\left(\Theta_{x, y}\right)=E\left(H \Theta_{x, y}\right)=E\left(\Theta_{H x, y}\right)={ }_{A}\langle H x, y\rangle .
$$

Since ${ }_{A}\langle H x, x\rangle=E_{0}\left(\Theta_{x, x}\right) \geq 0, H$ is positive. By Proposition 1.4.1(2) in [47], $H$ is invertible and contained in $A^{\prime} \cap \mathbf{L}_{B}\left(X_{B}\right)={ }_{A} \operatorname{End}_{B}\left({ }_{A} X_{B}\right)$. Then $H$ is invertible in the $\mathrm{C}^{*}$-algebra ${ }_{A} \mathbf{L}\left({ }_{A} X\right)$.

We define a new left $A$-valued inner product ${ }_{A}\langle$,$\rangle as follows:$

$$
{ }_{A}\langle x, y\rangle^{0}={ }_{A}\langle H x, y\rangle \text {. }
$$

We denote by ${ }_{A} X_{B}^{0}$ the Hilbert $\mathrm{C}^{*}$-bimodule of finite type determined by this new left inner product. We define a conditional expectation ${ }^{0} E: \mathbf{L}_{B}\left(X_{B}^{0}\right) \rightarrow A$ as follows:

$$
{ }^{0} E(T)=\left(\mathrm{r}-\operatorname{Ind}\left[X^{0}\right]^{-1} \sum_{i}{ }_{A}\left\langle T u_{i}, u_{i}\right\rangle^{0}\right.
$$

Then

$$
\begin{aligned}
\mathrm{r}-\operatorname{Ind}\left[X^{0}\right] & =\sum_{i}{ }_{A}\left\langle u_{i}, u_{i}\right\rangle^{0}=\sum_{i}{ }_{A}\left\langle H u_{i}, u_{i}\right\rangle \\
& =(\mathrm{r}-\operatorname{Ind}[X]) E(H)=(\mathrm{r}-\operatorname{Ind}[X]) E_{0}(I)=\mathrm{r}-\operatorname{Ind}[X] .
\end{aligned}
$$

Moreover, for $T \in \mathbf{L}_{B}\left(X_{B}\right)=\mathbf{L}_{B}\left(X_{B}^{0}\right)$,

$$
\begin{aligned}
E_{0}(T) & =E(H T)=(\mathrm{r}-\operatorname{Ind}[X])^{-1} \sum_{i}{ }_{A}\left\langle H T u_{i}, u_{i}\right\rangle \\
& =\left(\mathrm{r}-\operatorname{Ind}\left[X^{0}\right]\right)^{-1} \sum_{i}{ }_{A}\left\langle T u_{i}, u_{i}\right\rangle^{0}={ }^{0} E(T) .
\end{aligned}
$$

Since ${ }^{0} E=E_{0}$ is minimal, ${ }_{A} X_{B}^{0}$ is a minimal bimodule.

Suppose the other Hilbert $\mathrm{C}^{*}$-bimodule ${ }_{A} X^{\sim}{ }_{B}$ defined by replacing only the left $A$-inner product of ${ }_{A} X_{B}$ by ${ }_{A}\left\langle, \gamma^{\sim}\right.$ is also minimal. Then by Proposition 2.3 , there exists a positive invertible element $K \in{ }_{A} \operatorname{End}_{B}\left({ }_{A} X_{B}\right)$ such that

$$
{ }_{A}\langle x, y\rangle^{\sim}={ }_{A}\langle K x, y\rangle \quad(x, y \in X) .
$$


Then we may define a conditional expectation $\tilde{E}: \mathbf{L}_{B}\left(X_{B}^{\sim}\right) \rightarrow A$ by

$$
\tilde{E}(T)=(\mathrm{r}-\operatorname{Ind}[\tilde{X}])^{-1} \sum_{i}{ }_{A}\left\langle T u_{i}, u_{i}\right\rangle
$$

Since $\tilde{X}$ is minimal, $\tilde{E}$ is also minimal. By Theorem 2.12.3 in [47], minimal conditional expectations are unique. Identifying $\mathbf{L}_{B}\left(X_{B}\right)=\mathbf{L}_{B}\left(\left(X^{\sim}\right)_{B}\right)$, we have $E^{0}=E$. By this

$$
\left(\mathrm{r}-\operatorname{Ind}\left[X^{0}\right]\right)_{A}^{-1}\langle x, y\rangle^{0}=\left(\mathrm{r}-\operatorname{Ind}\left[X^{\sim}\right]\right)^{-1}\langle x, y\rangle .
$$

Then, for some positive constant $C$,

$$
{ }_{A}\langle x, y\rangle^{0}=C_{A}\langle x, y\rangle .
$$

Proposition 3.7. Let $X={ }_{A} X_{B}$ be a Hilbert $C^{*}$-bimodule of finite type. We assume that we have another left $A$-valued inner product ${ }_{A}\left\langle, \gamma^{\sim}\right.$ on $X$. We denote by ${ }_{A} X_{B}^{\sim}$ the Hilbert $A-B$ bimodule obtained by replacing the left $A$-bimodule by ${ }_{A}\langle$,$\rangle .$ We assume that ${ }_{A} X^{\sim}{ }_{B}$ is also of finite type. Then the inclusions $A \subset \mathbf{L}_{B}\left(X_{B}\right)$ and $A \subset \mathbf{L}_{B}\left(X_{B}^{\sim}\right)$ coincide. Moreover, the inclusions $B \subset{ }_{A} \mathbf{L}\left({ }_{A} X\right)$ and $B \subset{ }_{A} \mathbf{L}\left({ }_{A} X^{\sim}\right)$ are conjugate.

Proof. Since the actions and the right $B$-inner products are the same, it is clear that the inclusions $A \subset \mathbf{L}_{B}\left(X_{B}\right)$ and $A \subset \mathbf{L}_{B}\left(X_{B}^{\sim}\right)$ are the same.

By Proposition 2.3, there exists a positive invertible operator $H \in{ }_{A} \operatorname{End}_{B}\left({ }_{A} X_{B}\right)$ such that

$$
{ }_{A}\langle x, y\rangle^{\sim}={ }_{A}\langle H x, y\rangle, \quad x, y \in X .
$$

We define a linear map $U: X^{\sim} \rightarrow X$ by $U x=H^{1 / 2} x$ for $x \in X$. Then $U \in$ ${ }_{A} \operatorname{Hom}_{B}\left({ }_{A} X^{\sim}{ }_{B},{ }_{A} X_{B}\right)$. Since $H^{1 / 2}$ is invertible, $U$ is a bijection. Moreover, $U$ conserves left $A$-inner products, i.e., ${ }_{A}\langle U x, U y\rangle={ }_{A}\langle x, y\rangle$. Finally, we define $\varphi$ : ${ }_{A} \mathbf{L}\left({ }_{A} X\right) \rightarrow{ }_{A} \mathbf{L}\left({ }_{A} X\right)$ by

$$
\varphi(T)=U T U^{-1}, \quad T \in{ }_{A} \mathbf{L}\left({ }_{A} X\right) .
$$

This $\varphi$ gives the desired conjugation.

Lemma 3.8. Let ${ }_{A} X_{B}$ be a minimal Hilbert $C^{*}$-bimodule of finite type. If $p$ is an element in ${ }_{A} \operatorname{End}_{B}\left({ }_{A} X_{B}\right)$ which is self adjoint with respect to two inner products, then $p X$ is also minimal.

Proof. This is clear from Proposition 3.2.

We assume that $Z(A)$ and $Z(B)$ consist of scalars. We take $d(X)$ to be a positive scalar such that $\operatorname{Ind}[X]=d(X)^{2} \cdot I$ and call this the dimension of $X$. It is clear that $d(X \otimes Y)=d(X) d(Y)$ by Proposition 1.30, and $d(\bar{X})=d(X)$. But the additivity of the dimension is related to the minimality.

Lemma 3.9. Let $A$ and $B$ be unital $C^{*}$-algebras, and let ${ }_{A} X_{B}$ and ${ }_{A} Y_{B}$ be Hilbert $A-B$ bimodules of finite type. We assume that $Z(A)=\mathbf{C} \cdot I, Z(B)=\mathbf{C} \cdot I$. Then

$$
d(X \oplus Y) \geq d(X)+d(Y) .
$$

The following are equivalent.

(1) $d(X \oplus Y)=d(X)+d(Y)$. 
(2) $\frac{\mathrm{r}-\operatorname{Ind}[X]}{\mathrm{l}-\operatorname{Ind}[X]}=\frac{\mathrm{r}-\operatorname{Ind}[Y]}{\mathrm{l}-\operatorname{Ind}[Y]}$.

Proof. Let $x_{1}=\mathrm{r}-\operatorname{Ind}[X], x_{2}=\mathrm{l}-\operatorname{Ind}[X], y_{1}=\mathrm{r}-\operatorname{Ind}[Y]$ and $y_{2}=\mathrm{l}-\operatorname{Ind}[Y]$. Then

$$
\begin{aligned}
& (d(X \oplus Y))^{2}-((d(X)+d(Y)))^{2}=\left(x_{1}+x_{2}\right)\left(y_{1}+y_{2}\right)-\left(\sqrt{x_{1} x_{2}}+\sqrt{y_{1} y_{2}}\right)^{2} \\
& \quad=x_{1} y_{2}+y_{1} x_{2}-2 \sqrt{\left(x_{1} y_{2}\right)\left(y_{1} x_{2}\right)} \geq 0 .
\end{aligned}
$$

The equality holds if and only if $x_{1} y_{2}=y_{1} x_{2}$.

Lemma 3.10. We assume that $Z(A)=\mathbf{C} \cdot I$ and $Z(B)=\mathbf{C} \cdot I$. Let $\left\{X_{i}\right\}_{i}=$ $\left\{{ }_{A}\left(X_{i}\right)_{B}\right\}_{i}$ be a finite family of minimal Hilbert $C^{*}$-bimodules of finite type. Then the direct sum $X=\bigoplus_{i} X_{i}$ is minimal if and only if

$$
d(X)=\sum_{i} d\left(X_{i}\right)
$$

Conversely, let ${ }_{A} X_{B}$ be a semisimple Hilbert $C^{*}$-bimodule. Then $X$ is minimal if and only if

$$
d(X)=\sum_{i} d\left(X_{i}\right)
$$

where $X=\bigoplus_{i} X_{i}$ is the irreducible decomposition of $X$.

Proof. We take a right $B$-basis $\left\{u_{k}^{i}\right\}_{k}$ in $X_{i}$ for each $i$. Then the union of these sets forms a right $B$-basis in $X$. We let $p_{i}$ be a unit on ${ }_{A} \operatorname{End}_{B}\left({ }_{A}\left(X_{i}\right)_{B}\right)$. Then

$$
\begin{aligned}
\sum_{i} \sum_{k}{ }_{A}\left\langle T u_{k}^{i}, u_{k}^{i}\right\rangle & =\sum_{i} \sum_{k}{ }_{A}\left\langle T p_{i} u_{k}^{i}, p_{i} u_{k}^{i}\right\rangle \\
& =\sum_{i} \sum_{k}{ }_{A}\left\langle p_{i} T p_{i} u_{k}^{i}, u_{k}^{i}\right\rangle .
\end{aligned}
$$

Similarly, we take a left $A$-basis $\left\{v_{j}^{i}\right\}_{j}$ in $X_{i}$ for each $i$. Then

$$
\sum_{i} \sum_{j}\left\langle T v_{j}^{i}, v_{j}^{i}\right\rangle_{B}=\sum_{i} \sum_{j}\left\langle p_{i} T p_{i} v_{j}^{i}, v_{j}^{i}\right\rangle_{B} .
$$

We may assume that $T \in{ }_{A} \operatorname{End}_{B}\left({ }_{A}\left(X_{i}\right)_{B}\right)$ for some $i$. Then

$$
\sum_{i} \sum_{k}{ }_{A}\left\langle T u_{k}^{i}, u_{k}^{i}\right\rangle=C_{i} \sum_{i} \sum_{k}\left\langle T v_{j}^{i}, v_{j}^{i}\right\rangle_{B}
$$

for some positive constant $C_{i}$. For the minimality of $X$, all $C_{i}$ 's must be equal. If we take $p_{i}$ for $T$, we have

$$
C_{i}=\left(\mathrm{r}-\operatorname{Ind}\left[X_{i}\right]\right)\left(\mathrm{l}-\operatorname{Ind}\left[X_{i}\right]\right)^{-1} .
$$

The converse statement is proved in a similar way.

If ${ }_{A} X_{B}$ and ${ }_{A} Y_{B}$ are minimal and normalized, so is ${ }_{A} X_{B} \oplus_{A} Y_{B}$. For an arbitrary bimodule, we can take a minimal and normalized bimodule which is similar to the original bimodule. We only consider minimal and normalized bimodules when necessary.

We can consider principal graphs for inclusions of $\mathrm{C}^{*}$-algebras (or more generally for Hilbert $\mathrm{C}^{*}$-bimodules) and their Perron-Frobenius eigenvalues, as well as the subfactor case.

Let $A$ and $B$ be unital $\mathrm{C}^{*}$-algebras, $X={ }_{A} X_{B}$ a minimal Hilbert $A$ - $B$ bimodule of finite type. Let $\mathcal{G}_{A, A}$ be the equivalence classes of irreducible $A$ - $A$ bimodules 
appearing in the decomposition of ${ }_{A}(X \otimes \bar{X} \otimes \cdots \otimes \bar{X})_{A}$ 's, and let $\mathcal{G}_{A, B}$ be the equivalence classes of irreducible $A-B$ bimodules appearing in the decomposition of ${ }_{A}(X \otimes \bar{X} \otimes \cdots \otimes X)_{B}$ 's.

Let $\mathcal{G}$ be a bipartite graph having even vertices $\mathcal{G}_{A, A}$ and odd vertices $\mathcal{G}_{A, B}$ with $n$ edges between $E \in \mathcal{G}_{A, A}$ and $O \in \mathcal{G}_{A, B}$ if $E \otimes X$ contains $n$-times $O$. This $\mathcal{G}$ is called the right graph of $X$. We define the left graph of $X$ similarly.

If the object of $\mathcal{G}$ is finite, set $\mathcal{G}=\left\{V_{1}, \ldots, V_{n}\right\}$. Then we define a matrix $A \in M_{n}(\mathbf{Z})$ as follows. If we tensor $X$ or $\bar{X}$ to $V_{i}$, then $V_{j}$ is contained $p$ times, and we put $A(i, j)=p$. We remark that multiple tensor products of irreducible bimodules are minimal. Then, by the additivity and multiplicativity of $d$, the column vector ${ }^{t}\left(d\left(V_{1}\right), \ldots, d\left(V_{n}\right)\right)$ is an eigenvector of $A$ and the eigenvalue is $d(X)$. This $d(X)$ is the Perron-Frobenius eigenvalue of the irreducible positive matrix $A$. We can calculate these for simple examples in the $\mathrm{C}^{*}$-situation.

Next, we consider adjoints in $\operatorname{End}(X)$ for a minimal bimodule $X$.

Lemma 3.11. Let $A \subset B$ be a unital $C^{*}$-inclusion. We assume that $Z(A)=\mathbf{C} \cdot I$, $Z(B)=\mathbf{C} \cdot I$. Let $E$ be a conditional expectation of index finite type from $B$ to $A$. If $E$ is a minimal expectation, then $E(c b)=E(b c)$ for all $c \in A^{\prime} \cap B$ and all $b \in B$.

Proof. We take a $c \in A^{\prime} \cap B$ and fix it. We put $F(b)=E(b c)$ for $b \in B$. Then $F \in \operatorname{Hom}_{A}\left(B_{A}, A_{A}\right)$. Since $B_{A}$ is self-dual, there exists an $h \in B$ such that

$$
F(b)=\left\langle h^{*}, b\right\rangle_{A}=E(h b) .
$$

This shows that $E(h b)=E(b c)$. For all $a \in A$,

$$
E(h a b)=E(a b c)=a E(b c)=a E(h b)=E(a h b) .
$$

This shows that $E((h a-a h) b)=0$ for all $b \in B$, and so $h a=a h$. Then $h \in A^{\prime} \cap B$.

Since $E$ is minimal, the restriction of $E$ to $A^{\prime} \cap B$ is an ordinary trace. For $x \in A^{\prime} \cap B$ we have $E(h x)=E(x c)=E(c x)$. This shows that $h=c$, and so $E(c b)=E(b c)$.

Lemma 3.12. Let $A \subset B$ be a unital inclusion of $C^{*}$-algebras. Moreover, we assume that $Z(A)=\mathbf{C} \cdot I, Z(B)=\mathbf{C} \cdot I$, and $E(b c)=E(c b)$ for all $b \in B$ and all $c \in A^{\prime} \cap B$. Let $X={ }_{A} X_{B}$ be as in the example after Definition 1.6. Then $X$ is semisimple.

Proof. In this case, ${ }_{A} \operatorname{End}_{B}\left({ }_{A} X_{B}\right)=\pi\left(A^{\prime} \cap B\right)$. For $T \in{ }_{A} \operatorname{End}_{B}\left({ }_{A} X_{B}\right)$ there exists a $c \in A^{\prime} \cap B$ such that $T x=\pi(c) x=c x$. Let $x, y \in B$. Then

$$
\langle T x, y\rangle_{B}=\langle c x, y\rangle_{B}=x^{*} c^{*} y=\left\langle x, c^{*} y\right\rangle_{B} .
$$

On the other hand,

$$
{ }_{A}\langle T x, y\rangle={ }_{A}\langle c x, y\rangle=E\left(c x y^{*}\right)=E\left(x y^{*} c\right)={ }_{A}\left\langle x, c^{*} y\right\rangle .
$$

This shows that $T^{*}={ }^{*} T$.

Remark. By Lemma 3.11, if $E$ is a minimal expectation, the conclusion of Lemma 3.12 holds.

Lemma 3.13. Let ${ }_{A} X_{B}$ be a Hilbert $C^{*}$-bimodule of finite type. We assume that ${ }_{A} X_{B}$ is semisimple. We take a self adjoint projection $p$ in ${ }_{A} \operatorname{End}_{B}\left({ }_{A} X_{B}\right)$. Then $p\left({ }_{A} X_{B}\right)$ is a Hilbert $A-B$ bimodule of finite type, and $p\left({ }_{A} X_{B}\right)$ is semisimple. 
Proof. By Lemma 1.8, $p X$ is of finite type. We take $T \in{ }_{A} \operatorname{End}_{B}\left(p\left({ }_{A} X_{B}\right)\right)$. Since $X=p X+(I-p) X$ as Hilbert $A-B$ bimodule, there exists an $S \in{ }_{A} \operatorname{End}_{B}\left({ }_{A} X_{B}\right)$ such that $T=p S p$. Then $T^{*}=(p S p)^{*}=p S^{*} p=p^{*} S p={ }^{*} T$.

The following proposition is very important.

Proposition 3.14. Let ${ }_{A} X_{B}$ be a Hilbert $C^{*}$-bimodule of finite type. We assume that $Z(A)=\mathbf{C} \cdot I$ and $Z(B)=\mathbf{C} \cdot I$. Let $\tilde{E}$ be the operator valued weight from $\mathbf{L}_{B}\left(X_{B}\right)$ to $A$ given by a right $B$-bases in $X$, and let $E$ be the conditional expectation from $\mathbf{L}_{B}\left(X_{B}\right)$ to $A$ given by $E(T)=(\mathrm{r}-\operatorname{Ind}[E])^{-1} \tilde{E}(T)$.

If $E$ is minimal, then ${ }_{A} X_{B}$ is semisimple.

Proof. Let $T \in{ }_{A} \operatorname{End}_{B}\left({ }_{A} X_{B}\right)=A^{\prime} \cap \mathbf{L}_{B}\left(X_{B}\right)$ and $x, y \in X$. Put $\Theta_{x, y}(z)=$ $x\langle y, z\rangle_{B}$.

We use the equalities $T \Theta_{x, y}=\Theta_{T x, y}$ and $\Theta_{x, y} T=\Theta_{x, T^{*} y}$ and $\tilde{E}\left(\Theta_{x, y}\right)=$ ${ }_{A}\langle x, y\rangle$. For all $x, y \in X$ we have

$$
\begin{aligned}
{ }_{A}\left\langle x,{ }^{*} T y\right\rangle & ={ }_{A}\langle T x, y\rangle=\tilde{E}\left(\Theta_{T x, y}\right) \\
& =\tilde{E}\left(T \Theta_{x, y}\right)=\tilde{E}\left(\Theta_{x, y} T\right) \\
& =\tilde{E}\left(\Theta_{x, T^{*} y}\right)={ }_{A}\left\langle x, T^{*} y\right\rangle .
\end{aligned}
$$

This shows that $T^{*}={ }^{*} T$.

Lemma 3.15. If $X$ is a minimal Hilbert $A-B$ bimodule, then ${ }_{A} X_{B}$ is semisimple.

Proof. If $X$ is minimal, $E$ in Proposition 3.14 is minimal by Proposition 3.2.

Lemma 3.16. Let $A \subset B$ be a unital inclusion $C^{*}$-algebras and $Z(A)=\mathbf{C} \cdot I$, $Z(B)=\mathbf{C} \cdot I$. Let $E$ be a conditional expectation from $B$ to $A$ of index finite type. Let $X={ }_{B} B_{A}$ be given by $E$. We put $B_{1}=\mathrm{C}^{*}\left\langle B, e_{A}\right\rangle\left(\simeq \mathbf{L}_{A}\left(X_{A}\right)\right)$. Let $F$ be a conditional expectation from $B_{1}$ to $B$ such that $F\left(x e_{A} y\right)=(\operatorname{Index} E)^{-1} x y$.

Then $X \otimes_{A} \bar{X}\left(=B \otimes_{A} B\right)$ and $B_{1}$ are isomorphic as Hilbert $B$ - $B$ bimodules, where $B_{1}$ has a Hilbert $B-B$ bimodule structure induced by $F$. The isomorphism $\varphi$ is given by

$$
\varphi(x \otimes \bar{y})=(\operatorname{Ind}[X])^{1 / 2} x e_{A} y .
$$

Proof. By Lemma 2.2.2 in [47], $B \otimes_{A} B$ and $\mathrm{C}^{*}\left\langle B, e_{A}\right\rangle=B_{1}$ are isomorphic. $\varphi$ is a renormalization of this isomorphism.

We compare two inner products. For $x_{1}, x_{2}, y_{1}, y_{2} \in B$,

$$
{ }_{B}\left\langle x_{1} \otimes y_{1}, x_{2} \otimes y_{2}\right\rangle={ }_{B}\left\langle x_{1},{ }_{A}\left\langle y_{1}, y_{2}\right\rangle, x_{2}\right\rangle=x_{1} E\left(y_{1} y_{2}^{*}\right) x_{2}^{*} .
$$

On the other hand,

$$
\begin{aligned}
{ }_{B}\left\langle x_{1} e_{A} y_{1}, x_{2} e_{A} y_{2}\right\rangle & =F\left(\left(x_{1} e_{A} y_{1}\right)\left(x_{2} e_{A} y_{2}\right)^{*}\right) \\
& =F\left(x_{1} E\left(y_{1} y_{2}^{*}\right) e_{A} x_{2}^{*}\right) \\
& =(\operatorname{Index} E)^{-1} x_{1} E\left(y_{1} y_{2}^{*}\right) x_{2}^{*} .
\end{aligned}
$$

We argue similarly for the right inner product.

Renormalization by $\operatorname{Ind}[E]$ is essentially the same as in the Frobenius reciprocity theorem. 
Lemma 3.17. Let ${ }_{A} X_{B}$ and ${ }_{A} Y_{B}$ be Hilbert $A-B$ bimodules of finite type. We assume that ${ }_{A} X_{B}$ is irreducible, $Z(A)=\mathbf{C} \cdot I$ and $Z(B)=\mathbf{C} \cdot I$. We define a map $\varphi$ from $\left(X \otimes_{B} \bar{Y}\right)^{A}$ to ${ }_{A} \operatorname{Hom}_{B}\left({ }_{A} Y_{B},{ }_{A} X_{B}\right)$ by

$$
\varphi\left(\sum_{i} p_{i} \otimes \overline{q_{i}}\right)=(\mathrm{r}-\operatorname{Ind}[X])^{-1 / 2} \sum_{i} \Theta_{p_{i}, q_{i}} .
$$

Then $\varphi$ gives an isomorphism as Hilbert spaces.

Proof. We calculate inner products. The conclusions follow from the irreducibility and Lemma 3.16.

Proposition 3.18. Let $A \subset B$ be a unital inclusion of $C^{*}$-algebras and $E$ a conditional expectation from $B$ to $A$ of index finite type. We assume that $Z(A)=\mathbf{C} \cdot I$ and $Z(B)=\mathbf{C} \cdot I$. We define the tower of basic construction: $B_{1}=\mathrm{C}^{*}\left\langle B, e_{A}\right\rangle$, $B_{i+1}=\mathrm{C}^{*}\left\langle B_{i}, e_{B_{i-1}}\right\rangle$. We put $X={ }_{B} B_{A}$. Then $B_{n}$ and $B \otimes_{A} B \otimes_{A} \cdots \otimes_{A} B$ (the $n-1$ times tensor of $B$ ) are isomorphic as Hilbert $B$ - $B$ bimodules. For the $n+1$ times tensor $B \otimes_{A} B \otimes_{A} \cdots \otimes_{A} B$, we make this into a Hilbert $B$-B bimodule by the isomorphism between this and the $n$ times tensor product $\left(X \otimes_{A} \bar{X}\right) \otimes_{B} \cdots \otimes_{B}\left(X \otimes_{A} \bar{X}\right)$.

Proof. We use the previous Lemma 3.17 repeatedly:

$$
\begin{aligned}
& B_{1} \simeq B \otimes_{A} B, \\
& B_{2} \simeq B_{1} \otimes_{B} B_{1} \simeq\left(B \otimes_{A} B\right) \otimes_{B}\left(B \otimes_{A} B\right) \simeq B \otimes_{A} B \otimes_{A} B,
\end{aligned}
$$

and so on.

We want to show that the tensor product of minimal bimodules is also minimal. We need some preparation.

Lemma 3.19. Let ${ }_{A} Y_{B}$ be a Hilbert $C^{*}$-bimodule of finite type and ${ }_{B} Z$ a left Hilbert $C^{*}$-module of finite type. For $z \in Z$ we define $R_{z}: Y \rightarrow Y \otimes_{B} Z$ such that $R_{z}(y)=y \otimes z$. Then $R_{z} \in{ }_{A} \operatorname{Hom}\left({ }_{A} Y,{ }_{A}\left(Y \otimes_{B} Z\right)\right)$, and we have

$$
{ }^{*} R_{z}(u \otimes v)=u_{B}\langle v, z\rangle .
$$

Proof. Let $y \in Y$. Then

$$
{ }_{A}\left\langle{ }^{*} R_{z}(u \otimes v), y\right\rangle={ }_{A}\langle u \otimes v, y \otimes z\rangle={ }_{A}\left\langle u_{B}\langle v, z\rangle, y\right\rangle .
$$

Lemma 3.20. Let ${ }_{A} Y_{B}$ be a Hilbert $C^{*}$-bimodule of finite type and $Z={ }_{B} Z_{B}$ a Hilbert $B-B$ bimodule of finite type. If $z \in Z$ is a central element, we have

$$
R_{z}^{*}(u \otimes v)=u\langle z, v\rangle_{B}
$$

Proof. Let $y \in Y$. Then

$$
\begin{aligned}
\left\langle R_{z}^{*}(u \otimes v), y\right\rangle_{B} & =\langle u \otimes v, y \otimes z\rangle_{B}=\left\langle u,\langle u, y\rangle_{B} z\right\rangle_{B} \\
& =\left\langle v, z\langle u, y\rangle_{B}\right\rangle_{B}=\langle v, z\rangle_{B}\langle u, y\rangle_{B} \\
& =\left\langle u\langle z, v\rangle_{B}, y\right\rangle_{B} .
\end{aligned}
$$


We take a right $B$-basis $\left\{u_{i}\right\}_{i}$ in $X$, a left $A$-basis $\left\{v_{j}\right\}_{j}$ in $X$, a right $C$-basis $\left\{t_{l}\right\}_{l}$ in $Y$ and a left $B$-basis $\left\{s_{k}\right\}_{k}$ in $Y$. That is, for $x \in X$ and $y \in Y$,

$$
\begin{array}{ll}
x=\sum_{i} u_{i}\left\langle u_{i}, x\right\rangle_{B}, & x=\sum_{j}{ }_{A}\left\langle x, v_{j}\right\rangle v_{j}, \\
y=\sum_{l} t_{l}\left\langle t_{l}, y\right\rangle_{C}, & y=\sum_{k} B\left\langle y, s_{k}\right\rangle s_{k} .
\end{array}
$$

We define linear maps $\tilde{E}$ and $E$ from $\mathbf{L}_{B}\left(X_{B}\right)$ to $A, \tilde{F}$ and $F$ from $\mathbf{L}_{C}\left(Y_{C}\right)$ to $B$, and $\tilde{H}$ and $H$ from $\mathbf{L}_{C}\left(\left(X \otimes_{B} Y\right)_{C}\right)$ to $A$ as follows.

For $T \in \mathbf{L}_{B}\left(X_{B}\right)$,

$$
\tilde{E}(T)=\sum_{i}{ }_{A}\left\langle T u_{i}, u_{i}\right\rangle, \quad E(T)=(\mathrm{r}-\operatorname{Ind}[X])^{-1} \tilde{E}(T) .
$$

For $T \in \mathbf{L}_{C}\left(Y_{C}\right)$,

$$
\tilde{F}(T)=\sum_{l}\left\langle T t_{l}, t_{l}\right\rangle, \quad F(T)=(\mathrm{r}-\operatorname{Ind}[T])^{-1} \tilde{F}(T) .
$$

For $T \in \mathbf{L}_{C}\left(\left(X \otimes_{B} Y\right)_{C}\right)$,

$$
\tilde{H}(T)=\sum_{i, j}\left\langle T\left(u_{i} \otimes t_{j}\right), u_{i} \otimes t_{j}\right\rangle, \quad H(T)=\left(\mathrm{r}-\operatorname{Ind}\left[X \otimes_{B} Y\right]\right)^{-1} \tilde{H}(T) .
$$

$E, F$ and $H$ are conditional expectations. We define a conditional expectation $G$ from $\mathbf{L}_{C}\left(\left(X \otimes_{B} Y\right)_{C}\right)$ to $\mathbf{L}_{B}\left(X_{B}\right)$.

Definition 3.21. Let ${ }_{A} X_{B}$ and ${ }_{B} Y_{C}$ be Hilbert $\mathrm{C}^{*}$-bimodules of finite type. We take a right $C$-basis $\left\{t_{j}\right\}_{j}$ in $Y$, and put

$$
t=\sum_{i} t_{i} \otimes \bar{t}_{i} \in Y \otimes_{C} \bar{Y}
$$

Then $t$ is a central element in ${ }_{B}\left(Y \otimes_{C} \bar{Y}\right)_{B}$. Using this, we define a bounded operator valued weight $\tilde{G}$. We assume that $Z(B)=\mathbf{C} \cdot I$. We remark that $\langle t, t\rangle_{B}$ is a positive constant.

Definition 3.22. We define linear maps $\tilde{G}$ and $G$ as follows:

$$
\tilde{G}(T)=R_{t}^{*}\left(T \otimes I d_{\bar{Y}}\right) R_{t}, \quad G(T)=(\mathrm{r}-\operatorname{Ind}[Y])^{-1} \tilde{G}(T) .
$$

For $T \in \mathbf{L}_{B}\left(X_{B}\right)$, we put $i(T)=T \otimes I d_{Y} \in \mathbf{L}_{C}\left(\left(X \otimes_{B} Y\right)_{C}\right)$.

Lemma 3.23. $\tilde{G}$ is a positive linear map from $\mathbf{L}_{C}\left(\left(X \otimes_{B} Y\right)_{C}\right)$ to $\mathbf{L}_{B}\left(X_{B}\right)$, and $G$ is a conditional expectation from $\mathbf{L}_{C}\left(\left(X \otimes_{B} Y\right)_{C}\right)$ to $\mathbf{L}_{B}\left(X_{B}\right)$.

Proof. We assume $T \geq 0$. Then

$$
\left\langle R_{t}^{*}\left(\left(T \otimes I d_{\bar{Y}}\right) R_{t}\right) x, x\right\rangle_{B}=\left\langle\left(T \otimes I d_{\bar{Y}}\right) R_{t} x, R_{t} x\right\rangle_{B} \geq 0 .
$$

Since $R_{t}, T \otimes I d_{\bar{Y}}$, and $R_{t}^{*}$ are right $B$-linear, $\tilde{G}(T) \in \mathbf{L}_{B}\left(X_{B}\right)$.

For $S \in \mathbf{L}_{B}\left(X_{B}\right)$,

$$
\begin{aligned}
\tilde{G} i(S) x & =\tilde{G}\left(S \otimes I d_{Y}\right) x=R_{t}^{*}\left(S \otimes I d_{Y} \otimes I d_{\bar{Y}}\right) R_{t} x \\
& =R_{t}^{*}(S x \otimes t)=S x\langle t, t\rangle_{B} \\
& =(\mathrm{r}-\operatorname{Ind}[Y]) S x .
\end{aligned}
$$

Then we have $G(I)=I$, and also $G \geq 0,\|G\|=1$ and $G^{2}=G$. This shows that $G$ is a norm one projection and is a conditional expectation. 
Lemma 3.24. The value of $G$ for rank one operators is as follows. For $x_{1}, x_{2} \in X$ and $y_{1}, y_{2} \in Y$,

$$
G\left(\Theta_{x_{1} \otimes y_{1}, x_{2} \otimes y_{2}}\right)=(\mathrm{r}-\operatorname{Ind}[Y])^{-1} \Theta_{x_{1 B}\left\langle y_{1}, y_{2}\right\rangle, x_{2}} .
$$

Proof. We remark that for $x_{1} \in X, y_{1} \in Y$ and $z_{1} \in Z$

$$
R_{t}^{*}\left(x_{1} \otimes y_{1} \otimes \bar{y}_{2}\right)=x_{1 B}\left\langle y_{1}, y_{2}\right\rangle
$$

We calculate the value of rank one operators:

$$
\begin{aligned}
\tilde{G}\left(\Theta_{x_{1} \otimes y_{1}, x_{2} \otimes y_{2}}\right)(x) & =R_{t}^{*}\left(\Theta_{x_{1} \otimes y_{1}, x_{2} \otimes y_{2}} \otimes I d_{\bar{Y}}\right) R_{t} x \\
& =\sum_{i} R_{t}^{*}\left(x_{1} \otimes y_{1}\left\langle x_{2} \otimes y_{2}, x \otimes t_{i}\right\rangle_{C}\right) \otimes \bar{t}_{i} \\
& =\sum_{i} R_{t}^{*}\left(x_{1} \otimes y_{1}\left\langle y_{2},\left\langle x_{2}, x\right\rangle_{B} t_{i}\right\rangle_{C}\right) \otimes \bar{t}_{i} \\
& =\sum_{i} x_{1 B}\left\langle y_{1}\left\langle y_{2},\left\langle x_{2}, x\right\rangle_{B} t_{i}\right\rangle_{C}, t_{i}\right\rangle \\
& =x_{1 B}\left\langle y_{1}, \sum_{i} t_{i}\left\langle t_{i},\left\langle x_{2}, x\right\rangle_{B}^{*} y_{2}\right\rangle_{C}\right\rangle \\
& =x_{1 B}\left\langle y_{1}, y_{2}\right\rangle\left\langle x_{2}, x\right\rangle_{B} \\
& =\left(\Theta_{x_{1 B}\left\langle y_{1}, y_{2}\right\rangle, x_{2}}\right)(x)
\end{aligned}
$$

By this we have

$$
\tilde{G}\left(\Theta_{x_{1} \otimes y_{1}, x_{2} \otimes y_{2}}\right)=\Theta_{x_{1 B}\left\langle y_{1}, y_{2}\right\rangle, x_{2}} .
$$

Lemma 3.25. $E \circ G=H$.

Proof. We remark that $\mathrm{r}-\operatorname{Ind}\left[X \otimes_{B} Y\right]=(\mathrm{r}-\operatorname{Ind}[X]) \cdot(\mathrm{r}-\operatorname{Ind}[Y])$ because the centers of $A, B$ and $C$ consist of scalars. Then it is sufficient to show that

$$
\tilde{E} \circ \tilde{G}(T)=\sum_{i, j}{ }_{A}\left\langle T\left(u_{i} \otimes t_{j}\right), u_{i} \otimes t_{j}\right\rangle .
$$

We may assume that $T=\Theta_{x_{1} \otimes y_{1}, x_{2} \otimes y_{2}} \in \mathbf{L}_{C}\left(\left(X \otimes_{B} Y\right)_{C}\right)$. Then

$$
\tilde{H}\left(\Theta_{x_{1} \otimes y_{1}, x_{2} \otimes y_{2}}\right)={ }_{A}\left\langle x_{1} \otimes y_{1}, x_{2} \otimes y_{2}\right\rangle .
$$

By Lemma 3.24,

$$
\tilde{E} \circ \tilde{G}\left(\Theta_{x_{1} \otimes y_{1}, x_{2} \otimes y_{2}}\right)=\tilde{E}\left(\Theta_{x_{1 B}\left\langle y_{1}, y_{2}\right\rangle, x_{2}}\right)={ }_{A}\left\langle x_{1 B}\left\langle y_{1}, y_{2}\right\rangle, x_{2}\right\rangle .
$$

The conclusion is only the definition of inner product on tensor products.

Lemma 3.26. The following identity holds:

$$
\left.G\right|_{I d_{X} \otimes_{B} \operatorname{End}_{C}\left({ }_{B} Y_{C}\right)}=\left.F\right|_{B} \operatorname{End}_{C}\left({ }_{B} Y_{C}\right) .
$$


Proof. We take $T \in{ }_{B} \operatorname{End}_{C}\left({ }_{B} Y_{C}\right)$. For $x \in X$,

$$
\begin{aligned}
\tilde{G}\left(I d_{X} \otimes T\right)(x) & =R_{t}^{*}\left(I d_{X} \otimes T \otimes I d_{\bar{Y}}\right) R_{t}(x) \\
& =\sum_{i} R_{t}^{*}\left(I d_{X} \otimes T \otimes I d_{\bar{Y}}\right)\left(x \otimes t_{i} \otimes \bar{t}_{i}\right) \\
& =\sum_{i, j} x\left\langle t_{j} \otimes \bar{t}_{j}, T t_{i} \otimes \bar{t}_{i}\right\rangle_{B} \\
& =\sum_{i, j} x\left\langle\bar{t}_{j},\left\langle t_{j}, T t_{i}\right\rangle_{C} \bar{t}_{i}\right\rangle_{B} \\
& =\sum_{i, j} x_{B}\left\langle t_{j}, t_{i}\left\langle t_{j}, T t_{i}\right\rangle_{C}^{*}\right\rangle \\
& =\sum_{i, j} x_{B}\left\langle t_{j}\left\langle t_{j}, T t_{i}\right\rangle_{C}, t_{i}\right\rangle \\
& =\sum_{i} x_{B}\left\langle\sum_{j} t_{j}\left\langle t_{j}, T t_{i}\right\rangle_{C}, t_{i}\right\rangle \\
& =\sum_{i} x_{B}\left\langle T t_{i}, t_{i}\right\rangle \\
& =x \tilde{F}(T) .
\end{aligned}
$$

Since $\tilde{F}(T) \in Z(B)=\mathbf{C} \cdot I$ for $T \in{ }_{B} \operatorname{End}_{C}\left({ }_{B} Y_{C}\right)$, it follows that

$$
\tilde{G}\left(I d_{X} \otimes T\right)=\tilde{F}(T) \cdot I .
$$

Both sides are scalars.

Next, we construct a quasi basis for the expectation $G$.

Lemma 3.27. We put

$$
P_{i, j, k, l}=\sqrt{1-\operatorname{Ind}[X]} \sqrt{\mathrm{r}-\operatorname{Ind}[Y]} \Theta_{u_{i} \otimes t_{l}, v_{j} \otimes s_{k}} .
$$

Then $\left\{P_{i, j, k, l}, P_{i, j, k, l}^{*}\right\}_{i, j, k, l}$ is a quasi basis for $G$.

Proof. We put $\tilde{P}_{i, j, k, l}=\Theta_{u_{i} \otimes t_{l}, v_{j} \otimes s_{k}}$. For $T \in \mathbf{L}_{C}\left(\left(X \otimes_{B} Y\right)_{C}\right)$, we must show that

$$
T=\sum_{i, j, k, l} P_{i, j, k, l} G\left(P_{i, j, k, l}^{*} T\right) .
$$

We may put $T=\Theta_{x_{1} \otimes y_{1}, x_{2} \otimes y_{2}}$. Then

$$
\begin{aligned}
\tilde{P}_{i, j, k, l}^{*} \Theta_{x_{1} \otimes y_{1}, x_{2} \otimes y_{2}} & =\Theta_{v_{j} \otimes s_{k}, u_{i} \otimes t_{l}} \Theta_{x_{1} \otimes y_{1}, x_{2} \otimes y_{2}} \\
& =\Theta_{v_{j} \otimes s_{k}\left\langle t_{l},\left\langle u_{i}, x_{1}\right\rangle_{B} y_{1}\right\rangle_{C}, x_{2} \otimes y_{2}} .
\end{aligned}
$$


We calculate as follows. Let $x \in X$; then

$$
\begin{aligned}
\tilde{G} & \left(\tilde{P}_{i, j, k, l}^{*} \Theta_{x_{1} \otimes y_{1}, x_{2} \otimes y_{2}}\right) x \\
= & R_{t}^{*}\left(\left(\tilde{P}_{i, j, k, l}^{*} \Theta_{x_{1} \otimes y_{1}, x_{2} \otimes y_{2}}\right) \otimes I d_{\bar{Y}}\right) R_{t} x \\
= & R_{t}^{*}\left(\Theta_{v_{j} \otimes s_{k}\left\langle t_{l},\left\langle u_{i}, x_{1}\right\rangle_{B}\right\rangle_{C}, x_{2} \otimes y_{2}} \otimes I d_{\bar{Y}}\right)\left(\sum_{r} x \otimes t_{r} \otimes \bar{t}_{r}\right) \\
= & \sum_{r} R_{t}^{*}\left(v_{j} \otimes s_{k}\left\langle t_{l},\left\langle u_{i}, x_{1}\right\rangle_{B} y_{1}\right\rangle_{C}\left\langle x_{2} \otimes y_{2}, x \otimes t_{r}\right\rangle_{C} \otimes \bar{t}_{r}\right) \\
= & \sum_{r} R_{t}^{*}\left(v_{j} \otimes s_{k}\left\langle t_{l},\left\langle u_{i}, x_{1}\right\rangle_{B} y_{1}\right\rangle_{C}\left\langle y_{2},\left\langle x_{2}, x\right\rangle_{B} t_{r}\right\rangle_{C} \otimes \bar{t}_{r}\right) \\
= & \sum_{r, q} v_{j}\left\langle t_{q} \otimes \bar{t}_{q}, s_{k}\left\langle t_{l},\left\langle u_{i}, x_{1}\right\rangle_{B} y_{1}\right\rangle_{C}\left\langle y_{2},\left\langle x_{2}, x\right\rangle_{B} t_{r}\right\rangle_{C} \otimes \bar{t}_{r}\right\rangle_{B} \\
= & \sum_{r, q} v_{j}\left\langle\bar{t}_{q},\left\langle t_{q}, s_{k}\left\langle t_{l},\left\langle u_{i}, x_{1}\right\rangle_{B} y_{1}\right\rangle_{C}\left\langle y_{2},\left\langle x_{2}, x\right\rangle_{B} t_{r}\right\rangle_{C}\right\rangle_{C} \bar{t}_{r}\right\rangle_{B} \\
= & \sum_{r, q} v_{j B}\left\langle t_{q}, t_{r}\left[\left\langle t_{q}, s_{k}\right\rangle_{C}\left\langle t_{l},\left\langle u_{i}, x_{1}\right\rangle_{B} y_{1}\right\rangle_{C}\left\langle y_{2},\left\langle x_{2}, x\right\rangle_{B} t_{r}\right\rangle_{C}\right]^{*}\right\rangle \\
= & \sum_{r, q} v_{j B}\left\langle t_{q}\left\langle t_{q}, s_{k}\right\rangle_{C}\left\langle t_{l},\left\langle u_{i}, x_{1}\right\rangle_{B} y_{1}\right\rangle_{C}\left\langle y_{2},\left\langle x_{2}, x\right\rangle_{B}, t_{r}\right\rangle_{C}, t_{r}\right\rangle \\
= & \sum_{r} v_{j B}\left\langle s_{k}\left\langle t_{l},\left\langle u_{i}, x_{1}\right\rangle_{B} y_{1}\right\rangle_{C}\left\langle y_{2},\left\langle x_{2}, x\right\rangle_{B} t_{r}\right\rangle_{C}, t_{r}\right\rangle \\
= & \sum_{r} v_{j B}\left\langle s_{k}\left\langle t_{l},\left\langle u_{i}, x_{1}\right\rangle_{B} y_{1}\right\rangle_{C}, t_{r}\left\langle\left\langle x_{2}, x\right\rangle_{B} t_{r}, y_{2}\right\rangle_{C}\right\rangle \\
= & v_{j B}\left\langle s_{k}\left\langle t_{l},\left\langle u_{i}, x_{1}\right\rangle_{B} y_{1}\right\rangle_{C}, \sum_{r} t_{r}\left\langle t_{r},\left\langle x, x_{2}\right\rangle_{B} y_{2}\right\rangle_{C}\right\rangle \\
= & v_{j B}\left\langle s_{k}\left\langle t_{l},\left\langle u_{i}, x_{1}\right\rangle_{B} y_{1}\right\rangle_{C},\left\langle x, x_{2}\right\rangle_{B} y_{2}\right\rangle .
\end{aligned}
$$

Moreover,

$$
\begin{aligned}
\sum_{i, j, k, l} & \tilde{P}_{i, j, k, l}\left(\tilde{G}\left(\tilde{P}_{i, j, k, l} \Theta_{x_{1} \otimes y_{1}, x_{2} \otimes y_{2}}\right) \otimes I d_{Y}\right)(x \otimes y) \\
= & \sum_{i, j, k, l} \Theta_{u_{i} \otimes t_{l}, v_{j} \otimes s_{k}}\left(\left[v_{j B}\left\langle s_{k}\left\langle t_{l},\left\langle u_{i}, x_{1}\right\rangle_{B} y_{1}\right\rangle_{C},\left\langle x, x_{2}\right\rangle_{B} y_{2}\right\rangle\right] \otimes y\right) \\
= & \sum_{i, j, k, l}\left(u_{i} \otimes t_{l}\right) \\
& \cdot\left\langle s_{k},\left\langle v_{j}, v_{j B}\left\langle s_{k}\left\langle t_{l},\left\langle u_{i}, x_{1}\right\rangle_{B} y_{1}\right\rangle_{C},\left\langle x, x_{2}\right\rangle_{B} y_{2}\right\rangle\right\rangle_{B} y\right\rangle_{C} \\
= & \left(\sum_{j}\left\langle v_{j}, v_{j}\right\rangle_{B}\right) \\
& \cdot \sum_{i, k, l}\left(u_{i} \otimes t_{l}\right)\left\langle s_{k},{ }_{B}\left\langle s_{k}\left\langle t_{l},\left\langle u_{i}, x_{1}\right\rangle_{B} y_{1}\right\rangle_{C}, y_{2}\right\rangle\left\langle x, x_{2}\right\rangle_{B}^{*} y\right\rangle_{C}
\end{aligned}
$$




$$
\begin{aligned}
= & \left(\sum_{j}\left\langle v_{j}, v_{j}\right\rangle_{B}\right) \\
& \cdot \sum_{i, k, l}\left(u_{i} \otimes t_{l}\right)\left\langle{ }_{B}\left\langle y_{2}, s_{k}\left\langle t_{l},\left\langle u_{i}, x_{1}\right\rangle_{B} y_{1}\right\rangle_{C}\right\rangle s_{k},\left\langle x_{2}, x\right\rangle_{B} y\right\rangle_{C} \\
= & \left(\sum_{j}\left\langle v_{j}, v_{j}\right\rangle_{B}\right) \sum_{i, l}\left(u_{i} \otimes t_{l}\right)\left\langle y_{2},\left\langle t_{l},\left\langle u_{i}, x_{1}\right\rangle_{B} y_{1}\right\rangle_{C}^{*},\left\langle x_{2}, x\right\rangle_{B} y\right\rangle_{C} \\
= & \left(\sum_{j}\left\langle v_{j}, v_{j}\right\rangle_{B}\right) \sum_{i, l}\left(u_{i} \otimes t_{l}\right)\left\langle t_{l},\left\langle u_{i}, x_{1}\right\rangle_{B} y_{1}\right\rangle_{C}\left\langle y_{2},\left\langle x_{2}, x\right\rangle_{B} y\right\rangle_{C} \\
= & \left(\sum_{j}\left\langle v_{j}, v_{j}\right\rangle_{B}\right) \sum_{i} u_{i} \otimes\left\langle u_{i}, x_{1}\right\rangle_{B} y_{1}\left\langle y_{2},\left\langle x_{2}, x\right\rangle_{B} y\right\rangle_{C} \\
= & \left(\sum_{j}\left\langle v_{j}, v_{j}\right\rangle_{B}\right)\left(x_{1} \otimes y_{1}\right)\left\langle y_{2},\left\langle x_{2}, x\right\rangle_{B} y\right\rangle_{C} .
\end{aligned}
$$

On the other hand,

$$
\Theta_{x_{1} \otimes y_{1}, x_{2} \otimes y_{1}}(x \otimes y)=\left(x_{1} \otimes y_{1}\right)\left\langle y_{2},\left\langle x_{2}, x\right\rangle_{B} y\right\rangle_{C} .
$$

Identifying $\mathbf{L}_{B}\left(X_{B}\right)$ and $\mathbf{L}_{B}\left(X_{B}\right) \otimes I d_{Y}$, we get

$$
\tilde{P}_{i, j, k, l}\left(\tilde{G}\left(\tilde{P}_{i, j, k, l}^{*} \Theta_{x_{1} \otimes y_{1}, x_{2} \otimes y_{2}}\right)\right)=(\mathrm{l}-\operatorname{Ind}[X]) \Theta_{x_{1} \otimes y_{1}, x_{2} \otimes y_{2}} .
$$

Since $G=(\mathrm{r}-\operatorname{Ind}[Y])^{-1} \tilde{G}$,

$$
\tilde{P}_{i, j, k, l}\left(G\left(\tilde{P}_{i, j, k, l}^{*} \Theta_{x_{1} \otimes y_{1}, x_{2} \otimes y_{2}}\right)\right)=(\mathrm{l}-\operatorname{Ind}[X])(\mathrm{r}-\operatorname{Ind}[Y])\left(\Theta_{x_{1} \otimes y_{1}, x_{2} \otimes y_{2}}\right) .
$$

Then, putting

$$
P_{i, j, k, l}=\sqrt{\mathrm{l}-\operatorname{Ind}[X]} \sqrt{\mathrm{r}-\operatorname{Ind}[Y]} \tilde{P}_{i, j, k, l},
$$

we see that $\left\{P_{i, j, k, l}, P_{i, j, k, l}^{*}\right\}_{i, j, k, l}$ is a quasi basis for $G$.

Lemma 3.28. $G: \mathbf{L}_{C}\left(\left(X \otimes_{B} Y\right)_{C}\right) \rightarrow \mathbf{L}_{B}\left(X_{B}\right)$ is minimal.

Proof. We have $\left(\mathbf{L}_{B}\left(X_{B}\right) \otimes I d_{Y}\right)^{\prime} \cap \mathbf{L}_{C}\left(\left(X \otimes_{B} Y\right)_{C}\right)=I d_{X} \otimes_{B} \operatorname{End}_{C}\left({ }_{B} Y_{C}\right)$ from Lemma 1.25 in chapter 1 . We take $T \in{ }_{B} \operatorname{End}_{C}\left({ }_{B} Y_{C}\right)$.

By Lemma 3.27, we have $\tilde{G}\left(I d_{X} \otimes T\right)=\tilde{F}(T)$. On the other hand,

$$
\begin{aligned}
\sum_{i, j, k, l} & \Theta_{u_{i} \otimes t_{l}, v_{j} \otimes s_{k}}\left(I d_{X} \otimes T\right) \Theta_{u_{i} \otimes t_{l}, v_{j} \otimes s_{k}}^{*} \\
= & \sum_{i, j, k, l} \Theta_{u_{i} \otimes t_{l}, v_{j} \otimes s_{k}} \Theta_{v_{j} \otimes T s_{k}, u_{i} \otimes t_{l}} \\
= & \sum_{i, j, k, l} \Theta_{u_{i} \otimes t_{l}\left\langle s_{k},\left\langle v_{j}, v_{j}\right\rangle_{B} T s_{k}\right\rangle_{C}, u_{i} \otimes t_{l}} \\
= & \left(\sum_{j}\left\langle v_{j}, v_{j}\right\rangle_{B}\right) \sum_{i, l} \Theta_{u_{i} \otimes t_{l}\left(\sum_{k}\left\langle s_{k}, T s_{k}\right\rangle_{C}\right), u_{i} \otimes t_{l}} \\
= & \left(\sum_{j}\left\langle v_{j}, v_{j}\right\rangle_{B}\right) \sum_{k}\left\langle s_{k}, T s_{k}\right\rangle_{C} \sum_{i, l} \Theta_{u_{i} \otimes t_{l}, u_{i} \otimes t_{l}} \\
= & \sum_{j}\left\langle v_{j}, v_{j}\right\rangle_{B} \sum_{k}\left\langle s_{k}, T s_{k}\right\rangle_{C} .
\end{aligned}
$$


We have used the fact that $T \in{ }_{B} \operatorname{End}_{C}\left({ }_{B} Y_{C}\right)$. Since ${ }_{B} Y_{C}$ is a minimal bimodule, the last term is equal to

$$
\text { (const) } \sum_{j}\left\langle v_{j}, v_{j}\right\rangle_{B} \sum_{l} B\left\langle T t_{l}, t_{l}\right\rangle=(\text { const }) \tilde{F}(T)
$$

Theorem 3.29. Let $A, B$ and $C$ be unital $C^{*}$-algebras whose centers consist of scalars, and let $X={ }_{A} X_{B}$ and $Y={ }_{B} Y_{C}$ be Hilbert $C^{*}$-bimodules of finite type. If $X$ and $Y$ are minimal bimodule, then $X \otimes_{B} Y$ is also minimal.

Proof. To finish the proof of the theorem, it is sufficient to show that $H$ is a minimal expectation. But we have $H=E \circ G$. Since $G$ and $E$ are both minimal, $H$ is also a minimal expectation by Theorem 3 in 28 .

Corollary 3.30. If ${ }_{A} X_{B}$ and ${ }_{B} Y_{C}$ are semisimple, ${ }_{A}\left(X \otimes_{B} Y\right)_{C}$ is also semisimple.

Proof. $X$ and $Y$ are direct sums of irreducible bimodules. Since irreducible bimodules are minimal, a tensor product of two irreducible bimodules is minimal, and semisimple.

Theorem 3.31. Let ${ }_{A} X_{B}$ and ${ }_{B} Y_{A}$ be Hilbert $C^{*}$-bimodules of finite type. We assume that ${ }_{A} X_{B}$ and ${ }_{B} Y_{A}$ are irreducible and normalized. In this case the following are equivalent.

(1) ${ }_{B} Y_{A}$ and ${ }_{A} \bar{X}_{B}$ are unitarily equivalent.

(2) $X \otimes_{B} Y$ contains a direct summand which is unitarily equivalent to ${ }_{A} A_{A}$.

(3) $Y \otimes_{A} X$ contains a direct summand which is unitarily equivalent to ${ }_{B} B_{B}$.

Moreover, if one of these conditions is satisfied, then $X \otimes_{B} Y$ contains ${ }_{A} A_{A}$ and $Y \otimes_{A} X$ contains ${ }_{B} B_{B}$, both with multiplicity one.

Proof. By Frobenius reciprocity, ${ }_{B} \operatorname{Hom}_{A}\left({ }_{B} Y_{A},{ }_{B} \bar{X}_{A}\right)$ and $\operatorname{Hom}\left({ }_{A}\left(X \otimes_{B} Y\right)_{A},{ }_{A} A_{A}\right)$ are isomorphic. If irreducible bimodules are similar, by Theorem 2.4 they are necessarily unitarily equivalent. These facts prove this theorem.

\section{Categorical structure}

In this section we shall study several categorical structures of Hilbert $\mathrm{C}^{*}$-bimodules of finite type. In this paper, the terminology tensor category is used for what was called a labeled tensor category in 49 .

Let ${ }_{A} X_{B}$ be a Hilbert $\mathrm{C}^{*}$-bimodule of finite type. Define $\varepsilon_{X}: X \otimes_{B} \bar{X} \rightarrow A$ by

$$
\varepsilon_{X}(p \otimes \bar{q})={ }_{A}\langle p, q\rangle
$$

and $\eta_{X}: B \rightarrow \bar{X} \otimes_{A} X$ by

$$
\eta_{X}(b)=\sum_{j} b\left(\bar{v}_{j} \otimes v_{j}\right)
$$

where $\left\{v_{1}, \ldots, v_{m}\right\}$ is a left $A$-basis for ${ }_{A} X_{B}$ and $\eta_{X}$ dose not depend on the choice of a left $A$-basis. In fact, since there exists a $B$ - $B$ isomorphism $\varphi^{\prime}: \bar{X} \otimes{ }_{A} X \rightarrow{ }_{A} \mathbf{L}\left({ }_{A} X\right)$ with $\varphi^{\prime}(\bar{p} \otimes q)(x)={ }_{A}\langle x, p\rangle q$ by Proposition $2.5, \varphi^{\prime-1}\left(I d_{X}\right)=\sum_{j} v_{j} \otimes v_{j}$ does not depend on the choice of a left $A$-basis of $X$. Moreover, we have

$$
\sum_{j} b\left(\bar{v}_{j} \otimes v_{j}\right)=b \cdot \varphi^{\prime-1}\left(I d_{X}\right)=\varphi^{\prime-1}\left(b \cdot I d_{X}\right)=\varphi^{\prime-1}\left(I d_{X} b\right)=\sum_{j}\left(\bar{v}_{j} \otimes v_{j}\right) b .
$$


It is clear that $\varepsilon_{X}$ and $\eta_{X}$ are bimodule maps. Furthermore, we have that $\varepsilon_{X}^{*}=$ ${ }^{*} \varepsilon_{X}=\eta_{\bar{X}}$, and $\eta_{\bar{X}}: A \rightarrow X \otimes_{B} \bar{X}$ is given by

$$
\eta_{\bar{X}}(a)=\sum_{i} a\left(u_{i} \otimes \bar{u}_{i}\right)=\sum_{i}\left(u_{i} \otimes \bar{u}_{i}\right) a,
$$

where $\left\{u_{i}, \ldots, u_{n}\right\}$ is a right $B$-basis of ${ }_{A} X_{B}$. We shall show that $\left(\bar{X}, \varepsilon_{X}, \eta_{X}\right)$ is a (right) dual of $X$ in the categorical sense (see [25]).

Proposition 4.1. The category of Hilbert $C^{*}$-bimodules of finite type as objects and bimodule maps as arrows with the above $\left(\varepsilon_{X}, \eta_{X}\right)$ is a rigid category in the sense that, for any Hilbert $C^{*}$-bimodules ${ }_{A} X_{B}$ and ${ }_{B} Y_{C}$ of finite type, the following identities hold:

$$
\begin{aligned}
& \text { (1) } \quad\left(\varepsilon_{X} \otimes I d_{X}\right)\left(I d_{X} \otimes \eta_{X}\right)=I d_{X}, \\
& \text { (2) } \quad\left(I d_{\bar{X}} \otimes \varepsilon_{X}\right)\left(\eta_{X} \otimes I d_{\bar{X}}\right)=I d_{\bar{X}}, \\
& \text { (3) } \varepsilon_{X \otimes_{B} Y}=\varepsilon_{X} \circ\left(I d_{X} \otimes \varepsilon_{Y} \otimes I d_{\bar{X}}\right) .
\end{aligned}
$$

Proof. (1) We have

$$
\begin{aligned}
\left(\varepsilon_{X} \otimes I d_{X}\right)\left(I d_{X} \otimes \eta_{X}\right)(x) & =\left(\varepsilon_{X} \otimes I d_{X}\right)\left(\sum_{j} x \otimes \bar{v}_{j} \otimes v_{j}\right) \\
& =\sum_{j}{ }_{A}\left\langle x, v_{j}\right\rangle v_{j}=x .
\end{aligned}
$$

The proof of (2) is similar to that of (1). (3) is only the reformulation of the definition of inner product of tensor product.

Recall that a category $\mathcal{A}$ is called a $\mathrm{C}^{*}$-category if each set of arrows is a Banach space where the composition of arrows gives us a bilinear map $S \times T \rightarrow S \circ T$ with $\|S \circ T\| \leq\|S\|\|T\|$ and there is an antilinear involutive covariant functor $*: \mathcal{A} \rightarrow \mathcal{A}$ such that $\left\|T^{*} \circ T\right\|=\|T\|^{2}$ for each arrow $T$. In the following proposition, we only consider $\mathrm{C}^{*}$-algebras with trivial center.

Proposition 4.2. The tensor category with minimal Hilbert $C^{*}$-bimodules of finite type as objects and bimodule maps as arrows is a $C^{*}$-category by setting $\|T\|=$ $\left\|T^{*} T\right\|^{1 / 2}$ for $T \in{ }_{A} \operatorname{Hom}_{B}(X, Y)$.

Proof. Since any minimal Hilbert $\mathrm{C}^{*}$-bimodules ${ }_{A} X_{B}$ is semisimple by Proposition $3.15,{ }_{A} \operatorname{End}_{B}\left({ }_{A} X_{B}\right)$ is a $\mathrm{C}^{*}$-algebra. Then we can apply the argument in [17, p. $82]$.

Remark. We can replace "minimal" by "semisimple".

The class of minimal Hilbert $\mathrm{C}^{*}$-bimodule of finite type is not closed under direct sum, but the class of normalized ones is closed by Lemma 3.10. Therefore we have the following nice class. Consider unital $\mathrm{C}^{*}$-algebras with trivial centers.

Corollary 4.3. The category with normalized minimal Hilbert $C^{*}$-bimodules of finite type as objects and bimodule maps as arrows is a $C^{*}$-category.

S. Yamagami introduced a metricial structures in tensor category, called an $\varepsilon$ structure, which plays a significant role in his study [49], [50]. The notion of $\varepsilon$-structure has a close relation with rigidity of the category. 
Proposition 4.4. The category of Hilbert $C^{*}$-bimodules of finite type admits the canonical $\varepsilon$-structures defined by $\varepsilon_{X}(p \otimes \bar{q})={ }_{A}\langle p, q\rangle$ for $p, q \in{ }_{A} X_{B}$. That is, the following identities hold for any Hilbert $C^{*}$-bimodules ${ }_{A} X_{B}$ and ${ }_{B} Y_{C}$ of finite type:

(1) $\epsilon_{X \otimes Y}=\epsilon_{X} \circ\left(1_{X} \otimes_{B} \epsilon_{Y} \otimes 1_{\bar{X}}\right)$.

(2) For $T \in{ }_{A} \operatorname{Hom}_{B}\left({ }_{A} X_{B},{ }_{A} Y_{B}\right)$ we have $\epsilon_{X}\left(1_{X} \otimes{ }^{t} T\right)=\epsilon_{Y}\left(T \otimes 1_{\bar{Y}}\right)$.

(3) For $T \in{ }_{A} \operatorname{Hom}_{B}\left({ }_{A} X_{B},{ }_{A} Y_{B}\right), \epsilon_{Y}\left(T \otimes 1_{\bar{Y}}\right)=0$ implies $T=0$.

Proof. (1) is the same as in Proposition 4.1(1). We show (2):

$$
\begin{aligned}
\epsilon_{X}\left(1_{X} \otimes{ }^{t} T\right)(x \otimes \bar{y}) & =\epsilon_{X}\left(x \otimes \overline{{ }^{*} T} \bar{y}\right), \\
& ={ }_{A}\left\langle x,{ }^{*} T y\right\rangle={ }_{A}\langle T x, y\rangle, \\
\epsilon_{Y}\left(T \otimes 1_{\bar{Y}}\right)(x \otimes \bar{y}) & ={ }_{A}\langle T x, y\rangle .
\end{aligned}
$$

We show (3). We assume $\epsilon_{Y}\left(T \otimes 1_{\bar{Y}}\right)=0$. For every $x \in X$ and $y \in Y$, the following identity holds:

$$
\epsilon_{Y}\left(T \otimes 1_{\bar{Y}}\right)(x \otimes \bar{y})=\epsilon_{Y}(T x \otimes \bar{y})={ }_{A}\langle T x, y\rangle=0 .
$$

We can describe the correspondence of Frobenius reciprocity maps explicitly by inner products (cf. [48] and [25]).

Proposition 4.5. Let ${ }_{A} X_{B},{ }_{B} Y_{C}$ and ${ }_{A} Z_{C}$ be Hilbert $C^{*}$-bimodules of finite type.

(1) For $T \in{ }_{A} \operatorname{Hom}_{C}\left({ }_{A} Z_{C},{ }_{A}\left(X \otimes_{B} Y\right)_{C}\right)$,

$$
\left(1_{Y} \otimes \epsilon_{Y}\right)\left(T \otimes 1_{\bar{Y}}\right) \in{ }_{A} \operatorname{Hom}_{C}\left({ }_{A}\left(Z \otimes_{C} \bar{Y}\right)_{B},{ }_{A} X_{B}\right)
$$

is the element corresponding by Frobenius reciprocity. Conversely, for $S \in$ $\left.{ }_{A} \operatorname{Hom}_{B}{ }_{A}\left(Z \otimes_{C} \bar{Y}\right)_{B},{ }_{A} X_{B}\right),\left(S \otimes I d_{Y}\right)\left(I d_{Z} \otimes \varepsilon_{\bar{Y}}^{*}\right)$ is the element corresponding by the inverse of Frobenius reciprocity.

(2) For $T \in{ }_{A} \operatorname{Hom}_{C}\left({ }_{A} Z_{C},{ }_{A}\left(X \otimes_{B} Y\right)_{C}\right)$, the element

$$
\left(\epsilon_{\bar{X}} \otimes 1_{Y}\right)\left(1_{\bar{X}} \otimes T\right) \in{ }_{A} \operatorname{Hom}_{C}\left({ }_{B}\left(\bar{X} \otimes_{A} Z\right)_{C},{ }_{B} Y_{C}\right)
$$

is the element corresponding by Frobenius reciprocity. Conversely, for $S \in$ ${ }_{A} \operatorname{Hom}_{C}\left({ }_{A} \operatorname{Hom}_{C}\left({ }_{B}\left(\bar{X} \otimes_{A} Z\right){ }_{C},{ }_{B} Y_{C}\right),\left(I d_{X} \otimes S\right)\left(\varepsilon_{X} \otimes I d_{Z}\right)\right.$ is the element corresponding by the inverse of Frobenius reciprocity.

Proof. Since all bimodules are of finite type, it is sufficient to prove the above statement for rank one operators.

We show (1). We put $T=\Theta_{x \otimes y, z}$. By Frobenius reciprocity, this operator is transformed into $\mathcal{F}(T)=\Theta_{x, z \otimes \bar{y}}$. We have

$$
\mathcal{F}(T)\left(z^{\prime} \otimes \bar{y}^{\prime}\right)=x\left\langle z \otimes \bar{y}, z^{\prime} \otimes \bar{y}^{\prime}\right\rangle_{B}=x\left\langle\bar{y},\left\langle z, z^{\prime}\right\rangle_{C} \bar{y}^{\prime}\right\rangle_{B}=x_{B}\left\langle y, y^{\prime}\left\langle z^{\prime}, z\right\rangle_{C}\right\rangle .
$$

On the other hand,

$$
\begin{aligned}
\left(1_{X} \otimes \epsilon_{Y}\right)\left(\left(T \otimes 1_{\bar{Y}}\right)\left(z^{\prime} \otimes \bar{y}^{\prime}\right)\right) & =\left(1_{X} \otimes \epsilon_{Y}\right)(x \otimes y)\left\langle z, z^{\prime}\right\rangle_{C} \otimes \bar{y}^{\prime} \\
& =x \otimes{ }_{B}\left\langle y\left\langle z, z^{\prime}\right\rangle_{C}, y^{\prime}\right\rangle .
\end{aligned}
$$

We put $S=\Theta_{x, z \otimes \bar{y}}$. By inverse Frobenius reciprocity, this operator is transformed into $\tilde{\mathcal{F}}(T)=\Theta_{x \otimes y, z}$. We have

$$
\tilde{\mathcal{F}}(T)(z)=(x \otimes y)\left\langle z, z^{\prime}\right\rangle_{C} .
$$


We take a right $C$-basis $\left\{u_{i}\right\}_{i}$ in $Y$. On the other hand,

$$
\begin{aligned}
\left(S \otimes I d_{Y}\right)\left(I d_{Z} \otimes \varepsilon_{\bar{Y}}^{*}\right)\left(z^{\prime}\right) & =\left(S \otimes I d_{Y}\right)\left(z^{\prime} \otimes \sum_{i}\left(\bar{u}_{i} \otimes u_{i}\right)\right) \\
& =\sum_{i} x\left\langle z \otimes \bar{y}, z^{\prime} \otimes \bar{u}_{i}\right\rangle_{B} \otimes u_{i} \\
& =\sum_{i} x\left\langle\bar{y},\left\langle z, z^{\prime}\right\rangle_{C} \bar{u}_{i}\right\rangle_{B} \otimes u_{i} \\
& =\sum_{i} x_{B}\left\langle y, u_{i}\left\langle z^{\prime}, z\right\rangle_{C}\right\rangle \otimes u_{i} \\
& =\sum_{i} x \otimes\left\langle y\left\langle z, z^{\prime}\right\rangle_{C}, u_{i}\right\rangle u_{i} \\
& =(x \otimes y)\left\langle z, z^{\prime}\right\rangle_{C} .
\end{aligned}
$$

We can prove the other parts similarly.

We investigate the categorical structure of Hilbert $\mathrm{C}^{*}$-bimodules given by bundle construction in chapter 1. Let $G, H$ and $K$ be as in chapter 1. Let $\alpha$ be an action of $G$ on a unital $\mathrm{C}^{*}$-algebra, and assume that $\alpha$ is properly outer, i.e. if $\alpha_{g}(x) y=y x$ for every $x \in A$, then $y=0$. For $V={ }_{H} V_{K}$ and $W={ }_{H} W_{K}$, let ${ }_{H} \operatorname{Hom}_{K}(V, W)$ be the set of linear maps from $V$ to $W$ such that $T V_{g} \subset W_{g}$ for each $g \in G$ and $T(h v k)=h(T v) k$ for $h \in H, k \in K$ and $v \in V$. For $T \in{ }_{H} \operatorname{Hom}_{K}(V, W)$, we define $\hat{T}$ by $\hat{T}(a \otimes v)=a \otimes T v$. Then $\hat{T} \in{ }_{A \rtimes H} \operatorname{Hom}_{A \rtimes K}(\hat{V}, \hat{W})$ in the bimodule sense. Then, as in [33, the following proposition holds.

Proposition 4.6. The following hold.

(1) $(V \oplus W) \simeq \hat{V} \oplus \hat{W}$.

(2) $(V \otimes W) \simeq \hat{V} \otimes \hat{W}$.

(3) $\hat{\bar{V}} \simeq \overline{\hat{V}}$.

(4) $B y T \rightarrow \hat{T},{ }_{H} \operatorname{Hom}_{K}(V, W) \simeq{ }_{A \rtimes H} \operatorname{Hom}_{A \rtimes K}(\hat{V}, \hat{W})$.

Proof. The proofs of (1), (2) and (3) are similar to those in [33]. As in [33], we must use the assumption that $\alpha$ is properly outer for the proof of (4). We omit the details.

By the above proposition, the correspondence from a bundle ${ }_{H} V_{K}$ to a bimodule $A \rtimes_{\alpha} H \hat{V}_{A \rtimes_{\alpha} K}$ is considered to be a functor. Moreover, by (4) this functor is an isomorphism. We may compute fusion rules of these bimodules using the computation of bundles as in the von Neumann algebra setting [33].

\section{5. $K K$-THEORY AND $\mathrm{C}^{*}$-INDEX THEORY}

Let $A$ and $B$ be unital $\mathrm{C}^{*}$-algebras, $X={ }_{A} X_{B}$ a Hilbert $\mathrm{C}^{*}$-bimodule of finite type. We can associate an element in $K K(A, B)$ and in $K K(B, A)$, which are called Kasparov $K K$-groups.

Definition 5.1 [1]. Let $A$ and $B$ be two $\mathrm{C}^{*}$-algebras. We call a triple $(\pi, X, F)$ a Kasparov bimodule if

(1) $X$ is a $\mathbf{Z}_{2}$-graded right Hilbert B-module,

(2) $\pi$ is a representation of $\mathrm{A}$ in $\mathbf{L}_{B}\left(X_{B}\right)$ of degree 0 ,

(3) $F$ is a bounded linear operator on $X$ which is of degree 1 , and 
(4) $\pi$ and $F$ satisfy the following conditions:

$$
\pi(a) F-F \pi(a) \in \mathbf{K}_{B}\left(X_{B}\right) \forall a \in A, \quad F^{2}-I \in \mathbf{K}_{B}\left(X_{B}\right), \quad F-F^{*} \in \mathbf{K}_{B}\left(X_{B}\right) .
$$

By taking the quotient by some appropriate equivalence relation, we get the Kasparov $K K$-group $K K(A, B)$ ].

Let $A, B$ and $C$ be three $\mathrm{C}^{*}$-algebras. Then there exists an associative product operation from $K K(A, B) \times K K(B, C)$ to $K K(A, C)$. This is called the Kasparov product. We write $x \otimes y$ for the Kasparov product of $x \in K K(A, B)$ and $y \in$ $K K(B, C)$.

The groups $K_{0}$ and $K_{1}$ are considered as special cases of $K K$-groups. $K_{0}(A)$ is identified to $K K(\mathbf{C}, A) . K_{1}(A)$ is identified to $K K\left(\mathbf{C}, A \otimes C_{0}(R)\right)$. The tensor product algebra $A \otimes C_{0}(R)$ is called the suspension $\mathrm{C}^{*}$-algebra of $A$.

Let $i$ be 0 or 1 . By these identifications, some element $x$ of $K K(A, B)$ gives a map from $K_{i}(A)$ to $K_{i}(B)$ by Kasparov product and Bott periodicity of $K K$-groups. Under certain conditions, $K K(A, B)$ is recovered from

$$
\operatorname{Hom}\left(K_{0}(A), K_{0}(B)\right) \oplus \operatorname{Hom}\left(K_{1}(A), K_{1}(B)\right) .
$$

Lemma 5.2 (Universal coefficient formula [1]). If $K_{i}(A)$ is free or $K_{i}(B)$ is divisible for $i=0,1$ and $A$ is in a certain bootstrap category as in [1], then $K K(A, B)$ and $\operatorname{Hom}\left(K_{0}(A), K_{0}(B)\right) \oplus \operatorname{Hom}\left(K_{1}(A), K_{1}(B)\right)$ are canonically isomorphic.

Let $B$ be a unital $\mathrm{C}^{*}$-algebra, and $A$ be a sub-C*-algebra of $B$ containing the unit of $B$. First we assume that there exists a conditional expectation $E$ from $B$ to $A$ such that $(A \subset B, E)$ is of index finite type.

By the inclusion map from $A$ to $B$, we can define a homomorphism from $K_{i}(A)$ to $K_{i}(B) \quad(i=0,1)$. In [47], we defined the transfer map from $K_{0}(B)$ to $K_{0}(A)$.

In this paper, we define a bimodule of $K K(A, B)$, and describe the transfer map by Kasparov product. We state this construction in the formulation of Hilbert $\mathrm{C}^{*}$-bimodules.

Let $X={ }_{A} X_{B}$ be a Hilbert $A-B$ bimodule, and assume that $X$ is of finite type in the sense of a right $B$-module. We may consider $X$ as a trivially graded right Hilbert B-module with the left representation $\pi$ of $A$ on it. Then as following lemma shows, we can define some Kasparov bimodule canonically.

Lemma 5.3. $v=(\pi, X, 0)$ is a Kasparov element and gives an element of $K K(A, B)$.

We remark that in the situation of inclusion $(A \subset B, E), v$ is always a Kasparov element whether $(A \subset B, E)$ is of index finite type or not, because $B_{B}$ is always of finite type.

We have the conjugate bimodule ${ }_{B} \bar{X}_{A}$ of ${ }_{A} X_{B}$. We denote by $\tilde{\pi}$ the left representation of B on $\bar{X}$.

Lemma 5.4. If $(A \subset B, E)$ is of index finite type, then $u=(\tilde{\pi}, \bar{X}, 0)$ is also a Kasparov element and gives an element of $K K(B, A)$.

We define two $K K$-indices of this inclusion by Kasparov product.

Definition 5.5. Let $X={ }_{A} X_{B}$. For this bimodule, we have $u \in K K(B, A)$ and $v \in K K(A, B)$. Then we call $u \otimes v \in K K(B, B)$ and $v \otimes u \in K K(A, A)$ the $K K$-index of $X$. 
Remark. In many case, $K K$-indices are given by reasonable scalars. In the $I I_{1}$ factor setting, $K K(A, A)$ and $K K(B, B)$ can be interpreted as the automorphism of the additive group $\mathbf{R}$. The $K K$-index is a positive scalar constant in this case (cf. [15]).

We give an example which shows that $K_{1}$ groups is also important in $K K$-index theory.

Example. Let $\mathbf{T}$ be a one dimensional torus and $\tilde{\mathbf{T}}$ a two covering of $\mathbf{T}$. There exists an action $\alpha$ of $\mathbf{Z}_{2}$ on $\tilde{\mathbf{T}}$ such that $\mathbf{T}$ is the quotient space. Let $A=C(\mathbf{T})$ and $B=C(\tilde{\mathbf{T}})$. Let $\beta$ be the action of $\mathbf{Z}_{2}$ on $B$ induced by $\alpha$. Then $A$ is the fixed point subalgebra under $\beta$. The $\mathrm{C}^{*}$-basic extension is the $\mathrm{C}^{*}$-crossed product $B \rtimes \mathbf{Z}_{2}$, which is isomorphic to $A \otimes M_{2}(\mathbf{C})$.

$K_{0}(A)$ is isomorphic to $\mathbf{Z}$, and the generator is the identity $I$. Its image in $B$ is the generator. Its image in $A \otimes M_{2}(\mathbf{C})$ is also the identity. But the identity in $A \otimes M_{2}(\mathbf{C})$ is a 2 times generator in $K_{0}\left(A \otimes M_{2}(\mathbf{C})\right) \simeq K_{0}(A)$. On the other hand, $K_{1}(A)$ is isomorphic to $\mathbf{Z}$ and its generator is $f(z)=z$. Its image in $B$ is $g(z)=z^{2}$ and is a 2 times generator in $K_{1}(B)$. Its image in $A \otimes M_{2}(\mathbf{C})$ is the diagonal matrix $\operatorname{diag}(f, f)$ and this is also a 2-times generator in $K_{1}\left(A \otimes M_{2}(\mathbf{C})\right) \simeq K_{1}(A)$.

This shows that $v=(1,2) \in K K(A, B)$ and $u=(2,1) \in K K(B, A)$. $K K$-indices are as follows: $u \otimes v=2 \cdot I$ and $v \otimes u=2 \cdot I$.

More general cases of circle algebras are studied by Deaconu in [7]. He points out that $n$-folded covering maps and $n$-times around embeddings are dual to each other.

We show that the dimension map is a character on some subring on $K K(A, A)$. We assume that all $\mathrm{C}^{*}$-algebras are unital and separable. We denote by $\gamma_{\alpha}$ the element in $\operatorname{Hom}\left(K_{0}(A), K_{0}(A)\right)$ corresponding to an element $\alpha$ in $K K(A, A)$. We assume that $A$ has a normalized positive trace $\tau$. We denote by $\hat{\tau}$ the trace on $K_{0}(A)$ induced by $\tau$.

Definition 5.6. We define a map $d_{\tau}$ from $K K(A, A)$ to $\mathbf{R}$ by $d_{\tau}(\alpha)=\hat{\tau}\left(\gamma_{\alpha}[1]\right)$. We call this $d_{\tau}(\alpha)$ the dimension of $\alpha \in K K(A, A)$ with respect to $\tau$.

Definition 5.7. We say that a Hilbert $\mathrm{C}^{*}$-bimodule $X={ }_{A} X_{A}$ is compatible with a trace $\tau$ if $\tau\left({ }_{A}\langle x, y\rangle\right)=\tau\left(\langle y, x\rangle_{A}\right)$ for $x, y \in X$.

Example. Let $B$ be a $\mathrm{C}^{*}$-algebra with a normalized trace $\tau$, and let $A$ be a $\mathrm{C}^{*}$ subalgebra of $B$ containing the unit. Let $E$ be the $\tau$-preserving conditional expectation from $B$ to $A$. Let $Y={ }_{A} B_{B}$ be the example after Definition 1.6 and $X=Y \otimes \bar{Y}$. Then $X$ is $\tau$-compatible.

We assume that $Z(A)=\mathbf{C} \cdot I$. For a Hilbert $\mathrm{C}^{*}$-bimodule $X={ }_{A} X_{A}$ of finite type, we put $d(X)=\sqrt{\operatorname{Ind}[X]}$ and call this the dimension of $X$, and we denote by $\varphi(X)$ the corresponding element in $K K(A, A)$.

Proposition 5.8. Let $A$ be a unital $C^{*}$-algebra such that $Z(A)=\mathbf{C} \cdot I, \tau$ a normalized trace on $A$, and $X={ }_{A} X_{A}$ a normalized Hilbert $A$ - $A$ bimodule of finite type which is $\tau$-compatible. We put $f: K_{0}(A) \rightarrow K_{0}(A), f([\mathcal{E}])=\left[\left(\mathcal{E} \otimes_{A} X\right)_{A}\right]$, and 
$V: \mathbf{R} \rightarrow \mathbf{R}, V(t)=d(X) t$. Then the following diagram commutes:

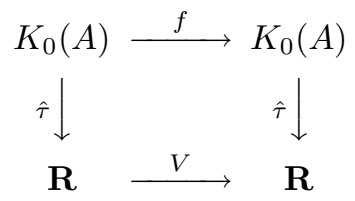

Proof. Let $\mathcal{E}=\mathcal{E}_{A}$ be a Hilbert right $A$-module of finite type, and $\left\{u_{i}\right\}_{i}$ a right $A$ basis in $\mathcal{E}$. Let $p=\left(p_{i, j}\right)_{i, j}=\left(\left\langle u_{i}, u_{j}\right\rangle_{A}\right)_{i, j} \in A \otimes M_{n}(\mathbf{C})$ be a projection such that $\mathcal{E}=p A^{n}$. Let $\left\{s_{j}\right\}_{j}$ be a right $A$-basis in ${ }_{A} X_{A}$ (cf. Lemma 1.11). Then $\left\{u_{i} \otimes s_{j}\right\}_{i, j}$ is a right $A$-basis in $\mathcal{E}_{A} \otimes{ }_{A} X_{A}$. We put $q=\left(\left\langle u_{i} \otimes s_{j}, u_{k} \otimes s_{l}\right\rangle_{A}\right)_{(i, j),(k, l)} \in A \otimes M_{n m}(\mathbf{C})$. Then $\mathcal{E} \otimes_{A} X_{A} \simeq q A^{n m}$, and $\left[\left(\mathcal{E} \otimes_{A} X\right)_{A}\right]=[q] \in K_{0}(A)$.

We have

$$
\hat{\tau}\left(\left[\mathcal{E}_{A}\right]\right)=\hat{\tau}([p])=(\tau \otimes i d)(p)=\sum_{i=1}^{n} \tau\left(\left\langle u_{i}, u_{i}\right\rangle_{A}\right) .
$$

Using these, we have

$$
\begin{aligned}
\hat{\tau}\left(\gamma_{[X]}(\mathcal{E})\right) & =\hat{\tau}\left(\left[\left(\mathcal{E} \otimes_{A} X\right)_{A}\right]\right) \\
& =\hat{\tau}([q]) \\
& =(\tau \otimes I d)(\mathcal{E}) \\
& =\sum_{i, j} \tau\left(\left\langle u_{i} \otimes s_{j}, u_{i} \otimes s_{j}\right\rangle_{A}\right) \\
& =\sum_{i, j} \tau\left(\left\langle\left\langle u_{i}, u_{i}\right\rangle_{A} s_{j}, s_{j}\right\rangle_{A}\right) \\
& =\sum_{i, j} \tau\left({ }_{A}\left\langle s_{j},\left\langle u_{i}, u_{i}\right\rangle_{A} s_{j}\right\rangle\right) \\
& =\sum_{i, j} \tau\left(\left\langle u_{i}, u_{i}\right\rangle_{A A}\left\langle s_{j}, s_{j}\right\rangle\right) \\
& =(\mathrm{r}-\operatorname{Ind}[X]) \sum_{i} \tau\left(\left\langle u_{i}, u_{i}\right\rangle_{A}\right) \\
& =(\mathrm{r}-\operatorname{Ind}[X]) \hat{\tau}\left(\left[\mathcal{E}_{A}\right]\right) \\
& =V\left(\hat{\tau}\left[\mathcal{E}_{A}\right]\right) .
\end{aligned}
$$

By using this proposition, we have the following theorem.

Theorem 5.9. For a Hilbert $C^{*}$-bimodule $X={ }_{A} X_{A}$ of finite type, the following identity holds:

$$
d(X)=d_{\tau}(\varphi(X)) .
$$

Moreover, $d$ and $\phi$ conserve sum and product operations. On the other hand, $d_{\tau}$ conserves only sum operation.

Proof. Using Proposition 5.8,

$$
d_{\tau}\left(\varphi\left({ }_{A} X_{A}\right)\right)=d_{\tau}\left(\left[{ }_{A} X_{A}\right]\right)=\hat{\tau}\left(\gamma_{[X]}([1])\right)=V \hat{\tau}(1)=d(X) 1 .
$$


Corollary 5.10. In the same situation, for a Hilbert $A-A$ bimodule $X$ of finite type which is compatible with $\tau$, its statistical dimension $d(X)$ depends only on its equivalence class in $K K(A, A)$.

We let $\mathcal{F}$ be the subring in $K K(A, A)$ generated by the image of $\varphi$, i.e.

$$
\begin{array}{r}
\mathcal{F}=\left\{\left[{ }_{A} X_{A}\right]-\left[{ }_{A} Y_{A}\right] \in K K(A, A): X, Y \text { minimal normalized Hilbert } A \text { - } A\right. \\
\text { bimodules of finite type which are } \tau \text { compatible }\} .
\end{array}
$$

Corollary 5.11. In the same situation $d_{\tau}$ is multiplicative on $\mathcal{F}$. i.e. $d_{\tau}(\alpha \otimes \beta)=$ $d_{\tau}(\alpha) d_{\tau}(\beta) . d_{\tau}$ is a character on $\mathcal{F}$.

Proof. This is a direct consequence of the multiplicativity of $d$.

Example. Let $e_{1}, e_{2}, e_{3}, \ldots$ be Jones projections such that

$$
e_{i} e_{i \pm 1} e_{i}=\left(4 \cos ^{2} \frac{\pi}{5}\right)^{-1} e_{i}
$$

We have $4 \cos ^{2} \frac{\pi}{5}=\frac{3+\sqrt{5}}{2}=\phi^{2}$, where $\phi=\frac{1+\sqrt{5}}{2}$ is the golden number. Put $A=$ $\mathrm{C}^{*}\left\{1, e_{2}, e_{3}, \ldots\right\}, B=\mathrm{C}^{*}\left\{1, e_{1}, e_{2}, e_{3}, \ldots\right\}$. Then $A \subset B$, and $K_{0}(A)$ is isomorphic to $\mathbf{Z} \oplus \phi \mathbf{Z}$, and $K_{1}(A)$ is zero. $\hat{\tau}: K_{0}(A) \rightarrow \mathbf{R}$ is given by $\hat{\tau}(m, n)=m+\phi n$. Then

$$
K K(A, A) \simeq M_{2}(\mathbf{Z})=\left\{\left(\begin{array}{ll}
a & b \\
c & d
\end{array}\right): a, b, c, d \in \mathbf{Z}\right\} .
$$

For $\gamma_{\alpha}=\left(\begin{array}{ll}a & b \\ c & d\end{array}\right) \in \operatorname{Hom}\left(K_{0}(A), K_{0}(A)\right)$, we have

$$
d_{\tau}(\alpha)=\hat{\tau}\left(\gamma_{\alpha}[1]\right)=\hat{\tau}\left(\left(\begin{array}{ll}
a & b \\
c & d
\end{array}\right)\left(\begin{array}{l}
1 \\
0
\end{array}\right)\right)=\hat{\tau}\left(\begin{array}{l}
a \\
c
\end{array}\right)=a+\phi c .
$$

By Example (3.3.14) in [47, $\varphi\left(\left[{ }_{A} B_{A}\right]\right) \in K K(A, A)$ is identified with the matrix

$$
S=\left[\begin{array}{ll}
1 & 1 \\
1 & 2
\end{array}\right]
$$

and $\varphi\left(\left[{ }_{A} A_{A}\right]\right)$ is identified with the identity matrix $I$. Then

$$
\left[\begin{array}{cc}
a & b \\
b & a+b
\end{array}\right]=(a-b) I+b S \in \mathcal{F}
$$

Conversely, we take

$$
\left[\begin{array}{ll}
a & b \\
c & d
\end{array}\right] \in \mathcal{F}
$$

Since $d_{\tau}$ is multiplicative,

$$
d_{\tau}\left(\left[\begin{array}{ll}
a & b \\
c & d
\end{array}\right]\left[\begin{array}{ll}
1 & 1 \\
1 & 2
\end{array}\right]\right)=d_{\tau}\left(\left[\begin{array}{ll}
a & b \\
c & d
\end{array}\right]\right) d_{\tau}\left(\left[\begin{array}{ll}
1 & 1 \\
1 & 2
\end{array}\right]\right) .
$$

Considering $\phi^{2}=\phi+1$, we have $a+b=a+c, c+d=a+2 c$. We conclude that $b=c$ and $d=a+b$.

In this case

$$
\mathcal{F}=\left\{\left(\begin{array}{cc}
a & b \\
b & a+b
\end{array}\right) \in M_{2}(\mathbf{Z}): a, b= \pm 0,1,2, \ldots\right\} .
$$


Finally, we consider a Goldman type theorem for $\mathrm{C}^{*}$-algebras. If $N \subset M$ is the inclusion of $I I_{1}$-factors and if $[M: N]=2$, then there exists an outer action $\alpha$ of $\mathbf{Z}_{2}$ on $N$ such that $M$ is the crossed product $N \rtimes_{\alpha} \mathbf{Z}_{2}$. In the $\mathrm{C}^{*}$-setting, a Goldman type theorem does not hold in the original sense. The following shows that there is a K-theoretic obstruction here.

Theorem 5.12. Let $\mathrm{O}_{2}$ be the Cuntz algebra of 2-generators. There exists an outer action $\alpha$ of $\mathbf{Z}_{2}$ on $\mathrm{O}_{2}$ such that there exists no action of $\mathbf{Z}_{2}$ on $\mathrm{O}_{2}^{\alpha}$ such that $\mathrm{O}_{2}$ is isomorphic to the $C^{*}$-crossed product of $\mathrm{O}_{2}^{\alpha}$ by this action.

Proof. Let $S_{1}$ and $S_{2}$ be a generating pair of $O_{2}$. We define an automorphism $\alpha$ of $\mathrm{O}_{2}$ by $\alpha\left(S_{i}\right)=-S_{i}$. The fixed point algebra is generated by the family $\left\{S_{1}{ }^{2}, S_{2}{ }^{2}, S_{1} S_{2}, S_{2} S_{1}\right\}$. These generators also satisfy the generating condition of $\mathrm{O}_{4}([13])$.

Define a conditional expectation $E: O_{2} \rightarrow O_{2}^{\alpha}$ by $E(x)=\frac{1}{2}(x+\alpha(x))$ for $x \in O_{2}$. Then $\operatorname{Ind}[E]=2$ by the remark after 3.3 in [47].

We assume that $O_{4} \rtimes_{\beta} \mathbf{Z}_{2} \simeq O_{2}$ by some action $\beta$ of $\mathbf{Z}_{2}$. Then by TakesakiTakai duality, $\mathrm{O}_{4} \otimes M_{2}(\mathbf{C}) \simeq \mathrm{O}_{2} \rtimes_{\hat{\beta}} \mathbf{Z}_{2}$. On the other hand, $K_{0}\left(O_{2}\right) \simeq\{0\}$ and $K_{0}\left(O_{4}\right) \simeq\{0,1,2\}$. The identity of $O_{2}$ is zero as a $K_{0}$-element, and is mapped into the identity of $\mathrm{O}_{4} \otimes M_{2}(\mathbf{C})$, which is not zero in $K_{0}\left(O_{4}\right)$. From these, we have a contradiction.

On the other hand, for a dual inclusion, a Goldman type theorem holds in the sense of realizing it as a crossed product by $\mathbf{Z}_{2}$.

Theorem 5.13. Let $A \subset B$ be a unital inclusion of $C^{*}$-algebras and $E$ a conditional expectation from $B$ to $A$ such that $\operatorname{Ind}[E]=2 \cdot I$. Let $C$ be the $C^{*}$-basis extension $\mathrm{C}^{*}\left\langle B, e_{A}\right\rangle$. Then there exists an action $\alpha$ of $\mathbf{Z}_{2}$ on $B$ such that $A$ is the fixed point algebra $B^{\alpha}$ and $C$ is isomorphic to the crossed product $B \rtimes_{\alpha} \mathbf{Z}_{2}$.

Proof. Let $e_{A}$ be the orthogonal projection from $B$ to $A$. There exists a conditional expectation $F$ dual to $E$ such that

$$
F\left(x e_{A} y\right)=(\operatorname{Ind}[E])^{-1} x y=\frac{1}{2} x y .
$$

Since $\operatorname{Ind}[E]=2 \cdot I \in Z(A)$, we have Index $E=\operatorname{Index} F=2$ by Proposition 2.3.4 in [47]. Let $D=\mathrm{C}^{*}\left\langle C, e_{B}\right\rangle$ be the $\mathrm{C}^{*}$-basis construction. Similarly we have a conditional expectation $G: D \rightarrow C$ dual to $F$ such that $G\left(e_{B}\right)=\frac{1}{2} I$ and $G\left(e_{A}\right)=e_{A}$ and $\operatorname{Ind}[G]=2 \cdot I$. We define two unitary elements $u \in C$ and $v \in D$ by $u=2 e_{A}-1$ and $v=2 e_{B}-1$. We show that $u v=-v u$. We define $p$ as follows: $p=2\left(e_{A}+e_{B}-e_{A} e_{B}-e_{B} e_{A}\right)$. Then $p$ is a selfadjoint projection by $e_{B} e_{A} e_{B}=\frac{1}{2} e_{B}$ and $e_{A} e_{B} e_{A}=\frac{1}{2} e_{A}$. Since

$$
G(p)=2\left(e_{A}+\frac{1}{2} I-\frac{1}{2} e_{A}-\frac{1}{2} e_{A}\right)=I,
$$

$p$ must be $I$ because $G$ is faithful by Remark (4) after Definition 1.2.2 in [47]. Substituting $e_{A}=\frac{1}{2}(u+I), e_{B}=\frac{1}{2}(v+I)$ in the equation

$$
p=2\left(e_{A}+e_{B}-e_{A} e_{B}-e_{B} e_{A}\right)=I
$$

we have $u v=-v u$.

Then for all $b \in B, u b u^{*}$ commutes with $v=2 e_{B}-1$. Then $u b u^{*} \in B$ by Lemma 2.1.1 in [47]. Put $\alpha=\operatorname{ad} u$. Then $\alpha$ is an automorphism of $B$. 
Since $b \in B^{\alpha}$ if and only if $b$ commutes with $u=2 e_{A}-1$, we have $B^{\alpha}=A$. Since $F(u)=0$, the map from $B \rtimes_{\alpha} \mathbf{Z}_{2}$ to $C=B e_{A} B=B+B u$ defined as $x+y u$ is well defined and is an isomorphim.

We are informed that M. Izumi has obtained the characterization of depth 2 inclusion by fixed point algebras by Kac algebra for simple $\mathrm{C}^{*}$-algebras. He also characterized group-subgroup inclusion for simple $\mathrm{C}^{*}$-algebras.

\section{REFERENCES}

[1] B.Blackadar, K-theory for operator algebras, MSRI Publ., vol. 5, Springer-Verlag, 1986. MR 88g:46082

[2] L.G.Brown, P.Green and M.A.Rieffel, Stable isomorphism and strong Morita equivalence of $C^{*}$-algebras, Pacific J. Math. 71 (1977), 349-363. MR 57:3866

[3] L.G.Brown,J.A.Mingo and N.T.Shen, Quasi-multipliers and embeddings of Hilbert $C^{*}$ bimodules, Canad. J. Math. 46 (1994), 1150-1174. MR 95k:46091

[4] M.Choda and H.Kosaki, Strongly outer actions for an inclusion of factors, J. Funct. Anal. 122 (1994), 315-332. MR 96b:46085

[5] A.Connes, Noncommutative Geometry, Academic Press (1994). MR 95j:46063

[6] V.Deaconu, $C^{*}$-Algebras associated with Branched Coverings, preprint.

[7] V.Deaconu, A path model for circle algebras, J. Operator Theory 34 (1995), 57-89. MR 96m:46113

[8] Y.Denizeau and J.-F.Havet, Correspondences d'indice fini I, J. Operator Theory 32 (1994), 111-156. MR 96e:46081

[9] Y.Denizeau and J.-F.Havet, Correspondences d'indice fini II, Topics in Operator Theory, Operator Algebras and Applications, Rom. Acad. Bucharest (1995), 49-79. [MR 97i:46110]

[10] S.Doplicher,R.Haag and J.E.Roberts, Local observables and particle statistics I, Commun.Math.Phys. 23 (1971), 199-230. MR 45:6316

[11] S.Doplicher,R.Haag and J.E.Roberts, Local observables and particle statistics II, Commun.Math.Phys. 35 (1974), 49-85. MR 48:13060

[12] S.Doplicher and J.E.Roberts, A new duality theory for compact groups, Invent.Math. 98 (1989), 157-218. MR 90k:22005

[13] M.Enomoto, M.Fujii, H.Takehana and Y.Watatani, Automorphisms on Cuntz Algebras, II, Math.Japonica 24 (1979), 463-468. MR 81i:46086b

[14] D.Evans and Y.Kawahigashi, On Ocneanu's theory of asymptotic inclusions for subfactors, topological quantum field theories and quantum double, Internat. J. Math. 6 (1995), 205-228. MR 96d: 46080

[15] R.Exel, A Fredholm operator approach to Morita equivalence, K-Theory 7 (1993), 285-308. MR 94h:46107

[16] F.M.Goodman, P.de la Harpe and V.F.R. Jones, Coxeter Graphs and Towers of Algebras, MSRI Publications, vol. 14, Springer-Verlag, 1989. MR 91c: 46082

[17] P.Ghez, R.Lima and J.E.Roberts, $W^{*}$-categories, Pac.J.Math. 120(1985) 79-109. MR 87g:46091

[18] J.-F.Havet, Esperence conditionelle minimele, J.Operator Theory 24 (1991), 33-55. MR 92a:46073

[19] F.Hiai, Minimizing indices of conditional expectation onto a subfactor, Publ.RIMS 24 (1988), 673-678. MR 90a:46157

[20] M.Izumi, Application of fusion rules to classification of subfactors, Publ.RIMS.Kyoto Univ. 27 (1991), 953-994. MR 93b:46121]

[21] M.Izumi, Goldman's type theorem for index 3, Publ.RIMS.Kyoto.Univ. 28 (1992), 833-843. MR 94m:46093

[22] M.Izumi, Subalgebras of infinite $C^{*}$-algebras with finite Watatani Indices I. Cuntz algebras, Comm.Math.Phys. 155 (1995), 157-182. [MR 94e:46104]

[23] V.F.R.Jones, Index for subfactors, Inv. Math. 72 (1983), 1-25. MR 84d:46097

[24] L.Kadison, On split, separable subalgebras with counitality condition, Hokkaido Math. J. 24 (1995), 527-549. MR 98b:46081

[25] C.Kassel, Quantum Groups, Graduate Texts in Math., vol. 155, Springer-Verlag, 1995. MR 96e:17041 
[26] G.G.Kasparov, Hilbert $C^{*}$-module: Theorem of Steinspring and Voiculescu, J.Operator Theory 4 (1980), 133-150. MR 82b:46074

[27] G.G.Kasparov, The operator K-functor and extensions of $C^{*}$-algebras, Math.USSR-Izv 16 (1981), 513-572. MR 81m:58075

[28] S.Kawakami and Y Watatani, The multiplicativity of the minimal index of simple $C^{*}$ algebras, Proc. Amer. Math. Soc. 123 (1995), 2809-2813. MR 95k:46093

[29] M.Khoshkam, Hilbert $C^{*}$-modules and conditional expectations on crossed products, J. Austral. Math. Soc. (Series A) 61 (1996), 106-118. MR 97i:46100

[30] K.Kodaka, Endomorphisms of certain irrational rotation $C^{*}$-algebras, Illinois J. Math. 36 (1992), 643-658. MR 94b:46086

[31] H.Kosaki, Automorphisms in the irreducible decomposition of sectors, Quantum and Noncommutative Analysis, Kluwer Academic (1993), 305-316. MR 95e:46073

[32] H.Kosaki and R.Longo, A remark on the minimal index of subfactors, J.Funct.Anal. 107 (1992), 458-470. MR 93i:46108

[33] H.Kosaki and S.Yamagami, Irreducible bimodules associated with crossed product algebras, Internat. J. Math. 3 (1992), 661-676. MR 94f:46087

[34] R.Longo, Index of subfactors and statistics of quantum fields I, Commun.Math.Phys. 126 (1989), 245-155. MR 91c:46097

[35] R.Longo, Index of subfactors and statistics of quantum fields II, Commun.Math.Phys. 130 (1990), 285-309. MR 91j:46083

[36] R.Longo, Minimal index and braided subfactors, J.Funct.Anal. 107 (1992), 458-470. MR 93i:46109

[37] A.Ocneanu, Quantized groups, string algebras and Galois theory for algebras, Operator algebras and applications,London Math.Soc. Lect.Notes.Series 2 (1988), 119-172. MR 91k:46068

[38] A.Ocneanu, Quantized symmetry, differential geometry of finite graphs and classification of subfactors (Note recorded by Y.Kawahigashi), University of Tokyo Seminor Notes 45 (1991).

[39] W.Paschke, Inner product modules over B*-algebras, Trans. AMS. 182 (1973), 443-468. MR 50:8087

[40] C.Peligrad and W.Szymanski, Saturated Actions of Finite Dimensional Hopf *-algebras on $C^{*}$-Algebras, Math. Scand. 75 (1994), 217-239. MR 96b:46097

[41] M.Pimsner and S.Popa, Entropy and index for subfactors, Ann.Sc.Ec.Norm.Sup. 19 (1985), 57-106. MR 87m:46120

[42] S.Popa, Correspondences, preprint.

[43] M.A.Rieffel, Induced representations of $C^{*}$-algebras, Adv.Math. 13 (1974), 176-257. MR 50:5489

[44] M.A.Rieffel, Morita equivalence for $C^{*}$-algebras and $W^{*}$-algebras, J.Pure Appl. Algebra 5 (1974), 51-96. MR 51:3912

[45] M.A.Rieffel, Morita equivalence for operator algebras, Proc Symp. in Pure Math. 38, 285-298. MR 84k:46045

[46] V.S.Sunder, $I I_{1}$-factor, their bimodules and hypergroups, Trans.Amer.Math.Soc. 330 (1992), 227-256. MR 92f:46076

[47] Y.Watatani, Index for $C^{*}$-subalgebras, Memoir AMS 424 (1990). MR 90i:46104

[48] S.Yamagami, A note on Ocneanu's approach to Jones index theory, Intnat.J.Math. 4 (1993), 859-871. MR 95f:46114

[49] S.Yamagami, On unitary representation theory of compact quantum groups, Comm. Math.Phys. 167 (1995), 509-529. MR 96a:46114

[50] S.Yamagami, Frobenius reciprocity in tensor categories, preprint (1993).

[51] S.Yamagami, Modular theory for bimodules, J.Funct.Anal 125 (1994), 327-357. MR 96h:46100

Department of Environmental and Mathematical Sciences, Okayama Unniversity, TSUShima, OKaYAma 700, JaPan

E-mail address: kajiwara@math.ems.okayama-u.ac.jp

Graduate School of Mathematics, Kyushu University, Ropponmatsu, Fukuoka, 810 JAPAN

E-mail address: watatani@math.kyushu-u.ac.jp 